\title{
Analysis of the ${ }^{12} C\left(e, e^{\prime} p d\right)$ Reaction at High Energy Transfer
}

Neil Graham Thompson

Presented as a Thesis for the Degree of Doctor of Philosophy

\author{
Nuclear Physics Group \\ Department of Physics and Astronomy \\ University of Glasgow
}

Copyright N.G. Thompson 2011 


\section{Abstract}

The Jefferson Lab Hall A experiment E01015 measured the differential cross sections of the ${ }^{12} C\left(e, e^{\prime} p d\right)$ reaction with low yields for kinematics of $\omega=0.865$ $\mathrm{GeV}, Q^{2}=2(\mathrm{GeV} / \mathrm{c})^{2}, x_{B}=1.2$ at three kinematic settings of ${ }^{12} \mathrm{C}\left(e, e^{\prime} p\right)$ missing momentum of 300, 400 and $500 \mathrm{MeV} / \mathrm{c}$. The main objective of the experiment was to measure the ${ }^{12} C\left(e, e^{\prime} p\right)$ cross section and the cross section ratios of ${ }^{12} C\left(e, e^{\prime} p p\right)$ and ${ }^{12} C\left(e, e^{\prime} p n\right)$ to investigate Short Range Correlations. The ${ }^{12} C\left(e, e^{\prime} p d\right)$ reaction was investigated to find out what its magnitude of cross section. The very low yields and differential cross sections of ${ }^{12} C\left(e, e^{\prime} p d\right)$ reaction measured at the three different kinematic settings suggests that the contribution of many of the different reaction mechanisms, including three nucleon forces, may be suppressed at the kinematics of this experiment. 


\section{Declaration}

The data presented in this thesis were obtained as part of the SRC collaboration at the U.S. Department of Energy's Jefferson Lab in Virginia and the University of

Glasgow's Nuclear Physics Group. I participated in the preparation and execution of the experiment. The analysis of the experimental data is my own work and the thesis was composed by myself.

\section{Neil G. Thompson}

March 2011 


\section{Contents}

1 Introduction $\quad 1$

1.1 Previous Measurements . . . . . . . . . . . . . . . . . . 3

1.1.1 Measurement of 2- and 3-Nucleon Short Range Correlation Probabilities in Nuclei . . . . . . . . . . . . . . . . 4

1.1.2 Study of Three-Nucleon Mechanisms in the Photodisintegration of ${ }^{3} \mathrm{He} \ldots \ldots \ldots \ldots \ldots$

1.1.3 Two-body photodisintegration of ${ }^{3} \mathrm{He}$ between 200 and 800 $\mathrm{MeV} \ldots \ldots \ldots \ldots \ldots \ldots$

1.1.4 Three-nucleon mechanisms in photoreactions . . . . . . . 8

1.1.5 Dependence of the ${ }^{12} C(\vec{\gamma}, p d)$ Reaction on Photon Linear Polarisation .................... 14

1.1.6 The $(\gamma, p d)$ reaction in ${ }^{12} C \ldots \ldots \ldots \ldots$

1.1.7 Investigation of the Exclusive ${ }^{3} \mathrm{He}\left(e, e^{\prime} p n\right)^{1} \mathrm{H}$ Reaction . . 20

2 Experiment E01015 24

2.1 Thomas Jefferson National Accelerator Facility . . . . . . . . . . 25

2.2 Experimental Hall A . . . . . . . . . . . . . . . . . . . . . . 27

2.2.1 Electron Beam Monitors . . . . . . . . . . . . . . . 28

2.2 .2 Target System . . . . . . . . . . . . . . . . . . . . . . . . . 30

2.2.3 High Resolution Spectrometer . . . . . . . . . . . . . . 31

2.2.4 HRS Focal Plane Detectors . . . . . . . . . . . . . . . . . 32

2.2.5 BigBite Spectrometer . . . . . . . . . . . . . . . 36

2.2 .6 Neutron Detector . . . . . . . . . . . . . . . . 38

2.3 Data Acquisition . . . . . . . . . . . . . . . . . . . . . 40

2.3.1 Trigger Scheme . . . . . . . . . . . . . . . . . 40

2.3.2 Electronics. . . . . . . . . . . . . . . . . . 42 
3 Data Analysis: Event Selection $\quad 44$

3.1 Overview . . . . . . . . . . . . . . . . . . . . . . . 44

3.2 Electron Analysis . . . . . . . . . . . . . . . . . . . . . . . . . . 45

3.2.1 VDC Track Reconstruction . . . . . . . . . . . . . . 45

3.2.2 Target Coordinate Reconstruction . . . . . . . . . . . 47

3.2 .3 Electron Selection . . . . . . . . . . . . . . . 50

3.3 Right HRS Analysis . . . . . . . . . . . . . . . . . 51

3.3.1 ${ }^{12} C\left(e, e^{\prime} p\right)$ Kinematic Cuts . . . . . . . . . . . . 54

3.4 BigBite Analysis . . . . . . . . . . . . . . 56

3.4.1 Bar Hit Position Calibration . . . . . . . . . . . . 56

3.4.2 ADC Calibration . . . . . . . . . . . . . . 58

3.4.3 TOF Calibration ................. . . 61

3.4.4 Tracking and Momentum Calculation . . . . . . . . . . . 62

3.4.5 Geant4 Simulation . . . . . . . . . . . . . . 66

3.4.6 BigBite Event Selection . . . . . . . . . . . . . . . 69

3.4.7 Yield . . . . . . . . . . . . . . . . . . . 75

4 Simulation and Acceptance Correction $\quad 83$

4.1 (e,e'pd) Simulation . . . . . . . . . . . . . . . . 83

4.1.1 Event Generator . . . . . . . . . . . . . . . . . . 83

4.1.2 Extraction of Simulated Data . . . . . . . . . . . . . 84

4.1.3 Comparison of Generated, Simulated and Experimental Data 86

4.1.4 Acceptance Correction . . . . . . . . . . . . . . . 88

4.2 Luminosity . . . . . . . . . . . . . . . . . . . . . . . . . . . . . 89

$\begin{array}{llr}5 & \text { Results and Conclusion } & 93\end{array}$ 


\section{List of Figures}

1.1 Weighted cross section ratios of (a) ${ }^{4} \mathrm{He}$, (b) ${ }^{12} \mathrm{C}$ and (c) ${ }^{56} \mathrm{Fe}$ to ${ }^{3} \mathrm{He}$ as a function of $x_{B}$ for $Q^{2}>1.4 \mathrm{GeV}^{2}$. The horizontal dashed lines indicate the $N N$ and $3 N$ scaling regions used to calculate the per-nucleon probabilities for 2 - and $3 N$ SRCs in nucleus $A$ relative to ${ }^{3} H$. . . . . . . . . . . . . . . . . . . . . . . . . .

1.2 (i) The total cross section of the $\gamma^{3} H e \rightarrow p p n$ channel integrated over the detector acceptance (black squares) is compared to the theoretical predictions of Laget. (ii) The total cross section for $3 N$ photoabsorption estimated from the experimental data compared to results obtained by the TAGX experiment, which measured the same reaction channel for a photon energy range from $200-500$ $\mathrm{MeV}$ using the spectrometer TAGX, which has a solid angle for protons of $\pi s r$. Discrepancies in the comparison of results are probably due to the Laget model used in this experiment and its ability to calculate the $2 N$ contribution in good agreement with previous data. . . . . . . . . . . . . . . . . . .

1.3 (i) Triangular Dalitz plot at $400 \mathrm{MeV}$ : the three axes are the c.m. kinetic energies of the proton $_{1}$, proton $_{2}$, and neutron normalised to the total c.m. energy. Events in the region between the 2 parallel lines were selected for the study of the $3 N$ mechanisms. (ii) Distribution, at $300 \mathrm{MeV}$, of the invariant mass of the object $\mathrm{X}$ which is exchanged between the proton and the neutron-pair.

1.4 The excitation curves at proton c.m. angles of $60^{\circ}$ (a) and $90^{\circ}$ (b) obtained in this experiment compared with previous measurements. 8 
1.5 Angular distributions at photon energies of 267, 326, 420, 450, 500, 550 and $650 \mathrm{MeV}$, obtained in this experiment compared to previously published data (up to $550 \mathrm{MeV}$ ) and to theoretical calculations of Laget [1]. The continuous curve gives the contribution of one and two body mechanisms while the dashed curve also includes the contribution of three body processes. . . . . . . . . .

1.6 Three-body missing energy distributions for the ${ }^{12} C(\gamma, p p n)$ reaction. The shaded histograms show the VM predictions for the different reaction mechanisms indicated by the key on the figure. The solid line shows the total cross section without the reduction of the $N \pi+$ ABS prediction. . . . . . . . . . . . .

1.7 Measured and predicted $\left(M_{X} / m_{\pi}\right)^{2}$ distributions for the ${ }^{12} C(\gamma, p p n)$ reaction at missing energy of $\leq 100 \mathrm{MeV}$ and $E_{\gamma}=150-500$ $\mathrm{MeV}$. Bottom left panel shows the Valencia model predictions of $\left(M_{X} / m_{\pi}\right)^{2}$ for the neutron versus the forward proton. The projections onto the $x$ and $y$ axes (shaded histograms) are compared with the experimental data in the top left and bottom right panels. The solid line shows the total cross section without the reduction of the $N \pi+$ ABS prediction. . . . . . . . . . . . . .

1.8 The ${ }^{12} C(\gamma, p p n)$ cross section as a function of photon energy presented for three missing energy regions indicated in the figure. The lower figures have the additional cut $\left(M_{X} / m_{\pi}\right)^{2} \leq-1.5$ for the neutron and the most forward-angle proton. The total prediction of the (modified) Valencia model is shown by the thick solid line and the separate contributions from the $2 N+$ FSI, $3 N$ (with or without FSI), $N \pi+\mathrm{ABS}$, and $N N \pi / N \pi+$ EMIT processes are shown by the short dash, long dash, dotted and dot-dashed lines respectively. The thin solid line shows the total VM without reducing the $N \pi+$ ABS strength. . . . . . . . . . . . 
1.9 (i) Cross section for the $(\gamma, p d),(\gamma, p p)$ and $(\gamma, p n)$ reactions versus missing energy. The data presented for $E_{\gamma}$ bins of (a) 170$220 \mathrm{MeV}$, (b) 220-280 MeV and (c) 280-350 MeV. (ii) (a) The ${ }^{12} C(\vec{\gamma}, p d),{ }^{12} C(\vec{\gamma}, p n)$ and ${ }^{12} C(\vec{\gamma}, p p)$ photon asymmetry integrated over $\theta_{p}=51^{\circ}-120^{\circ}$ for the missing energy cuts indicated in (i) (a), (b), (c). The errors are statistical only. The experimental results [2] for ${ }^{3} \mathrm{He}(\vec{\gamma}, p d)$ at $\theta_{p}=90^{\circ}$ and $110^{\circ}$ and the Faddeev predictions [3] at $\theta_{p}=110^{\circ}$ are also shown. The red (solid) and green (dot-dash) lines give the asymmetry at $\theta_{\pi}=55^{\circ}$ from the MAID [4] calculations for $p\left(\vec{\gamma}, \pi^{0}\right) p$ and $p\left(\vec{\gamma}, \pi^{+}\right) n$ respectively. The corresponding predictions without including $\Delta(1232)$ are shown by the red (dashed) and green (dotted) lines. (b) Comparison of the ${ }^{12} C(\vec{\gamma}, p d),{ }^{12} C(\vec{\gamma}, p n)$ and ${ }^{12} C(\vec{\gamma}, p p)$ asymmetry at higher missing energy. . . . . . . . . . . . . . .

1.10 Missing energy spectra for the ${ }^{12} C(\gamma, p d)$ reaction for backward (thick), central (thin), and forward (dashed) proton angles. . . . .

1.11 Photon energy dependence of the ${ }^{12} C(\gamma, p d)$ reaction for $E_{m}<44$ $\mathrm{MeV}$ (left scale) compared to that for the ${ }^{3} \mathrm{He}(\gamma, p d)$ reaction [5] (right scale) and the ${ }^{3} \mathrm{He}(\gamma, p p) n$ reaction [6] (total cross section, right scale times 50). The ${ }^{12} \mathrm{C}$ data are expressed in units of $n b / s r^{2}$, the ${ }^{3} \mathrm{He}(\gamma, p d)$ data are in $n b / s r$, and the ${ }^{3} \mathrm{He}(\gamma, p p) n$ data are

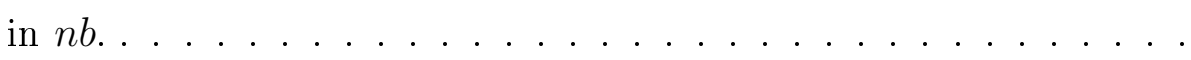

1.12 Recoil momentum distributions for the ${ }^{12} C(\gamma, p d)$ reaction for (i) $E_{m}<44 \mathrm{MeV}$ and (ii) $E_{m} 44-70 \mathrm{MeV}$. The curves show the distributions predicted by the simple model described in the text. FWD, CEN, and BCK refer to the proton detector angles of $22.7^{\circ}-$ $101.1^{\circ}, 51.3^{\circ}-128.6^{\circ}$, and $79.0^{\circ}-156.7^{\circ}$ respectively. . . . . . .

1.13 The ${ }^{3} \mathrm{He}\left(e, e^{\prime} p n\right)$ reaction cross section averaged out over the experimental acceptance, as a function of missing momentum for the A1 kinematic setting. The solid red (dotted green) curve shows the theoretical cross section calculated using a one-body hadronic current operator and the AV18 (BB) $N N$ potential. The dashed blue line results from the AV18 potential when MECs are also included. 
1.14 The ${ }^{3} \mathrm{He}\left(e, e^{\prime} p n\right)$ reaction cross section shown as a function of $q$ for $50 \leq p_{m} \leq 100 \mathrm{MeV} / \mathrm{c}$ and $235 \leq \omega \leq 265 \mathrm{MeV}$. Results from different kinematic settings are shown by the types of markers for the data points. The line convention is the same as in fig. 1.13. The theoretical cross section was calculated at the points indicated by the black circles. . . . . . . . . . . . . . . . . . . . 23

2.1 Schematic of the experimental layout for E01015 in Hall A at JLab. 25

2.2 Schematic of CEBAF. . . . . . . . . . . . . . . . . 27

2.3 Schematic of Hall A. . . . . . . . . . . . . . . . . . . . . . 28

2.4 Picture of target ladder. Liquid deuterium and hydrogen cells are at the top and some carbon foil targets are at the bottom of the target. . . . . . . . . . . . . . . . . 30

2.5 Side view of HRS showing the layout of magnet field boundaries. . 31

2.6 Diagram of left and right HRS detector packages. . . . . . . . . 33

2.7 The position of the VDCs relative to each other, and to the nominal central trajectory. . . . . . . . . . . . . . . . . . . . 34

2.8 Diagram of the left HRS shower counter. Particles enter form the bottom of the figure.

2.9 CAD drawing of the BigBite magnet and its detector package consisting of the auxiliary and trigger scintillator planes. . . . . . . .

2.10 Diagram of BigBite produced from Geant 4 simulation $[7,8]$. It shows three particle tracks of different momentum and out-of-plane angle including the central ray of $500 \mathrm{MeV} / \mathrm{c}$. . . . . . . . . . 38

2.11 Scintillator and veto bar configuration of the Neutron Detector. . 40

2.12 Data acquisition packages of BigBite and the Neutron Detector. Picture courtesy of Peter Monaghan [9]. . . . . . . . . . . . . .

3.1 A typical cluster of hits in a VDC wire plane. $C_{i}$ denotes the $i$ th wire cell, and $d_{i}$ represents the perpendicular (effective) drift distance within that cell. The $i$ th cell is defined as the region within which ions drift to the $i$ th wire. By symmetry, the cell boundaries lie halfway between wires. The wire spacing is 4.24 $\mathrm{mm}$. The separation between cathode high-voltage and wire planes is approximately $13 \mathrm{~mm}$. The HV planes are held at a potential of approximately $-4 \mathrm{kV}$, while the wires are effectively grounded. Figure taken from [10]. . . . . . . . . . . . . . . . . . . . . . . . . 46 
3.2 Coordinates in the TRANSPORT convention. $z$ is defined by the central or reference trajectory. $x$ and $y$ are perpendicular to $z$, with $x$ in the dispersive plane. . . . . . . . . . .

3.3 Target system electron projection parameters $\delta, \phi_{t g t}, y_{t g t}$ and $\theta_{t g t}$ after optimisation of the HRS optics tensors, using production data taken at the CK1 kinematics $\left({ }^{12} C\left(e, e^{\prime} p\right)\right.$ missing momentum of $300 \mathrm{MeV} / \mathrm{c}) . \ldots \ldots \ldots \ldots$

3.4 These histograms show the process of selecting electrons and rejecting pions from the tracks in the left HRS. Top-Left: The pre-shower energy deposited versus the shower energy deposited, with the electrons contained in the large distribution in the centre and the pions encircled by a 2D cut in the bottom left. The small upper distribution is due to a small calibration error of the pre-shower. TopRight: Histogram showing the addition of the energy deposited in the pre-shower and shower counter. The events in the large peak are identified as electrons and the small peak (around channel 500) contains pions identified by the graphical cut. Bottom-Left: Plot showing the light output from the Cherenkov detector. Only the electrons deposit any significant amount of energy. Pions correspond to the large spike around zero. To remove these pions, only events where the Cherenkov ADC was above 50 channels were selected. Bottom-Right: Cherenkov energy versus the total shower counter energy. . . . . . . . . . . . . . . . .

3.5 Top: The $\beta$ spectrum of the particles in the right HRS. The large peak to the right contains protons $(\beta \sim 0.85)$ and the smaller peak represent deuterons $\beta \sim 0.63$. Bottom: The coincidence time versus $\beta$. The coincidence time of the protons is around $100 \mathrm{~ns}$ and the deuterons are around $140 \mathrm{~ns} \ldots \ldots \ldots$. . . . . .

3.6 Top: $\beta$ versus deposited energy in the $\mathrm{S} 2$ scintillator plane. The majority of the pions are within the blue $2 \mathrm{D}$ cut. Bottom: $\beta$ versus the momentum of the track. Most of the pions have been removed from this plot and the red $2 \mathrm{D}$ cut selects the protons in the the main distribution and the green $2 \mathrm{D}$ cut selects the deuterons. . .

3.7 Top: The coincidence time (log scale) of the (e,e'p) events is selected within the red cut lines and the regions bordered by the green lines contain the events selected for background estimates. Bottom: The coincidence time plot for (e,e'd) events. . . . . . . . 
3.8 Top-Left: Missing energy of ${ }^{12} C\left(e, e^{\prime} p\right)$ reaction. The pronounced ground state peak at about $30 \mathrm{MeV}$ is removed by the cut (red line) at $32 \mathrm{MeV}$. In the continuum there are two broad peaks at $\sim 130$ and $\sim 240 \mathrm{MeV}$. Top-Right: Transferred energy $\omega$ versus missing energy. Bottom-Left: Recoil nucleus angle versus $\omega$. Bottom-Right: Recoil nucleus angle versus missing energy. All histograms are from the first kinematic setting of ${ }^{12} C\left(e, e^{\prime} p\right)$ missing momentum of $300 \mathrm{MeV} / \mathrm{c}$. The pronounced ground state in missing energy spectrum peak at about $30 \mathrm{MeV}$ is removed by the cut (red line) at $32 \mathrm{MeV}$. In the missing energy continuum there are two broad peaks at $\sim 130$ and $\sim 240 \mathrm{MeV}$. The $130 \mathrm{MeV}$ peak is at lower transferred energy and higher backward angles than the $240 \mathrm{MeV}$ peak, which is due to the $\Delta$ excitation. A cut above $77^{\circ}$ was used to cut the contributing $\Delta$ excitations. . . . . . . . .

3.9 Raw time difference spectrum for an E-plane Bar. The red lines are the leading and trailing edges of the raw time difference spectrum. The blue line is centre position of the raw time difference spectrum. 57

3.10 Time difference of a $\mathrm{dE}$ bar plotted against the hit position of corresponding $\mathrm{E}$ bar that is located after the $\mathrm{dE}$ bar. From the parameters of the linear fit, the $\mathrm{dE}$ time difference is calibrated to produce the hit position along the bar. . . . . . . . . . . . . 58

3.11 Linear fit to particle hit position along bar from time difference against the raw hit position derived from the ADCs. The gradient from the straight line fit gives the attenuation length of the scintillator bar and the intercept is equal to the natural log of the ratio of the left and right ADC conversion. . . . . . . . . . . .

3.12 Top: arithmetic mean of the left and right ADCs of a dE scintillator bar versus the arithmetic mean of the left and right ADCs of a E scintillator bar. Bottom: Same as top, but the arithmetic mean has been replaced with the geometric mean of the left and right ADCs with correction for the attenuation length of the scintillator bars. . . . . . . . . . . . . . . . . 
3.13 Top-Left: dE bar TOF vs dE and E bar TOF difference for same bar number pairings. Top-Right: dE bar TOF vs E bar TOF difference for odd-even bar number pairings. The bottom histograms are the same, except the TOF offsets and dE scale factors have been implemented to make the TOF differences between $\mathrm{dE}$ and E bars and the bars on the same plane all centred around zero. . .

3.14 Diagram of a particle hit in a $\mathrm{E}$ bar and the terms necessary to calculate the in-plane angle $(\theta)$ in the hall frame. . . . . . . .

3.15 Side view of a particle trajectory through the BigBite magnetic field and auxiliary and trigger planes. The momentum and $\phi$ of the track was calculated by tracking the particle path through the scintillator planes back through the magnetic field and to the target. 64

3.16 Top: The deviation between the scattered electron energy measured in the Left HRS and the electron energy predicted using for an elastic reaction using the initial electron energy and scattering angle. Middle: The measured elastic proton momentum versus the measured proton momentum in BigBite. Bottom: The measured elastic proton out-of-plane $\phi$ versus the measured $\phi$ in BigBite. . .

3.17 Top-Left: Momentum distribution in BigBite. Top-Right: Inplane angle $\theta$. Bottom-Left: Out-of-plane angle $\phi$. Bottom-Right: Auxiliary bar number plotted against the sum of $\mathrm{dE}$ and $\mathrm{E}$ bar number. The real tracks in the main linear distribution are encircled by the red graphical cut and the other bar combinations outside this distribution are due to uncorrelated hits. . . . . . . .

3.18 The dE bar energy deposited versus E bar energy deposited for protons (smaller spectrum with a breakthrough point of $\mathrm{dE}$ and $\mathrm{E}$ bar energy deposited of $3.5 \mathrm{MeV}$ and $60 \mathrm{MeV}$ respectively) and deuterons (larger spectrum with a breakthrough point of $\mathrm{dE}$ and $\mathrm{E}$ bar energy deposited of $4.5 \mathrm{MeV}$ and $83 \mathrm{MeV}$ respectively). The wiggle in the $\mathrm{dE}$ vs E spectrums for both particles is due to the propagation step size of the particle's path, which is a little too long considering the small thickness of the $3 \mathrm{~mm}$ dE bar. . . . . . 
3.19 BigBite Geant4 simulation of dE plus E bar energy deposited versus generated momentum (top-left), approximated momentum using actual particle hit coordinates in the scintillator bars (topright), approximated momentum using the simulated detector scintillator hit coordinates (bottom-left). Bottom-right: Experimental $\mathrm{dE}$ plus E bar energy deposited versus momentum. . . . . . . . .

3.20 Top left: The deviation in simulated detected momentum from the generated momentum against the generated momentum for protons. Top right: The same as top left, but for deuterons. Bottom left: The deviation in simulated detected TOF from the generated TOF against the generated momentum for protons. Bottom right: The same as bottom left, but for deuterons. . . . . . . . . . .

3.21 Top: The deviation in simulated detected TOF from the generated TOF for protons with generated momentum cuts applied on the three histograms left to right of $150-450 \mathrm{MeV} / \mathrm{c}, 250-550 \mathrm{MeV} / \mathrm{c}$ and $350-650 \mathrm{MeV} / \mathrm{c}$ to reproduce the three kinematic settings of CK1, CK2 and CK3 respectively.Bottom: Same as the (top), but deuterons were used instead of protons in the simulation. . . . . .

$3.22-\frac{d E}{d x}$ calculated as a function of momentum for different particles for various materials in the Particle Data Group (PDG) booklet. .

3.23 Top: Calculated dE bar energy deposited against the calculated E bar energy deposited for protons and deuterons. Middle: Calculated E bar energy deposited plotted as a function of initial momentum for protons and deuterons. Bottom: Calculated dE bar energy deposited plotted as a function of initial momentum for protons and deuterons. . . . . . . . . . . . . . 
3.24 Top-left: Calculated dE energy for deuterons plotted against E bar energy calculated for protons and deuterons. This shows the calculated dE vs E energy deposited for deuterons, as in figure 3.23 and also the calculated $\mathrm{dE}$ values for deuterons plotted against the calculated E values for protons of the same momentum. The area enclosed in the red graphical cut shows that the deuterons of greater momentum than the breakthrough point $(565 \mathrm{MeV})$ are cleanly separated from the protons. Top-right: Measured E bar energy plotted against the calculated dE bar energy for deuterons. A graphical cut similar to the previous red graphical cut, was used on this histogram to identify the deuterons of high momentum. The cut cannot extend any further to include the highest distribution of deuteron momentum $(>820 \mathrm{MeV} / \mathrm{c})$ due to the high concentration of protons in this area. These missing deuterons have a greater momentum than the upper limit $(650 \mathrm{MeV} / \mathrm{c})$ of ${ }^{12} C\left(e, e^{\prime} p\right)$ missing momentum in the CK3 kinematics. Bottomleft: The calculated dE energy for deuterons and protons plotted against E bar energy calculated for deuterons. The area enclosed in the blue graphical cut shows that the deuterons of lesser momentum than the breakthrough point are cleanly separated from the protons. Bottom-Right: The measured dE bar energy plotted against the calculated E bar energy for deuterons. A graphical cut similar to the previous blue graphical cut, was used on this histogram to identify the deuterons of lower momentum than the breakthrough point. . . . . . . . . . . . .

$3.25 \mathrm{dE}$ bar energy deposited versus E bar energy deposited with deuteron cuts applied for the CK2 kinematics of $p_{\text {miss }}=400 \mathrm{MeV}$ for the ${ }^{12} C\left(e, e^{\prime} p\right)$ reaction. . . . . . . . . . . . . . .

3.26 Measured TOF minus calculated TOF of the ${ }^{12} C\left(e, e^{\prime} p p\right)$ reaction for ${ }^{12} C\left(e, e^{\prime} p\right)$ missing momenta of 300 (CK1), 400 (CK2) and 500 (CK3) $M e V / c$ (top to bottom) with no specific BigBite proton cuts. 77

3.27 Measured TOF minus calculated TOF of the ${ }^{12} C\left(e, e^{\prime} p d\right)$ reaction for ${ }^{12} C\left(e, e^{\prime} p\right)$ missing momenta of 300 (CK1), 400 (CK2) and 500 (CK3) $M e V / c$ (top to bottom) with no specific BigBite deuteron cuts. 
3.28 Measured TOF minus calculated TOF of the ${ }^{12} C\left(e, e^{\prime} p d\right)$ reaction for ${ }^{12} C\left(e, e^{\prime} p\right)$ missing momenta of 300 (CK1), 400 (CK2) and 500 (CK3) $\mathrm{MeV} / \mathrm{c}$ (top to bottom) with BigBite deuteron cuts. . . . .

3.29 Measured TOF minus calculated TOF for ${ }^{12} C\left(e, e^{\prime} p\right)$ missing momenta of 300 (CK1), 400 (CK2) and 500 (CK3) MeV/c (top to bottom). For CK1 and CK2 kinematics, the TOF difference spectrums are fitted with Gaussian superimposed on a flat background (from 0 to $60 \mathrm{~ns}$ ). For CK3, there is an additional proton peak to the left of the deuteron peak in the centre. A Gaussian fit of this proton peak is included in the fit to the background and the fit of the background + deuteron peak. . . . . . . . . . .

4.1 Graphical acceptance cuts obtained from real data used in simulation. Top: Horizontal angle versus fractional momentum for Right HRS. Middle: Vertical angle versus fractional momentum of Right HRS. Bottom: Vertical angle vs momentum measured in BigBite.

4.2 Comparison of the left HRS experimental data (red), the generated scattered electron data (blue) and simulated left HRS detected electron data (black). Top left: Momentum $(\mathrm{GeV} / \mathrm{c})$. Top right: $\theta_{t g t}(\mathrm{rad})$, vertical angle. Bottom left: $y_{t g t}$ position of reaction vertex. Bottom right: $\phi_{t g t}(\mathrm{rad})$, horizontal angle. All plots are scaled to fit on the histograms.

4.3 Comparison of the right HRS experimental data (red), the generated proton data (blue) and simulated right HRS detected proton data (black). Top left: Momentum $(G e V / c)$. Top right: $\theta_{t g t}(\mathrm{rad})$, vertical angle. Bottom left: $y_{t g t}$ position of reaction vertex. Bottom right: $\phi_{t g t}(\mathrm{rad})$, horizontal angle. All plots are scaled to fit on the histograms. . . . . . . . . . . . . . . . .

4.4 Comparison of the BigBite experimental data (red), the generated deuteron data (blue) and simulated BigBite detected deuteron data (black). Top: Momentum $(\mathrm{GeV} / \mathrm{c})$. Middle: $\theta_{t g t}(\mathrm{rad})$, vertical angle. Bottom: $\phi_{t g t}(\mathrm{rad})$, horizontal angle. All plots are scaled to fit on the histograms. BigBite does not measure the reaction vertex position and assumes it is at centre of target. . . . . . . . 87 
5.1 Differential cross section results with statistical (black error bars) and asymmetric systematic errors (red error bars) for the three kinematic settings corresponding to the missing momentum of the ${ }^{12} C\left(e, e^{\prime} p\right)$ reaction of $300(\mathrm{CK} 1), 400(\mathrm{CK} 2)$ and $500(\mathrm{CK} 3) \mathrm{MeV} / \mathrm{c} .94$ 


\section{List of Tables}

2.1 Beamline device properties. The Moeller polarimeter was not used in this experiment. . . . . . . . . . . . . . . . . 28

2.2 HRS characteristics. . . . . . . . . . . . . . . . . . . . 32

3.1 The initial cuts applied to HRS variables. . . . . . . . . . . 50

3.2 Yield results using the parse random subtraction method and the yield using a flat background and Gaussian fits of the ${ }^{12} C\left(e, e^{\prime} p d\right)$ reaction for each kinematic setting with statistical error first and then systematic error on the final yield results. . . . . . . . . . .

4.1 Resolution and acceptance characteristics of the HRS and BigBite spectrometers. . . . . . . . . . . . . . .

4.2 Acceptance calculations for the three kinematic settings. $N_{\text {sim }}$ are the number of simulated events that pass the acceptance of all three detectors out of 100 million generated events $\left(N_{g e n}\right)$ for the three kinematic settings of CK1, CK2 and CK3. Acc is the the acceptance calculated using 4.1. $N_{s i m-U A}$ and $N_{s i m-L A}$ are the number of simulated events from the simulations of the upper $\left(A_{c-U A}\right)$ and lower $\left(A_{c-L A}\right)$ possible total acceptance. . . . . . . . . .

4.3 The total charge measured by the beam charge monitors and the luminosites with statistical and systematic errors for each kinematic setting after the application of normalisation correction factors.

5.1 Differential cross section results with statistical and asymmetric systematic errors respectively for the three kinematic settings corresponding to the missing momentum of the ${ }^{12} C\left(e, e^{\prime} p\right)$ reaction of 300 (CK1), $400(\mathrm{CK} 2)$ and $500(\mathrm{CK} 3) \mathrm{MeV} / \mathrm{c}$. . . . . . 


\section{Chapter 1}

\section{Introduction}

Nuclear physics is the study of the structure and the dynamics of the atomic nucleus and its constituent nucleons (protons and neutrons). The strong force, described by Quantum Chromodynamics, which binds together the quarks and gluons of the nucleons, creates a residual nuclear force that holds the protons and neutrons of the atomic nucleus together by the exchange of mesons. This residual nucleon-nucleon $(\mathrm{NN})$ force mediated by the exchange of composite mesons rather than quarks and gluons was first described by Yukawa [11] in 1935. Although the properties of the NN force are well known at a phenomenological level, only recently has it been possible to construct a nuclear wavefunction ab-initio using such forces. Thus explanations of nuclear properties have traditionally used simplified ansatz such as the Shell Model, where nucleons move fairly independently of one another in a mean field generated by the other nucleons. This model has been very successful in describing many nuclear properties, however proton knock-out experiments, such as $\left(e, e^{\prime} p\right)$ [12], have found the depleted occupancy of shells below the Fermi level in closed shell nuclei and that there is an underestimation of high momentum and high energy components of the spectral function.

These problems of the shell model are understood to be caused by its failure to describe Short Range Correlations (SRC) and Long Range Correlations (LRC) of nucleons due to its assumption of the independent nucleons moving in a mean field. Short Range Correlations are essentially the high momentum interactions between nucleons, taking place at short distances of less than $1 \mathrm{fm}$ (the order of the nucleon radius). Here the wavefunctions of the individual nucleons overlap strongly and the NN interaction is repulsive. While medium-range effects are described in terms of pion exchange, shorter ranges use heavier mesons such as the $\rho$ meson. The present work is based on a JLab experiment which was primarily 
designed to measure the cross section ratio of ${ }^{12} C\left(e, e^{\prime} p p\right)$ events to ${ }^{12} C\left(e, e^{\prime} p n\right)$ events and the cross section ${ }^{12} C\left(e, e^{\prime} p\right)$ and extract the relative probability of $p-n$ and $p-p$ SRCs. However, this experiment also recorded data on the ${ }^{12} C\left(e, e^{\prime} p d\right)$ reaction, which has some sensitivity to 3 Nucleon Forces (3NF) and is the main focus of this thesis.

The ability to perform very accurate calculations in few-nucleon systems has emerged in the last few years and has revolutionised the understanding of light nuclei. Modern ab-initio calculations can now handle realistic NN forces models, developed mainly from partial wave analysis of nucleon-nucleon scattering experiments. Modern NN potentials used in these calculations include the Argonne $V_{18}$ (AV18) and CD Bonn Potential and CD Bonn $+\Delta$ model. These potentials first developed in the 1990s, were born out of the work on meson models from the 1960s and 70s [13]. Although these modern potentials are largely phenomenological in nature, the parameters are fitted to reproduce accurate NN scattering data with very high precision and are continuously updated. However, for $A \geq 3$ nuclei, calculations using these potentials showed an underestimation of binding energy, for ${ }^{3} \mathrm{He}$ by $\sim 0.5-0.9 \mathrm{MeV}$ [14] and for ${ }^{4} \mathrm{He}$ by $\sim 2.0-4.2$ $\mathrm{MeV}$ [14-16]. To fix this discrepancy, 3 Nucleon Forces (3NF) were added using fits to the $A=3$ binding energy and more recently also to describe energies of bound and low-lying resonant states of p-shell nuclei [17-22].

Fujita and Miyazawa first predicted $2 \pi$ exchange between three nucleons with $\Delta$-isobar excitation half a century ago [23] and today it is thought to be the dominant 3NF meson exchange mechanism. A number of other 3NF mechanisms have been proposed, including short range components involving $N^{*}$ excitation and exchange of heavier mesons such as the $\rho$ and $\omega$. The development of $3 \mathrm{NF}$ models is also being influenced by recent insights from chiral perturbation theory $[18,24-26]$, which include mechanisms involving one pion exchange with two nucleons undergoing a contact interaction and three nucleons in a contact interaction, as well as the standard $2 \pi$ exchange diagram.

Chiral perturbation theory (CHPT) is an effective field theory which embodies the chiral structure of QCD at low energy, where the running QCD coupling constant is large and a perturbation expansion in powers of the coupling constant breaks down. When the chiral symmetry of QCD is spontaneously broken, the corresponding Goldstone bosons can be identified as pions. Pions, the lightest hadron $\left(m_{\pi} \sim 140 \mathrm{MeV}\right)$ are referred to as pseudo-Goldstone bosons due to their approximation as massless Goldstone bosons. Their interaction is weak at low momenta, allowing the expansion of the scattering amplitude in powers of 
small external momenta and the pion mass. This means CHPT can describe the interaction of pions and between pions and matter fields (nucleons, $\rho$ mesons, $\Delta$ resonances, ...) in a systematic way. In the pion and pion-nucleon sector, this peturbative scheme works well because the interaction vanishes at vanishing external momenta in the chiral limit, where quark mass tends to zero.

However, the strong interaction between nucleons makes it difficult to apply the standard methods of CHPT in the purely nucleonic sector. This is due to the interaction between nucleons remaining strong in the chiral limit at vanishing three-momenta of external nucleons, creating a non-perturbative expansion in CHPT. One method to overcome this problem was proposed by Weinberg, was to apply CHPT to the kernel of the corresponding integral equation for the scattering amplitude, creating an effective NN potential [27,28]. From this idea, Weinberg formally demonstrated for the first time the established view of the order of importance of few nucleon forces, namely: 2 Nucleon interactions, 3 Nucleon interactions, 4 Nucleon interactions and so on. It has since been shown that $3 \mathrm{NF}$ terms in the Next-Leading-Order (NLO) chiral expansion cancel and the first non-vanishing contribution to $3 \mathrm{NF}$ appears in at the Next-to-Next-LeadingOrder expansion [29-32].

\subsection{Previous Measurements}

One of the important areas of study of the properties of the $3 \mathrm{NF}$ in recent years has been the nucleon-deuteron system at low to intermediate energies. Several institutes have carried out experiments to measure the cross section and various polarisation observables of the different possible reactions in this system. These include $\overrightarrow{p d}, \overrightarrow{n d}$ and $\overrightarrow{d p}$ elastic scatting, $\overrightarrow{p d}$ and $\overrightarrow{d p}$ captures and $\overrightarrow{p d}$ and $\overrightarrow{d p}$ break-up reactions. Initially, $3 \mathrm{NF}$ effects were looked for in low energy $p d$ and $n d$ scattering processes, but without success. Precise measurements, yielded accurate data which could be reproduced by NN force calculations, meaning 3NF contributions were not required. Since then, experiments have moved on to using intermediate energies after Witala et al [33], pointed out this might be a more fertile area for $3 \mathrm{NF}$ effects in the cross section minimum region of the centre of mass (C.M.) angular distribution at backward angles (from around $90^{\circ}-150^{\circ}$ or greater) of Nucleon - deuteron elastic scattering above nucleon laboratory energies of $E_{N}^{L a b} \sim 60 \mathrm{MeV}$.

In a recent review of this field of research [34], it noted that the excellent fits 
of Faddeev type calculations employing modern NN + 3NF apply to experimental cross sections and analysing powers below $135 \mathrm{MeV}$, as these calculations are only strictly applicable at energies below the pion production threshold. Unfortunately, a consistent description of equivalent data at higher energies was not possible and the review concludes that the $3 \mathrm{NF}$ models need to go beyond using the $2 \pi$ exchange only and possibly incorporate ideas from CHPT and also improve the relativistic treatment.

\subsubsection{Measurement of 2- and 3-Nucleon Short Range Cor- relation Probabilities in Nuclei}

The inclusive electron scattering $A\left(e, e^{\prime}\right)$ cross section ratios of ${ }^{3} \mathrm{He},{ }^{12} \mathrm{C}$ and ${ }^{56} \mathrm{Fe}$ to ${ }^{3} \mathrm{He}$ were measured at $1<x_{B}<3$, with four-momentum transfer $Q^{2} \geq 1.5$ $\mathrm{GeV}^{2}$ in an effort to measure to the probabilities of 2 and 3 nucleon SRCs [35]. Bjorken $x_{B}=\frac{Q^{2}}{2 M_{p} v}$, the fractional momentum of the struck nucleon and has a maximum value equal to the number of correlated nucleons, was required to be greater than 1.3 to suppress scattering off nucleons of low to typical momenta. Short range correlations were isolated by using high transfered energies and momenta. If center of mass $(\mathrm{cm})$ motion of the SRC in the nuclear mean field is ignored, the nuclear cross section due to electrons scattering from nucleons in $2 \mathrm{~N}$, $3 \mathrm{~N}, 4 \mathrm{~N}$, etc... SRC configurations can written as [36,37]:

$$
\sigma_{A}\left(Q^{2}, x_{B}\right)=\sum_{j=2}^{A} A \frac{a_{j}(A)}{j} \sigma_{j}\left(Q^{2}, x_{B}\right)
$$

where $\sigma_{A}$ and $\sigma_{j}$ are the cross sections of electron-nucleus and electron-jnucleon-correlation interactions respectively and $a_{j}(A)$ is the ratio of the probability for a given nucleon to belong to correlation $j$ in nucleus A and to belong to a nucleus containing a correlation of $j$ nucleons. The ratios of cross sections of atomic mass numbers $A$ and $A^{\prime}$ are expected to have the form of $\frac{\sigma(A)}{\sigma\left(A^{\prime}\right)}=\frac{A^{\prime}}{A} \cdot \frac{a_{j}(A)}{a_{j}\left(A^{\prime}\right)}$, be independent of $x_{B}$ and $Q^{2}$ and have discrete values for different $\mathrm{j}$, due to $j$ nucleon SRC dominating at $j<x_{B}<j+1$ and the probabilities of $j$-nucleon SRC dropping rapidly with $j$. 


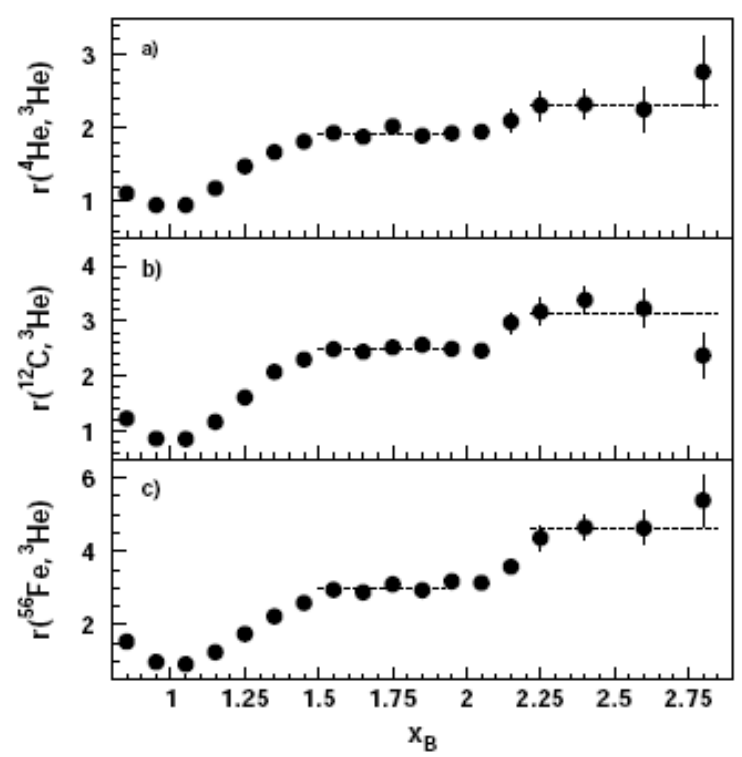

Figure 1.1: Weighted cross section ratios of (a) ${ }^{4} \mathrm{He}$, (b) ${ }^{12} \mathrm{C}$ and (c) ${ }^{56} \mathrm{Fe}$ to ${ }^{3} \mathrm{He}$ as a function of $x_{B}$ for $Q^{2}>1.4 \mathrm{GeV}^{2}$. The horizontal dashed lines indicate the $N N$ and $3 N$ scaling regions used to calculate the per-nucleon probabilities for 2and $3 N$ SRCs in nucleus $A$ relative to ${ }^{3} \mathrm{He}$.

The results (see fig. 1.1) show this 'scaling' of the ratio in the plateau regions of $1.5<x_{B}<2$ for $2 \mathrm{~N}$ SRC and $x_{B}>2.25$ for $3 \mathrm{~N}$ SRC. These features are consistent with theoretical expectations that NN SRCs dominate the nuclear wave function at missing momentum $p_{m} \gtrsim 300 \mathrm{MeV}$ and $3 \mathrm{~N}$ SRC dominate at $p_{m} \gtrsim 500 \mathrm{MeV}$.

\subsubsection{Study of Three-Nucleon Mechanisms in the Photo- disintegration of ${ }^{3} \mathrm{He}$}

The cross section of the ${ }^{3} \mathrm{He}(\gamma, p p) n$ reaction was measured over wide proton energy and angular range $\left(200 \mathrm{MeV} \leq E_{\gamma} \leq 800 \mathrm{MeV}\right.$ and $\left.20^{\circ} \leq \theta_{p}^{l a b} \leq 160^{\circ}\right)$ at MAMI [6]. The differential cross sections for ${ }^{3} \mathrm{He}(\gamma, p d)$ reaction was also measured as part of the same experiment (see 1.1.3). The yield for the $\gamma\left({ }^{3} \mathrm{He}\right) \rightarrow p p n$ reaction is shown in fig. $1.2(\mathrm{i})$ has a large peak at $E_{\gamma} \simeq 320 \mathrm{MeV}$, indicating that in this region, the reaction is produced by the intermediate $\Delta$ excitation with no other structure observed in the second resonance region up to $800 \mathrm{MeV}$. The full curve of the Laget model (1998) [38] integrated over the experimental acceptance has reasonable agreement with the yield measurement. Events coming from the $2 N$ absorption mechanisms were estimated and then subtracted using a model 
of Laget (see dotted line in fig. 1.2(i)) and the total $3 N$ photoabsorption cross section was obtained on the assumption of a uniform $3 N$ phase-space distribution (see fig. 1.2(ii)). The obtained 3N absorption strength in ${ }^{3} \mathrm{He}$ represents $36 \%$ of the $2 N$ strength, which agrees with the order of magnitude measured in pion absorption experiments [39-41]. The full curve of the Laget model integrated over the experimental acceptance has reasonable agreement with the yield measurement. Looking at the contributions of the different reaction processes in the model $(2 N, 3 N(2-s t e p)$ and $3 N(2 \pi)$ mechanisms $)$, it is clear that $3 \mathrm{~N}$ cross section in this model is dominated by the ' $3 \mathrm{~N}(2$-step)' process.
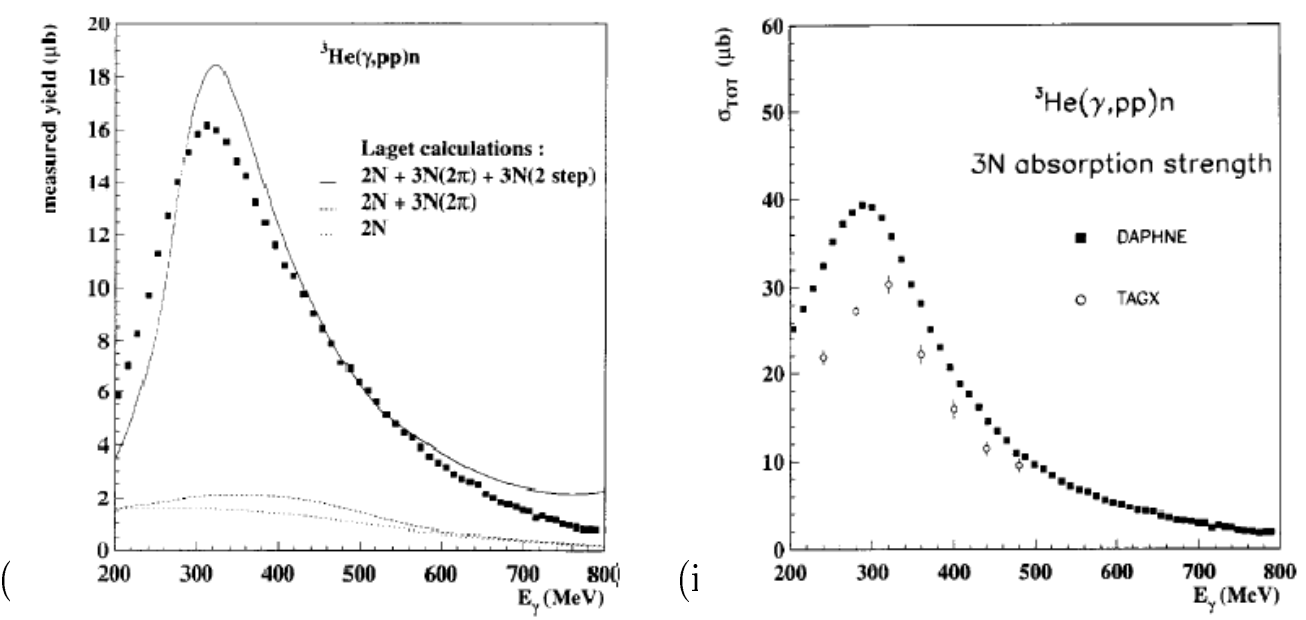

Figure 1.2: (i) The total cross section of the $\gamma^{3} H e \rightarrow p p n$ channel integrated over the detector acceptance (black squares) is compared to the theoretical predictions of Laget. (ii) The total cross section for $3 N$ photoabsorption estimated from the experimental data compared to results obtained by the TAGX experiment, which measured the same reaction channel for a photon energy range from $200-500$ $\mathrm{MeV}$ using the spectrometer TAGX, which has a solid angle for protons of $\pi \mathrm{sr}$. Discrepancies in the comparison of results are probably due to the Laget model used in this experiment and its ability to calculate the $2 N$ contribution in good agreement with previous data.

There are two different possible $3 N(2-$ step $)$ mechanisms that were predicted to account for most of the $3 \mathrm{~N}$ photoabsorption cross section according to the Laget model. The first mechanisms is the the exchange of two pions that are always off mass shell and the other mechanism is where an on-mass-shell pion is photoproduced by the absorption the photon on a nucleon and is reabsorbed by the remaining pair. Before the signature of either of these different mechanisms were obtained, contributions to the cross section by Final State Interactions (FSI) were illuminated by creating a triangular Dalitz plot for the ${ }^{3} \mathrm{He}(\gamma, \mathrm{ppn})$ data for 
$E_{\gamma}=400 \pm 25 \mathrm{MeV}$ (see fig. 1.3(i)). FSI effects show up most prominently in the two densely populated areas and were removed by selecting the central region delimited by two parallel lines. The invariant mass $m_{x}$ for the exchange pion was reconstructed for the $3 N(2-s t e p)$ process and was plotted as a function of $m_{x}^{2} / m_{\pi}^{2}$ in fig. 1.3(ii), for $E_{\gamma}=300 \mathrm{MeV}$.
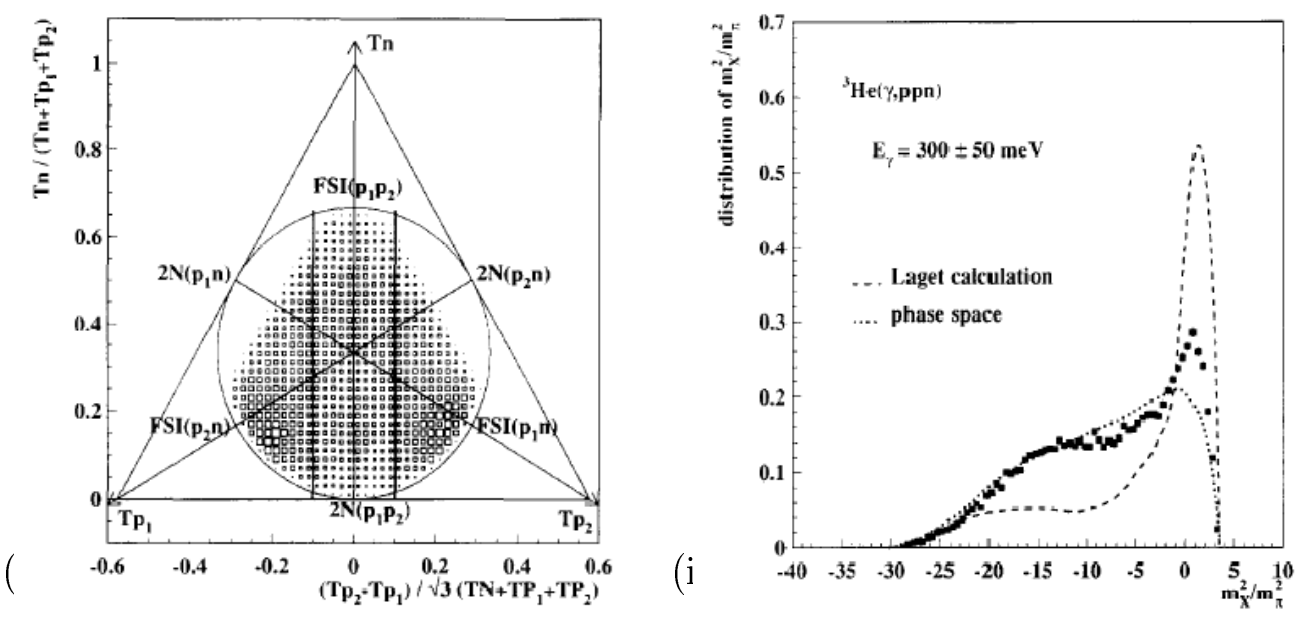

Figure 1.3: (i) Triangular Dalitz plot at $400 \mathrm{MeV}$ : the three axes are the c.m. kinetic energies of the proton $_{1}$, proton 2 , and neutron normalised to the total c.m. energy. Events in the region between the 2 parallel lines were selected for the study of the $3 \mathrm{~N}$ mechanisms. (ii) Distribution, at $300 \mathrm{MeV}$, of the invariant mass of the object $\mathrm{X}$ which is exchanged between the proton and the neutron-pair.

The peak about $m_{x}^{2} / m_{\pi}^{2} \sim 1$ in fig. $1.3($ ii) is a clear indicator that the $3 N$ photodisintegration of ${ }^{3} \mathrm{He}$ in the $\Delta$ region has a substantial component from the photoproduction on one nucleon and the reabsorption of the produced pion by the two other nucleons. Above $500 \mathrm{MeV}$, this mechanism was not observed.

\subsubsection{Two-body photodisintegration of ${ }^{3} \mathrm{He}$ between 200 and $800 \mathrm{MeV}$}

The differential cross sections for ${ }^{3} \mathrm{He}(\gamma, p d)$ reaction at photon energies between 200 and $800 \mathrm{MeV}$ at all proton c.m. angles between $35^{\circ}$ and $145^{\circ}$ were measured at MAMI using the large acceptance detector DAPHNE [5]. Fig. 1.4 shows the cross section as a function of photon energy for proton c.m. angles of $60^{\circ}$ and $90^{\circ}$. The rapid decrease in the measured cross section agrees with previous data and contrasts with deuteron photodisintegration, where a $\Delta$ excitation is clearly seen. This process is thought to be suppressed in the ${ }^{3} H e(\gamma, p d)$ reaction due to 


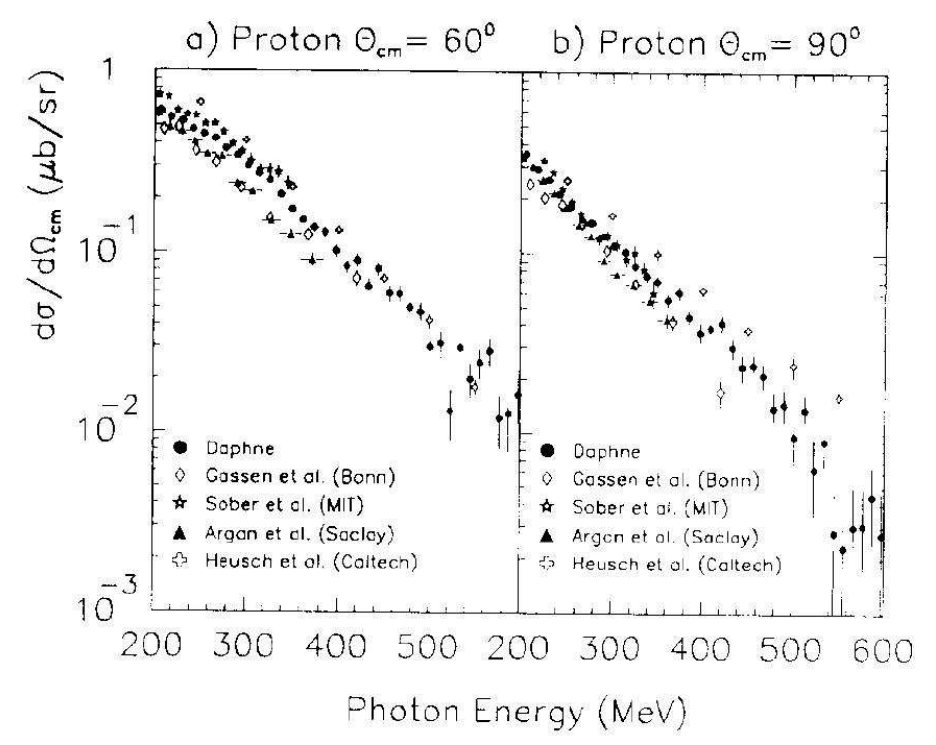

Figure 1.4: The excitation curves at proton c.m. angles of $60^{\circ}$ (a) and $90^{\circ}$ (b) obtained in this experiment compared with previous measurements.

the influence of the form factor linked to the formation of a deuteron in the final state as previously demonstrated by $[42,43]$ and [1].

Fig. 1.5 shows the cross section as a function of proton c.m. angle for a series of fixed photon energies. Up to a proton c.m. angle of $90^{\circ}$, there is a steep decrease in the differential cross section due to the dominance of the two body photodisintegration mechanisms. However, at more backward angles, a bump in the cross section is observed precisely in the region where three body mechanisms are thought to play an important role [1]. At these backward angles the Laget calculations using one and two body mechanisms and also the contribution of 3 body mechanisms displayed in fig. 1.5, show that the one and two body mechanisms do not reproduce the magnitude or form of the measured cross section. The calculations that include $3 \mathrm{~N}$ mechanisms reproduce the qualitive form of the cross section but overestimates the magnitude of the data. This overestimation may be due a number of reasons in the calculation, including the absence of two possible reaction mechanisms but the most likely explanation is that the Fermi motion of the nucleon at the photonuclear vertex is not taken into account.

\subsubsection{Three-nucleon mechanisms in photoreactions}

The ${ }^{12} C(\gamma, p p n)$ reaction was measured for $E_{\gamma}=150-800 \mathrm{MeV}$ at MAMI [44] and was compared to calculations using the Valencia model (VM) [45-47], which 

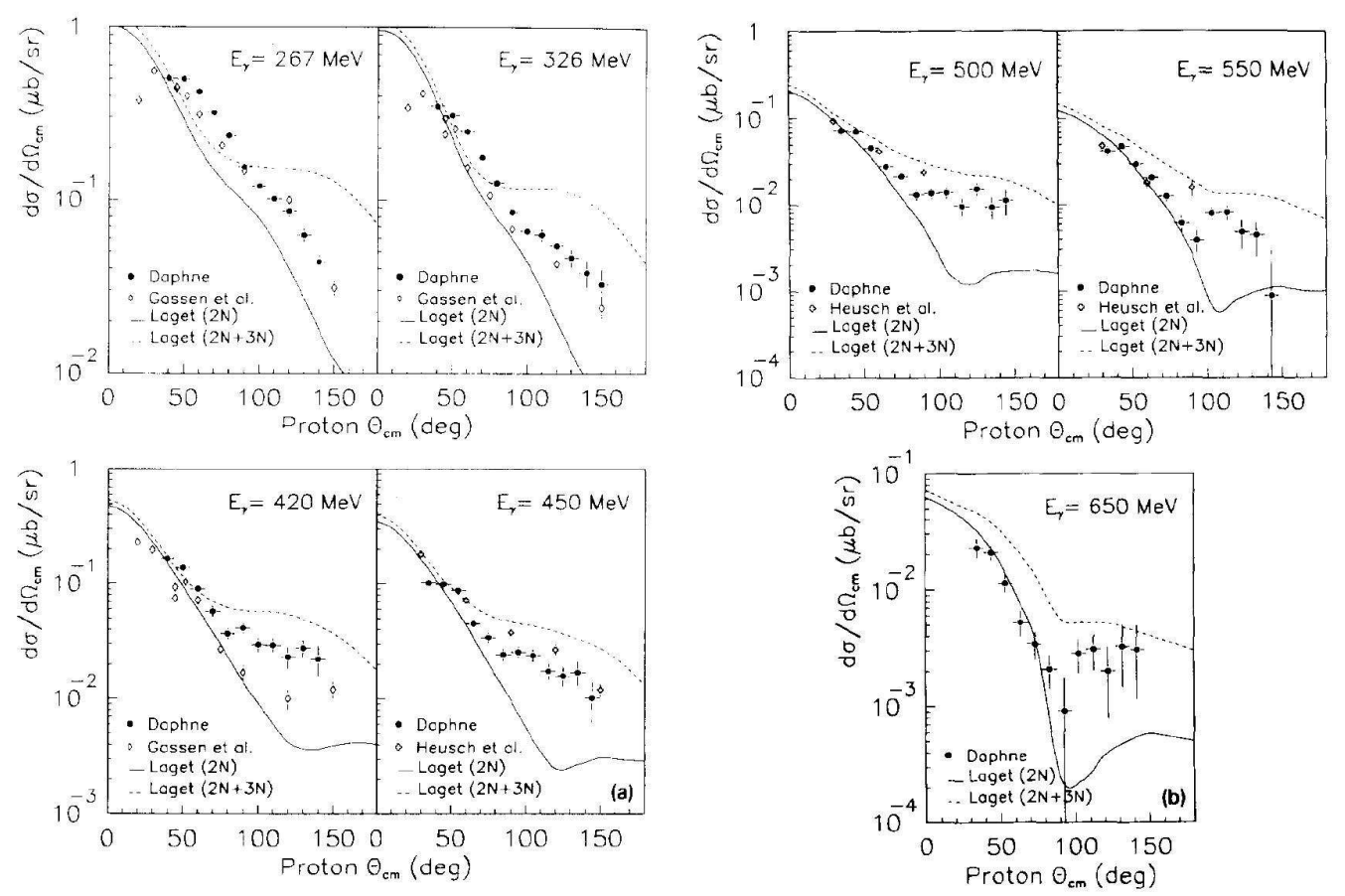

Figure 1.5: Angular distributions at photon energies of 267, 326, 420, 450, 500, 550 and $650 \mathrm{MeV}$, obtained in this experiment compared to previously published data (up to $550 \mathrm{MeV}$ ) and to theoretical calculations of Laget [1]. The continuous curve gives the contribution of one and two body mechanisms while the dashed curve also includes the contribution of three body processes. 
is a microscopic many-body model describing photon-nucleus interactions. The VM model allows the separation of the contributions to the total photonuclear cross section from different photon absorption mechanisms through Cutosky rules. A local density approximation is used to give a realistic matter distribution for nuclei. From this theoretical approach, the cross sections for the absorption of the photon by two nucleons $(2 N)$, three nucleons $(3 N)$ and pion production processes $(N \pi$ and $N N \pi)$ are obtained. Both pion and shorter range meson exchanges are included in the $3 N$ mechanism, but resonances higher than the $\Delta$ are not. The Valencia model also accounts for final state interactions (FSI) for both the nucleons and pions produced in the absorption process.

In fig. 1.6, the measured ${ }^{12} C(\gamma, p p n)$ cross section is shown as a function of the three-body missing energy. The cross section is strong near to the separation energy $(32 \mathrm{MeV})$ of the reaction corresponding to the $A-3$ system being left close to its ground state. At higher missing energies the cross section is also significant, meaning there is a large contribution from processes which leave the $A-3$ system highly excited, which may indicate that more than three nucleons are emitted. Similar features are observed in $\left(\pi^{+}, p p p\right)$ reactions [48].

The results of the VM simulation of the ${ }^{12} C(\gamma, p p n)$ reaction is also shown in fig. 1.6. Initial $N \pi$ process contributions in the VM simulation were separated into cases where the pion is reabsorbed $(N \pi+\mathrm{ABS})$ or when the pion was emitted $(N \pi+$ EMIT $)$. This was due to the strong evidence that the $N \pi+$ ABS process in ${ }^{12} C$ is overestimated by the VM model $[49,50]$. The predictions for this process were reduced by a factor of 0.3 in order for the model to agree with the ${ }^{12} C(\gamma, p)$ data [50] for the backward proton angles from this same experiment. Comparisons $[49,50]$ with ${ }^{12} C(\gamma, n p)$ data at backward proton angles become much closer in agreement when the same factor is used. The predicted contributions from all the different reaction mechanisms (after $N \pi+$ ABS strength reduction) are separated by different shades and stacked upon each other (see the key in fig. 1.6 for identification). The sum of all the reaction mechanisms without the reduction of the $N \pi+$ ABS process shows that the VM prediction gives a poor description of the experimental data. However, after the reduction of the $N \pi+$ ABS process, the model gives a good description of the size and shape of the measured data over a wide range of missing energy and $E_{\gamma}$. The VM model predicts that the $3 \mathrm{~N}$ process is greatest at low missing energy $(<100 \mathrm{MeV})$, even though it still contributes less than $1 / 3$ of the cross section in this region. 


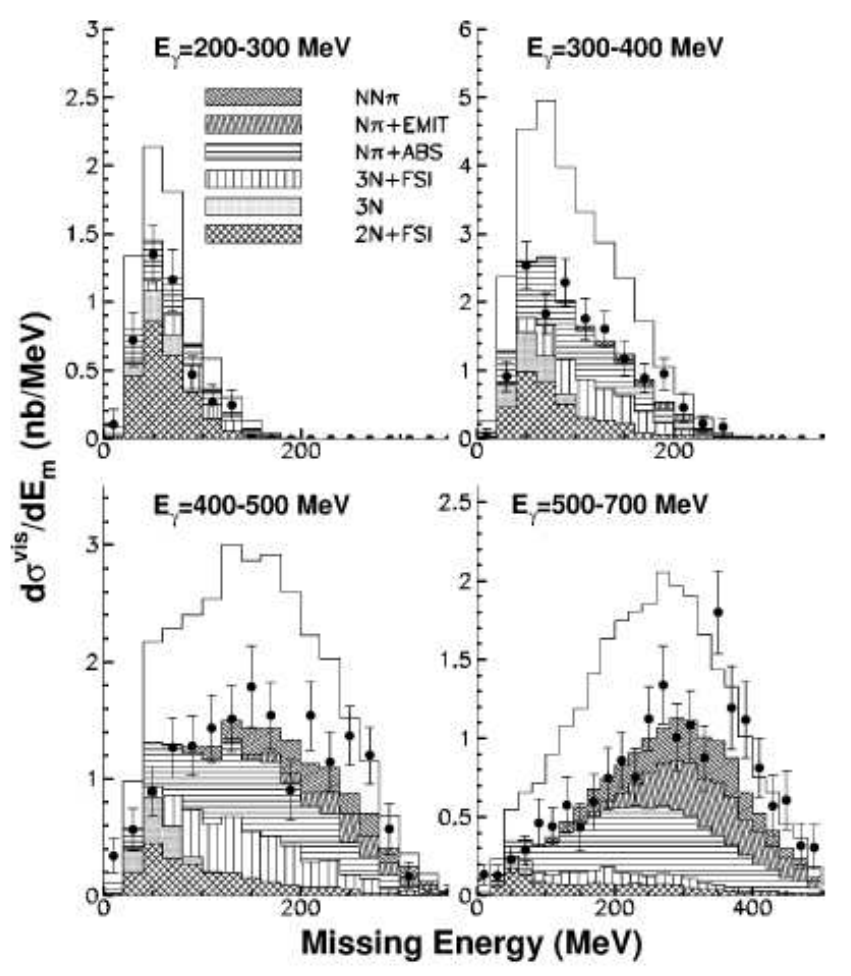

Figure 1.6: Three-body missing energy distributions for the ${ }^{12} C(\gamma, p p n)$ reaction. The shaded histograms show the VM predictions for the different reaction mechanisms indicated by the key on the figure. The solid line shows the total cross section without the reduction of the $N \pi+$ ABS prediction.

To further isolate the $3 N$ contribution, the contribution of $(N \pi+\mathrm{ABS})$ process was further reduced by reconstructing the invariant mass of the pion. If Fermi motion and nucleon FSI are neglected, the invariant mass $\left(M_{X}\right)$ in an assumed $\gamma+N \rightarrow N+X$ reaction can be reconstructed from the measured 4 -vectors of the photon and one of the detected nucleons, i.e. $M_{X_{i}}^{2}=$ $\left(E_{\gamma}+m_{N_{i}}-E_{N_{i}}\right)^{2}-\left(p_{\gamma}-p_{N_{i}}\right)^{2}$ for $i=1,2,3$. Therefore, for on-shell pion photoproduction $\left(M_{X i} / m_{\pi}\right)^{2}$ should be $\sim 1$ for the nucleon involved.

The comparison of the predicted $\left(M_{X} / m_{\pi}\right)^{2}$ distributions with the experiment is shown in fig. 1.7. The missing energy was restricted to $<100 \mathrm{MeV}$ for both the experiment and model, to reduce contributions from processes involving FSI but still including all the strength from the direct $3 N$ process. The $\left(M_{X} / m_{\pi}\right)^{2}$ distributions of the neutron and more forward proton are shown but the other more backward proton is omitted as it is much less likely to have arisen from an initial $N \pi$ process due to kinematic reasons. The experimental data for the proton and neutron have an invariant mass peak around $m_{\pi}$ and a negative tail of strength extending into the negative $\left(M_{X} / m_{\pi}\right)^{2}$ values. The distributions of 
the reaction mechanisms in the VM simulation that are also displayed in fig. 1.7 (in the $2 \mathrm{D}$ scatter plot and in the projections) show the tendency of the $N \pi$ + ABS contribution to cluster near to $\left(M_{X} / m_{\pi}\right)^{2}=1$. The VM model is in good agreement with the experimental data in the peak and negative tail, but only after the reduction in strength of the $N \pi+$ ABS process. The predicted contribution of $3 N$ and $2 N+F S I$ processes in the peak is reasonable but there is more relative strength in the negative tail.

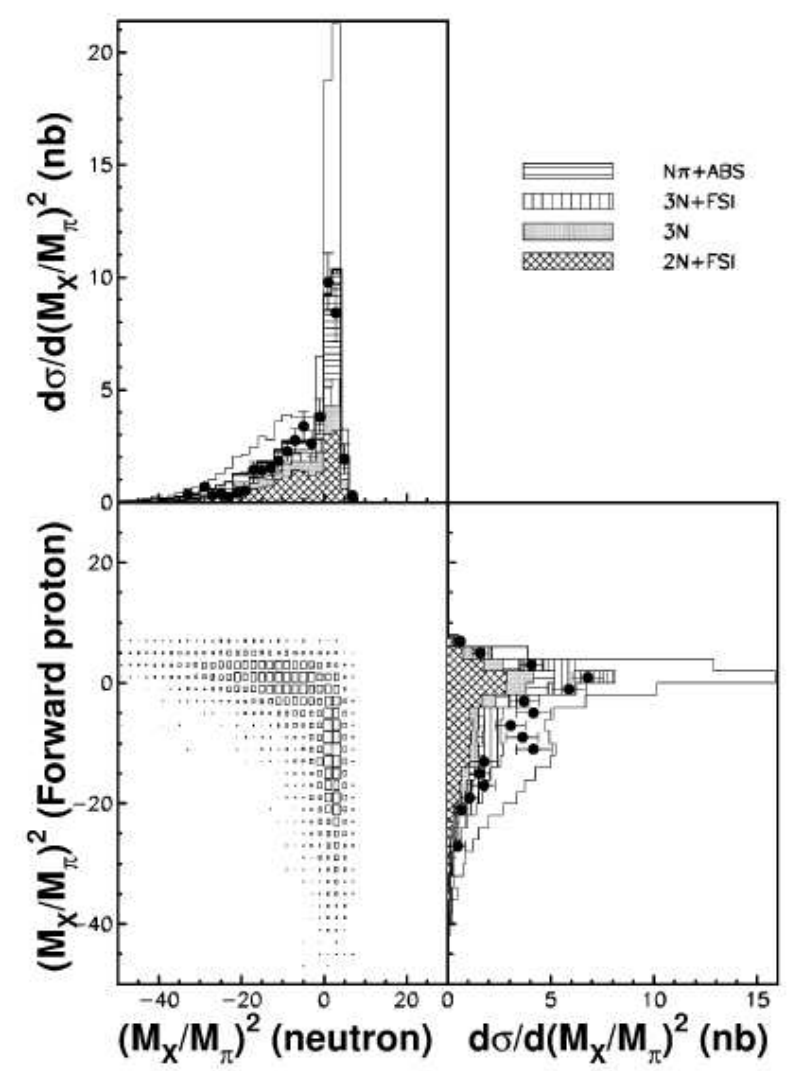

Figure 1.7: Measured and predicted $\left(M_{X} / m_{\pi}\right)^{2}$ distributions for the ${ }^{12} C(\gamma, p p n)$ reaction at missing energy of $\leq 100 \mathrm{MeV}$ and $E_{\gamma}=150-500 \mathrm{MeV}$. Bottom left panel shows the Valencia model predictions of $\left(M_{X} / m_{\pi}\right)^{2}$ for the neutron versus the forward proton. The projections onto the $x$ and $y$ axes (shaded histograms) are compared with the experimental data in the top left and bottom right panels. The solid line shows the total cross section without the reduction of the $N \pi+$ ABS prediction.

The photon energy dependence of the cross section was also used to analyse the contributions of the different reaction mechanisms (see top half of fig. 1.8), using cuts of missing energy of $\leq 50 \mathrm{MeV}, \leq 100 \mathrm{MeV}$ and $\geq 100 \mathrm{MeV}$. The less than $50 \mathrm{MeV}$ missing energy cut restricts the excitation energy of the residual 
nucleus and selects the knockout of three (1p) shell nucleons, however all shell combinations of $3 \mathrm{~N}$ knockout are included by cutting below $100 \mathrm{MeV}$ missing energy which also includes nearly all the $3 N$ strength predicted by the VM. In this region, the VM (after $N \pi+$ ABS strength reduction) is in good agreement with the experimental data and accounts for the observed strong resonance structure in the $\Delta$ region. For missing energy greater than $100 \mathrm{MeV}$, the VM also gives a good description of the experimental data up to photon energies below $\sim 450 \mathrm{MeV}$, where the extra strength in the measured data compared to the VM prediction may be due to $2 \pi$ production processes which are not included in the model.

To reduce the sensitivity to the initial strength of the $N \pi+$ ABS process in the photon energy dependence of the cross section (see lower half of fig. 1.8), a restriction of $\left(M_{X} / m_{\pi}\right)^{2} \leq-1.5$ was applied to the neutron and the more forward proton (see fig. 1.7). This cut effectively removes a major part of the predicted $N \pi+$ ABS strength for missing energy below $100 \mathrm{MeV}$ and boosts the relative strength of $3 N$ and $2 N+F S I$ processes. The VM predictions are in good agreement in shape and magnitude with the data and for $E_{\gamma}$ below $\sim 250 \mathrm{MeV}$, where only $2 N+F S I$ process is expected to contribute. The good agreement is a sign of the good description of this process by the model. For photon energies around $400 \mathrm{MeV}$, almost half of the cross section is predicted to come from the $3 N$ process and the other process at this low missing energy, $2 N$ knockout, is well understood. The high strength of the direct $3 N$ absorption in these kinematical conditions and the different $E_{\gamma}$ dependence for $3 N$ and $2 N+F S I$ processes, produces a good opportunity for extracting information on the strength and $E_{\gamma}$ dependence of the $3 N$ component. 


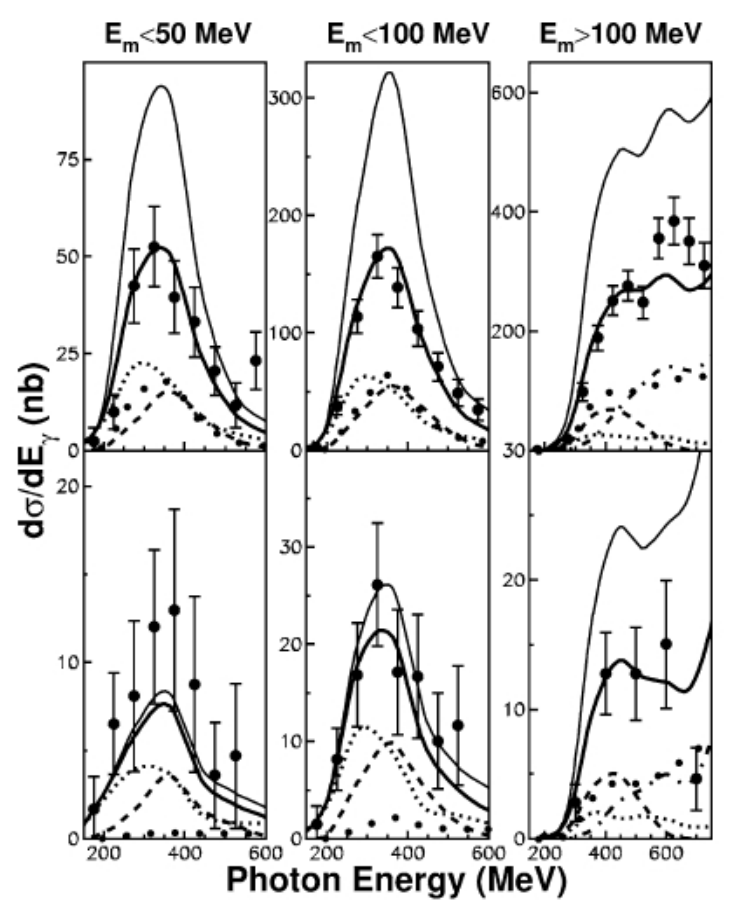

Figure 1.8: The ${ }^{12} C(\gamma, p p n)$ cross section as a function of photon energy presented for three missing energy regions indicated in the figure. The lower figures have the additional cut $\left(M_{X} / m_{\pi}\right)^{2} \leq-1.5$ for the neutron and the most forward-angle proton. The total prediction of the (modified) Valencia model is shown by the thick solid line and the separate contributions from the $2 N+$ FSI, $3 N$ (with or without FSI), $N \pi+$ ABS, and $N N \pi / N \pi+$ EMIT processes are shown by the short dash, long dash, dotted and dot-dashed lines respectively. The thin solid line shows the total VM without reducing the $N \pi+$ ABS strength.

\subsubsection{Dependence of the ${ }^{12} C(\vec{\gamma}, p d)$ Reaction on Photon Linear Polarisation}

The ${ }^{12} C(\vec{\gamma}, p d)$ reaction was measured for the first time at MAMI for $E_{\gamma}=170$ to $350 \mathrm{MeV}$, to investigate the three-body interaction in a heavier nucleus than most previous $(\vec{\gamma}, p d)$ reaction measurements, which have mainly been performed using light nuclei such as ${ }^{3} \mathrm{He}$ [51]. As with the ${ }^{3} \mathrm{He}$, three nucleon forces in ${ }^{12} \mathrm{C}$ are related to the direct 3 -nucleon knockout mechanisms in the $(\gamma, p d)$ reaction. However, there are other contributions from a variety of other reaction mechanisms such as initial photon absorption by a single nucleon, 2 nucleons and two step $3 \mathrm{~N}$ processes such as real pion production on one nucleon and re-absorption by a nucleon pair. By measuring the $(\vec{\gamma}, p d)$ photon asymmetry in conjunction with the cross section (see fig. 1.9) in a nucleus of more typical (larger) nuclear den- 
sity and with wider ranges of relative angular momentum and spin couplings, this experiment highlighted the role of the different reaction mechanisms in photon induced proton-deuteron knockout when heavy nuclei compared to light nuclei.
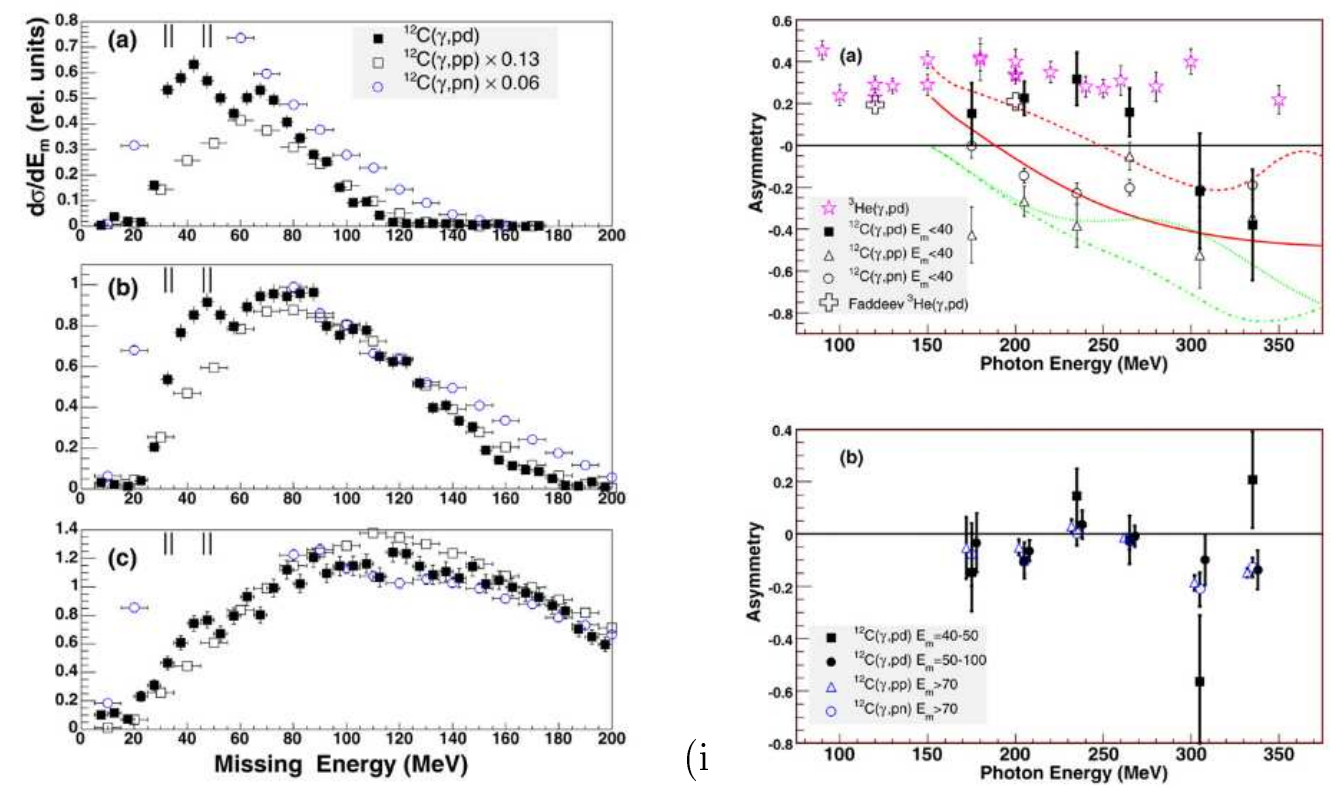

Figure 1.9: (i) Cross section for the $(\gamma, p d),(\gamma, p p)$ and $(\gamma, p n)$ reactions versus missing energy. The data presented for $E_{\gamma}$ bins of (a) 170-220 MeV, (b) 220-280 $\mathrm{MeV}$ and (c) 280-350 MeV. (ii) (a) The ${ }^{12} C(\vec{\gamma}, p d),{ }^{12} C(\vec{\gamma}, p n)$ and ${ }^{12} C(\vec{\gamma}, p p)$ photon asymmetry integrated over $\theta_{p}=51^{\circ}-120^{\circ}$ for the missing energy cuts indicated in (i) (a), (b), (c). The errors are statistical only. The experimental results [2] for ${ }^{3} \mathrm{He}(\vec{\gamma}, p d)$ at $\theta_{p}=90^{\circ}$ and $110^{\circ}$ and the Faddeev predictions [3] at $\theta_{p}=110^{\circ}$ are also shown. The red (solid) and green (dot-dash) lines give the asymmetry at $\theta_{\pi}=55^{\circ}$ from the MAID [4] calculations for $p\left(\vec{\gamma}, \pi^{0}\right) p$ and $p\left(\vec{\gamma}, \pi^{+}\right) n$ respectively. The corresponding predictions without including $\Delta$ (1232) are shown by the red (dashed) and green (dotted) lines. (b) Comparison of the ${ }^{12} C(\vec{\gamma}, p d),{ }^{12} C(\vec{\gamma}, p n)$ and ${ }^{12} C(\vec{\gamma}, p p)$ asymmetry at higher missing energy.

For missing energies greater than $40 \mathrm{MeV}$, the asymmetry of ${ }^{12} \mathrm{C}(\vec{\gamma}, p d)$ is small or negative and is comparable to ${ }^{12} C(\vec{\gamma}, N N)$ reactions, suggestive of similar reaction mechanisms. Models of $(\gamma, N N)$ in this missing energy region suggest the cross section is made up largely from detection of two nucleons from a two-step $3 \mathrm{~N}$ process or the initial absorption of the photon by a 2 nucleon pair followed by the final state interactions. The similarities in asymmetries and the cross sections in terms of missing energy, greater than $40 \mathrm{MeV}$, of the ${ }^{12} C(\vec{\gamma}, p d)$ and ${ }^{12} C(\vec{\gamma}, N N)$ reactions can be explained if the primary reaction mechanisms are the same, but one of the outgoing nucleons picks up an additional nucleon from the residual nucleus. Recent research has shown that a cut in 
recoil momentum $p_{R}<300 \mathrm{MeV} / \mathrm{c}$ for missing energy between 40 and $70 \mathrm{MeV}$ enhances the asymmetry of the ${ }^{12} C(\vec{\gamma}, p p)$ reaction as the cut samples direct knockout processes [52].

The missing energy region less than $40 \mathrm{MeV}$ emphasises $(\gamma, p d)$ reactions leading to the ground state and low lying energy states of ${ }^{9} \mathrm{Be}$. The asymmetry is positive for photon energies up to $280 \mathrm{MeV}$, and above this energy, in the $\Delta$ resonance region, it becomes negative. The ${ }^{3} H e(\vec{\gamma}, p d)$ asymmetry is similar in magnitude and sign up to $270 \mathrm{MeV}$, but it does not become negative. The low missing energy ${ }^{12} C(\vec{\gamma}, p d)$ reactions are the most likely to contain contributions from reaction mechanisms that involve only the detected nucleons and leave the residual nucleus as a spectator. The similarity of the asymmetry for this region of missing energy in both the ${ }^{12} \mathrm{C}(\vec{\gamma}, p d)$ and ${ }^{3} \mathrm{He}(\vec{\gamma}, p d)$ reactions may indicate a similar reaction mechanism in both nuclei. The $\vec{\gamma} N \rightarrow N \pi$ asymmetry was examined using the MAID code [4] to calculate both $p\left(\vec{\gamma}, \pi^{+}\right) n$ and $p\left(\vec{\gamma}, \pi^{0}\right) p$ processes, which are possible first steps in a two-step $3 \mathrm{~N}$ process. The $\Delta$ contribution to the inital pion production vertex for the two-step process is supressed due to an isospin restriction [1], therefore the MAID calculations with and without the $\Delta$ contribution are displayed in 1.9 (ii). Although the $p\left(\vec{\gamma}, \pi^{0}\right) p$ MAID prediction with no $\Delta$ contribution is comes close to the $(\vec{\gamma}, p d)$ asymmetry, the MAID cross sections are largest for the $p\left(\vec{\gamma}, \pi^{+}\right) n$ process for which the asymmetry is negative. This means that it is unlikely that the asymmetries for the ${ }^{12} \mathrm{C}(\vec{\gamma}, p d)$ at low missing energies or for ${ }^{3} \mathrm{He}(\vec{\gamma}, p d)$ can be reproduced by this form of two-step $3 \mathrm{~N}$ mechanism. This adds weight to the idea that the low lying states in ${ }^{9} B e$ resulting from the ${ }^{12} C(\vec{\gamma}, p d)$ reaction are unlikely to have occurred through a two-step $3 \mathrm{~N}$ mechanism at low missing energy and is a possible route to access $3 \mathrm{NFs}$ in the (1p) shell.

\subsubsection{The $(\gamma, p d)$ reaction in ${ }^{12} C$}

The ${ }^{12} C(\gamma, p d)$ reaction was measured with tagged photons in the energy range of $150-400 \mathrm{MeV}$ at MAMI [53] with a overall energy resolution of $\sim 6 \mathrm{MeV}$. The missing energy of the $(\gamma, p d)$ reaction is defined as $E_{m}=E_{\gamma}-T_{p}-T_{d}-T_{R}=$ $S_{p d}+E_{x}$, where $E_{\gamma}, T_{p}$ and $T_{d}$, are respectively, the energies of the tagged photon and detected proton and deuteron. $T_{R}$ is the kinetic energy of the recoiling nucleus ${ }^{9} \mathrm{Be}$, which is derived from $E_{\gamma}, T_{p}$ and $T_{d}$ and the angles of the detected particles using conservation of energy and momentum. $S_{p d}=31.7 \mathrm{MeV}$ is the reaction separation energy and $E_{x}$ is the excitation energy of the recoiling nucleus. The 
missing energy spectra for different bands of tagged photon energy is shown in fig. 1.10. From examination of these spectra, indications of the initial shells of the emitted nucleon were drawn. Namely, the peak at low photon energies for this ${ }^{12} C(\gamma, p d)$ reaction is not seen in the ${ }^{12} C(\gamma, p p)$ reaction [54-56], but is more similar to the ${ }^{12} C(\gamma, p n)$ reaction. This has been attributed to possible contributions from the photon interaction and knockout of p-shell nucleons, while the rest of the nucleus acts like a spectator. Between $E_{m} \simeq 50-80 \mathrm{MeV}$, the small bump noticeable for forward angles between photon energies of $200-250$ $\mathrm{MeV}$ may be due to involvement of s-shell nucleons. As photon energy and proton angle increases there is a shift from low to high $E_{m}$. Therefore, it is likely the strength of different reaction mechanisms contributions changes with photon energy and proton angle.

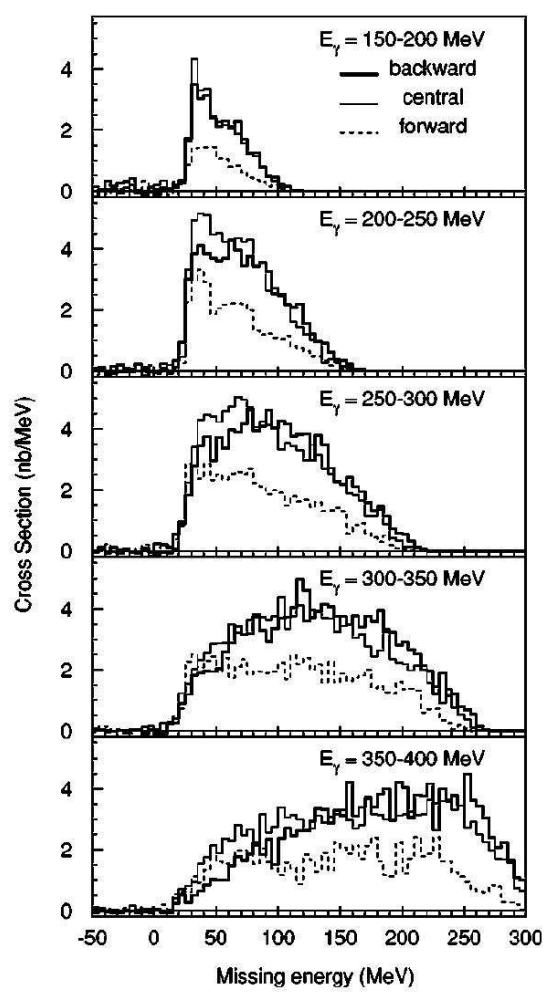

Figure 1.10: Missing energy spectra for the ${ }^{12} C(\gamma, p d)$ reaction for backward (thick), central (thin), and forward (dashed) proton angles.

In fig. 1.11, the photon energy dependence of the cross section was compared with that of the ${ }^{3} \mathrm{He}(\gamma, p d)$ reaction from the DAPHNE detector [5], for different proton angles. The proton angles were obtained using the deuteron angles, measured by four TOF detectors, and calculated using two body kinematics of 
the ${ }^{12} \mathrm{C}(\gamma, p d)$ reaction, in which the initial quasi- ${ }^{3} \mathrm{He}$ and residual ${ }^{9} \mathrm{Be}$ nucleus were at rest. To make allowance for some Fermi motion, the corresponding proton bin was taken as $\theta_{p} \pm 10^{\circ}$. To reduce the effect of final state interactions, a missing energy cut of $E_{m}<44 \mathrm{MeV}$ was applied, where all three nucleons should come directly from the $p$ shell. The ${ }^{12} \mathrm{C}$ and ${ }^{3} \mathrm{He}$ are plotted at different scales to allow comparison. Above photon energies of $\sim 250 \mathrm{MeV}$ the shapes of the ${ }^{12} \mathrm{C}$ and ${ }^{3} \mathrm{He}$ cross sections are similar. The differences at lower photon energies and forward angles are explained to be due to the deuteron threshold in TOF and to the effect of Fermi motion on the reconstruction of the proton angles, both of which are more likely to occur at low photon energy and forward proton angles. The ${ }^{3} \mathrm{He}(\gamma, p p) n$ cross section [6] displayed for $\theta_{p}=88^{\circ}$ exhibits a strong enhancement in the region of the $\Delta(1232)$ resonance which is not seen in either of the ${ }^{3} \mathrm{He}(\gamma, p d)$ or ${ }^{12} \mathrm{C}(\gamma, p d)$ reaction. This is thought to be due to the same isospin selection rule effects as in the ${ }^{3} \mathrm{He}(\gamma, p d)$ reaction [1].

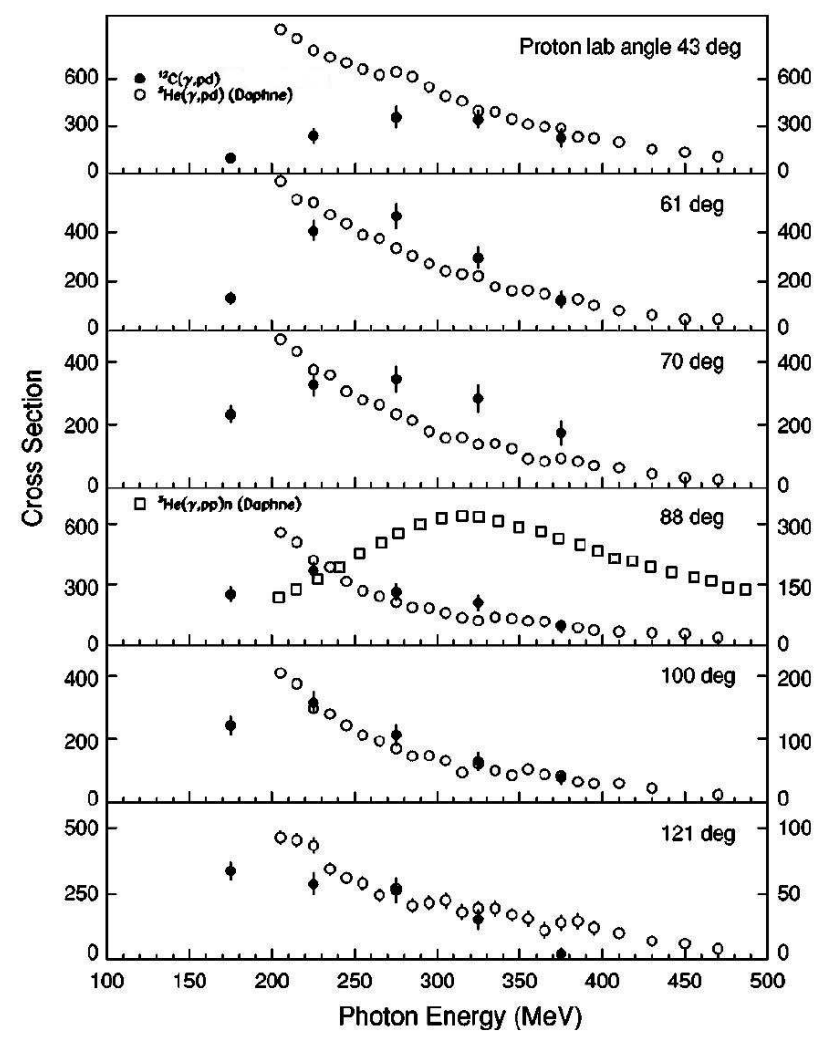

Figure 1.11: Photon energy dependence of the ${ }^{12} C(\gamma, p d)$ reaction for $E_{m}<44$ $\mathrm{MeV}$ (left scale) compared to that for the ${ }^{3} \mathrm{He}(\gamma, p d)$ reaction [5] (right scale) and the ${ }^{3} \mathrm{He}(\gamma, p p) n$ reaction [6] (total cross section, right scale times 50). The ${ }^{12} \mathrm{C}$ data are expressed in units of $n b / s r^{2}$, the ${ }^{3} \mathrm{He}(\gamma, p d)$ data are in $n b / s r$, and the ${ }^{3} \mathrm{He}(\gamma, p p) n$ data are in $n b$. 
In models that assume the interaction of only three nucleons, while the rest of the nucleus spectates and no FSI occurs, the recoil momentum distribution $P_{r}=p_{\gamma}-p_{p}-p_{d}$ is equal in magnitude and opposite in direction to the initial total momentum of the three emitted nucleons. At a basic level, the three (momentum space) nucleon wave functions can also be folded together to produce the $P_{r}$ distributions [57]. Elton-Swift [58] wave functions were used to model $P_{r}$ distributions for $E_{m}<44 \mathrm{MeV}$ where three $p$-shell wave functions are appropriate (see fig. 1.12 (i)) and for higher $E_{m}$, where one or more $s$-shell wave functions are required (see fig. 1.12 (ii)). The first calculations shown in the figures assume the three nucleons are at zero separation and therefore in a relative $S$ state.

(i)
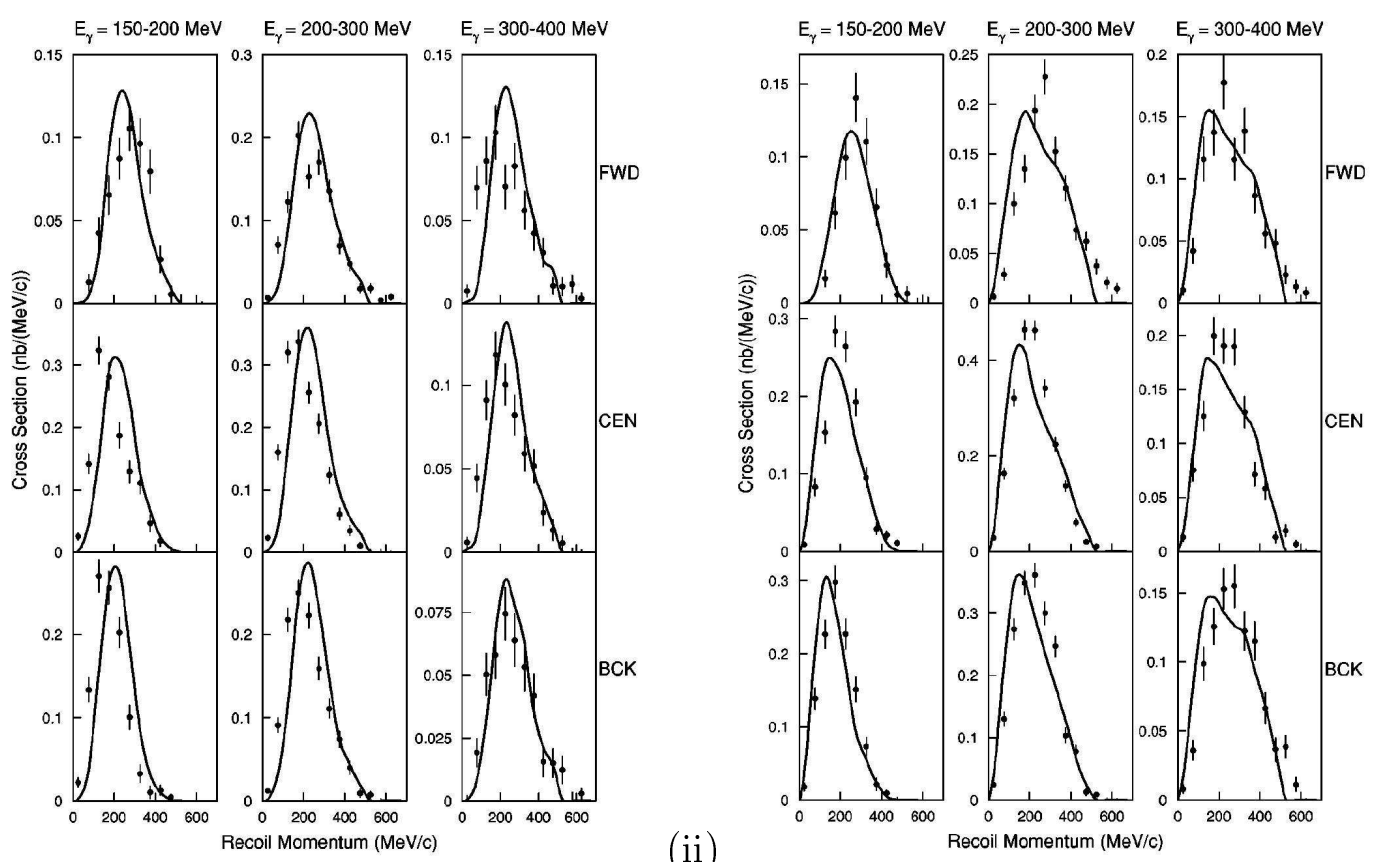

Figure 1.12: Recoil momentum distributions for the ${ }^{12} C(\gamma, p d)$ reaction for (i) $E_{m}<44 \mathrm{MeV}$ and (ii) $E_{m} 44-70 \mathrm{MeV}$. The curves show the distributions predicted by the simple model described in the text. FWD, CEN, and BCK refer to the proton detector angles of $22.7^{\circ}-101.1^{\circ}, 51.3^{\circ}-128.6^{\circ}$, and $79.0^{\circ}-156.7^{\circ}$ respectively.

The model produces a reasonably good description of the shape and strength of the $P_{r}$ distributions despite the lack of FSI effects included. The zero separation approximation, used to calculate the initial nucleon momentum distributions, is thought to be responsible for the lack of strength predicted for low recoil momentum at all photon energies for $E_{m}<44 \mathrm{MeV}$ and also for the too much strength at higher $E_{m}$. Similar effects have been noticed in ${ }^{12} C(\gamma, p p)$ reactions [54,55], but with the models predicted strength reversed for the two $E_{m}$ regions due to 
the parity reversal brought about by the extra $p$-shell nucleon in the ${ }^{12} C(\gamma, p d)$ reaction. The problem of the zero separation approximation for the ${ }^{12} C(\gamma, p p)$ experiments has been put down to the exclusion of $P$ (and higher) wave admixtures to the relative wave function of the active nucleons in the initial state. The inclusion of these wave admixtures is expected to improve the model's agreement with this experiment. For example, at low missing energy $E_{m}<44 \mathrm{MeV}$, the assumption of three active nucleons in a relative $S$ state gives terms with $L=1,3$ for $(1 p)^{3}$ knockout. However, if there is a contribution from a nucleon in a relative $P$ state, this would require additional $L=0,2$ terms and because most of the strength at low recoil momentum comes from the $L=0$ term, the agreement with the experiment should be improved.

\subsubsection{Investigation of the Exclusive ${ }^{3} H e\left(e, e^{\prime} p n\right)^{1} H$ Reac- tion}

The cross sections for the ${ }^{3} H e\left(e, e^{\prime} p n\right)^{1} H$ reaction were measured at energy transfers of 220 and $270 \mathrm{MeV}$ for several momentum transfers ranging from 300 to 450 $\mathrm{MeV} / \mathrm{c}$ at MAMI [59]. Exclusive two nucleon knockout reactions using electron scattering of the type $A\left(e, e^{\prime} p N\right) A-2$ is a very direct method of studying the correlations between nucleons induced by $N N$ interactions. Due to the differences in scalar and tensor interactions in isospin states $T=0$ and $T=1, p p$ knockout reactions probe predominantly the repulsive short-range components while $p n$ knockout reactions probe the attractive strong tensor components of the meson-exchange contribution at intermediate and long distances. The two nucleons can be ejected when the virtual photon couples to one nucleon of the correlated pair of nucleons via the one-body hadronic currents. Other processes that can also knockout two nucleons that could contribute to the cross section include the interaction of the virtual photon with two-body hadronic currents, such as meson exchange currents (MEC) or isobar currents (IC) and also interactions involving all three nucleons $(3 \mathrm{NF})$ or final state interactions (FSI). One way of learning more about these contributing processes is to compare measurements of both $\left(e, e^{\prime} p p\right)$ and $\left(e, e^{\prime} p n\right)$ reactions. The ${ }^{3} H e\left(e, e^{\prime} p p\right) n$ reaction measured at AmPs was measured at similar kinematics [60-62] allows useful comparison to the measured ${ }^{3} \mathrm{He}\left(e, e^{\prime} p n\right)^{1} \mathrm{H}$ reaction.

Using ${ }^{3} \mathrm{He}$ as the target nucleus has two main advantages. Firstly, the final state is a single nucleon in its ground state meaning that the reconstruction of the final state is simple and the detector resolution is not critical. Secondly, the 
break-up cross section can be calculated exactly using theoretical models [6365]. The measured cross sections were compared to the results of non-relativistic continuum Faddeev calculations using the Argonne V18 (AV18) and Bonn-B (BB) $N N$ potentials [64], which contain mechanisms for photon absorption on one or two of the nucleons in the target but are limited in their treatment of MEC, IC and 3 NF. The calculations are only applicable for photon energies below pion production threshold and FSI effects were fully taken into account. There were two types of calculations, the first type only used the one-body hadronic current operator, while the second also employed the two-body current operator for $\pi$ and $\rho$ mesons.

The ${ }^{3} \mathrm{He}\left(e, e^{\prime} p n\right)$ cross section as a function of missing momentum is shown in fig. 1.13 for the $\mathrm{A} 1(\omega=220 \mathrm{MeV}, q=375 \mathrm{MeV} / \mathrm{c})$ kinematic setting. There are four other kinematic settings used in this experiment corresponding to different transferred energy $\omega$ and transferred momentum $q$ and are labelled as A2 (270, 375), B2 (270, 450), Y1 (220, 300), Z2 (270, 330) using the same ( $\omega$ in $M e V, q$ in $M e V / c$ ) convention as before. The decreasing exponential shape of the theoretical cross sections with increasing missing momentum $p_{m}$ is similar to that of the measured cross section, but both potentials overpredict the experimental cross section by a factor of about 5 at $p_{m} \leq 80 \mathrm{MeV} / \mathrm{c}$. As $p_{m}$ increases the discrepencies between theoretical predictions and the experimental data decrease until they are in rough agreement within the large experimental error bars at $p_{m} \approx 200 \mathrm{MeV} / \mathrm{c}$. The inclusion of MECs increases the cross section by about $10 \%$ up to $p_{m}=200 \mathrm{MeV} / \mathrm{c}$, increasing to about $60 \%$ at 350 $\mathrm{MeV} / \mathrm{c}$. At these kinematics, the calculations indicate the ${ }^{3} \mathrm{He}\left(e, e^{\prime} p n\right)$ cross section is dominated by the one-body hadronic current term. For the ${ }^{3} \mathrm{He}\left(e, e^{\prime} p p\right)$ reaction [61], calculations using the $\mathrm{BB}$ potential are slightly below the data at low $p_{m}$ and are a factor of 5 lower at $p_{m}=200 \mathrm{MeV} / \mathrm{c}$. This is a complete contrast to overpredictions by the theoretical calculations of the ${ }^{3} \mathrm{He}\left(e, e^{\prime} p n\right)$ cross section. Both measured cross sections also do not fall as quickly as predicted with increasing $p_{m}$. 


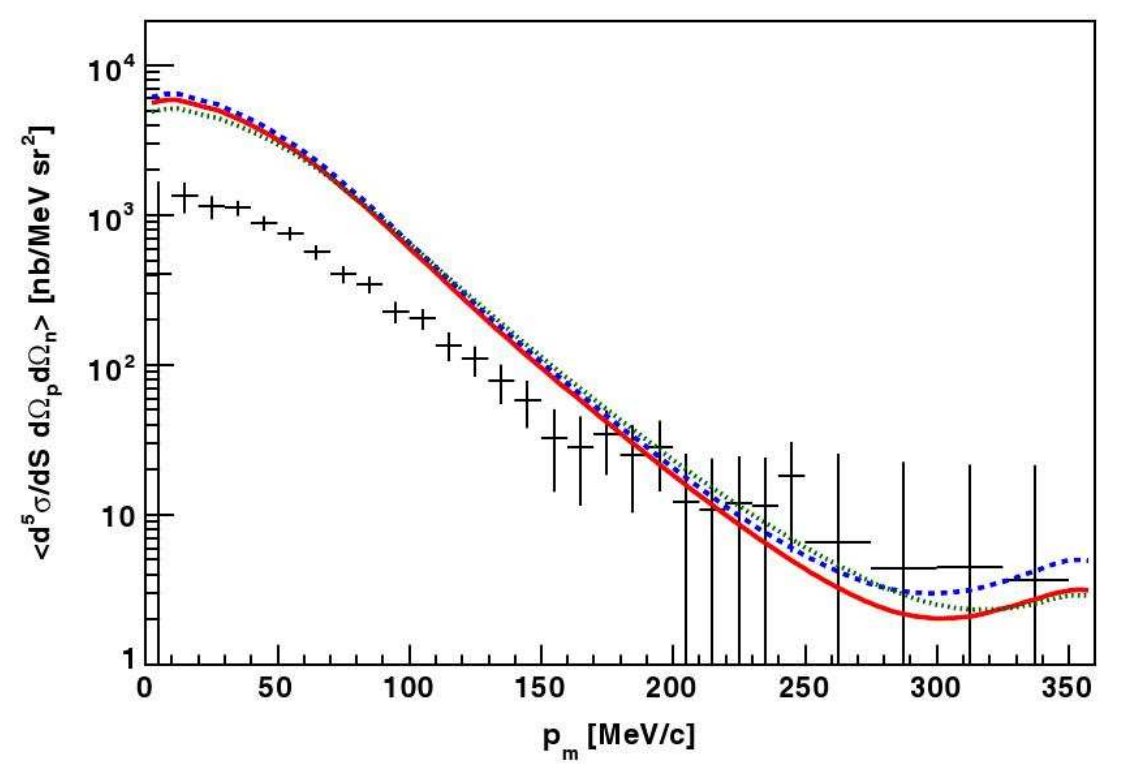

Figure 1.13: The ${ }^{3} H e\left(e, e^{\prime} p n\right)$ reaction cross section averaged out over the experimental acceptance, as a function of missing momentum for the A1 kinematic setting. The solid red (dotted green) curve shows the theoretical cross section calculated using a one-body hadronic current operator and the AV18 (BB) $N N$ potential. The dashed blue line results from the AV18 potential when MECs are also included.

In fig 1.14, the ${ }^{3} \mathrm{He}\left(e, e^{\prime} p n\right)$ cross section as a function of transfered momentum $q$ for the ranges $235 \leq \omega \leq 265 \mathrm{MeV}$ and $50 \leq p_{m} \leq 100 \mathrm{MeV} / \mathrm{c}$ where effects due to $3 \mathrm{NF}$ might be expected to be small. The experimental cross section increases smoothly with $q$ by a factor of 2 from $q=300$ to $450 \mathrm{MeV} / \mathrm{c}$. The theoretical calculations overpredict the measured cross sections by a factor of $2-3$ at $q=320 \mathrm{MeV} / \mathrm{c}$ to a factor of about 5 at $q=450 \mathrm{MeV} / \mathrm{c}$. The inclusion of MECs increases the measured cross section by about $30 \%$ at $q=320$ $\mathrm{MeV} / \mathrm{c}$ and as $q$ rises the increase in cross section falls to about $5 \%$ at $q=450$ $\mathrm{MeV} / c .{ }^{3} \mathrm{He}\left(e, e^{\prime} p p\right)$ data for a similar $p_{m}$ range is much better described by the calculations with just a $q$ independent underprediction of $20 \%$. 


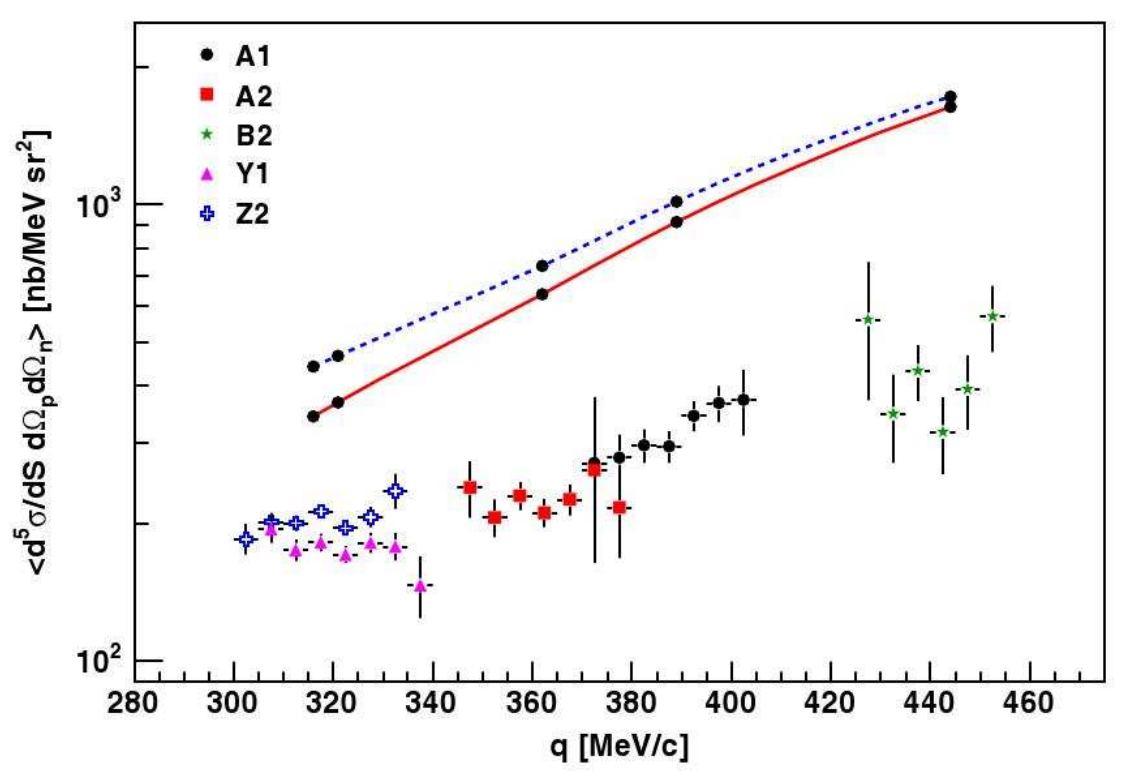

Figure 1.14: The ${ }^{3} H e\left(e, e^{\prime} p n\right)$ reaction cross section shown as a function of $q$ for $50 \leq p_{m} \leq 100 \mathrm{MeV} / \mathrm{c}$ and $235 \leq \omega \leq 265 \mathrm{MeV}$. Results from different kinematic settings are shown by the types of markers for the data points. The line convention is the same as in fig. 1.13. The theoretical cross section was calculated at the points indicated by the black circles.

The same theoretical calculations applied to the ${ }^{3} \mathrm{He}\left(e, e^{\prime} p p\right)$ and ${ }^{3} \mathrm{He}\left(e, e^{\prime} p n\right)$ reactions, provide a much better description of the ${ }^{3} \mathrm{He}\left(e, e^{\prime} p p\right)$ reaction than they do of the ${ }^{3} \mathrm{He}\left(e, e^{\prime} p n\right)$ reaction at low $p_{m}$ and also of the $q$ dependence of the cross section. This suggests that the $p n$ correlations in the probed kinematical regime are probably not well described by the theoretical calculations. Another possibility or in addition to any theoretical problems describing $p n$ correlations could be the role of $3 \mathrm{NF}$ processes which were not included in the theoretical model, or the incomplete treatment of MECs and the omission of ICs, which play a more significant role in $p n$ knockout than in $p p$ knockout. 


\section{Chapter 2}

\section{Experiment E01015}

The Jefferson Laboratory Hall A experiment E01015, "Studying the Internal Small-Distance Structure of Nuclei via the Triple Coincidence (e,e'p+N) Measurement", ran from December 2004 to March 2005 [66]. A schematic of the experimental kinematics is shown in fig 2.1. A continuous-wave electron beam with energy of $4.627 \mathrm{GeV}$ and a current up to $\sim 40 \mu \mathrm{A}$ was scattered off a 0.25 $\mathrm{mm}{ }^{12} \mathrm{C}$ foil target tilted at an angle of $20^{\circ}$ to the beam. This knocks out a proton and a recoiling nucleon or deuteron. The scattered electrons of central momentum $3.724 \mathrm{GeV} / \mathrm{c}$ were detected in a High Resolution Spectrometer (HRS) at an angle of $19.5^{\circ}$ on the left side of the beamline. These kinematics correspond to the quasi-free knockout of single protons with transferred 3-momentum $|\vec{q}|=1.65 \mathrm{GeV} / \mathrm{c}$, transferred energy $\omega=0.865 \mathrm{GeV}$, four momentum (squared) $Q^{2}=2(\mathrm{GeV} / \mathrm{c})^{2}$ and Bjorken $x_{B}=\frac{Q^{2}}{2 M \omega}=1.2$.

The knocked-out protons were detected at three different angles of $40.1^{\circ}, 35.8^{\circ}$ and $32.0^{\circ}$ on the right side of the beamline in the second optically nominally identical HRS with corresponding central momenta of $1.45,1.42$ and $1.36 \mathrm{GeV} / \mathrm{c}$ respectively. These kinematic settings corresponding to the measurement of reaction ${ }^{12} C\left(e, e^{\prime} p\right)$ at missing momenta of $0.31,0.41$, and $0.52 \mathrm{GeV} / \mathrm{c}$. A third large acceptance spectrometer called BigBite positioned at an angle of $99.0^{\circ}$ on the right side of the beamline was used to detect recoiling protons and deuterons in coincidence with the $\left(e, e^{\prime} p\right)$ events. A neutron detector was placed directly behind the BigBite spectrometer so that recoiling neutron events could also be measured. The experimental trigger was designed so that all ${ }^{12} C\left(e, e^{\prime} p\right)$ and ${ }^{12} C\left(e, e^{\prime} d\right)$ events within the spectrometer acceptance were accepted. Any coincident proton, neutron or deuteron in BigBite were recorded, but a coincidence was not required. This allowed simultaneous measurements of $\left(e, e^{\prime} p\right),\left(e, e^{\prime} d\right)$, 
$\left(e, e^{\prime} p p\right),\left(e, e^{\prime} p n\right),\left(e, e^{\prime} p x\right)$ and $\left(e, e^{\prime} d x\right)$ differential cross sections to be made in a kinematic domain where short-range correlations are expected to dominate over other competing reactions mechanisms that include meson exchange currents, delta excitation and final state interactions. The analysis of two nucleon knockout channels $[67,68]$ and ${ }^{12} C\left(e, e^{\prime} p\right)$ reaction [9] has been performed elsewhere. This thesis presents the first analysis of the ${ }^{12} C\left(e, e^{\prime} p d\right)$ reaction at high energy and momentum transfer. The cross section for this process is low and the experimental design was not optimised for this channel. Thus only a limited statistical precision was obtained and this work represents a pilot study.

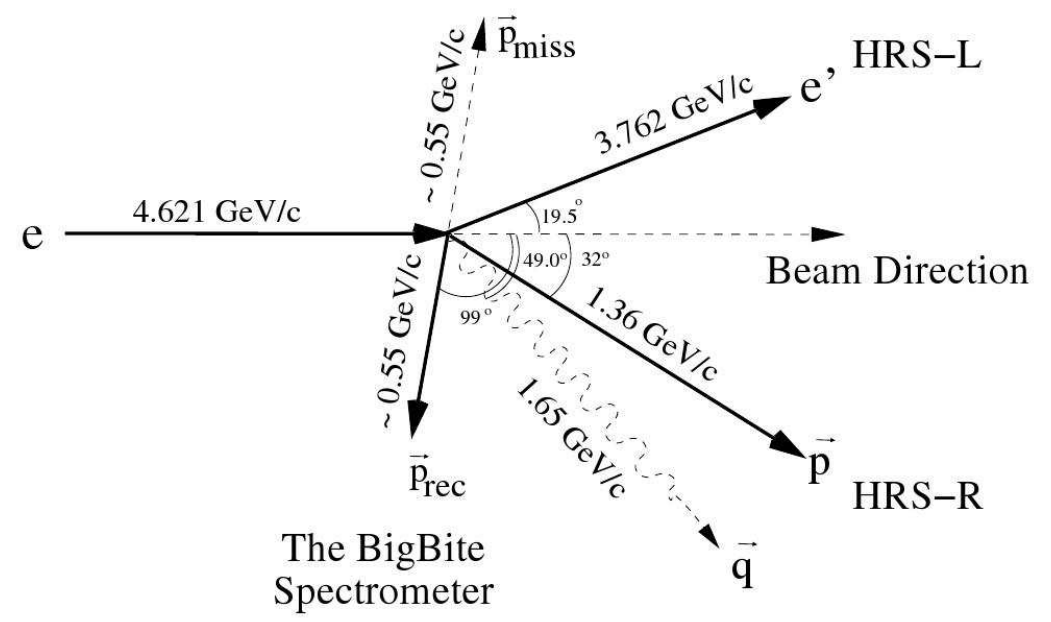

Figure 2.1: Schematic of the experimental layout for E01015 in Hall A at JLab.

\subsection{Thomas Jefferson National Accelerator Facil- ity}

The Thomas Jefferson National Accelerator Facility (known as Jefferson Lab or JLab) is located in Newport News, Virginia, USA. It was jointly commissioned and designed by the U.S. Department of Energy and the Southern Universities Research Association (SURA). The main research goal of the laboratory is to investigate and further the understanding of hadronic structure and nuclear properties at the sub-nuleonic level at energies up to $6 \mathrm{GeV}$. Since the first data was taken in 1995 it has established itself as the world's leading intermediate energy electron facility and it will remain at the forefront with the proposed 12 $\mathrm{GeV}$ energy upgrade, a new experimental hall and upgrade of the three existing experimental halls (Halls A, B and C). 
The Continuous Electron Beam Accelerator Facility (CEBAF) at JLab [69] delivers an continuous-wave electron beam of high quality to the three experimental halls simultaneously, see fig. 2.2. The electrons are injected at energies of a few $\mathrm{MeV}$ into one of the superconducting linear accelerators (known as linacs), which is linked to a similar anti-parallel linac by nine recirculating beam lines in a race-track configuration. The electron beam can be recirculated up to five times, gaining up to $1.2 \mathrm{GeV}$ energy each pass and therefore reaching a maximum energy of $\sim 6 \mathrm{GeV}$. Each hall can independently receive electron bunches which a have undergone a different number of passes and hence have different energies.

The polarised electron beam is created by three $499 \mathrm{MHz}$ radio frequency $(\mathrm{RF})$ gain-switched diode lasers shining on a strained gallium arsenide (GaAs) photocathode under high vacuum. Each laser beam incident on the photo-cathode produces an electron beam at the source. By separating the lasers by $120^{\circ}$ of R.F. phase the three beams may be interlaced and accelerated in a common 1497 $\mathrm{MHz}$ field. R.F. separators at the hall entrances allow each hall to accept every third beam bucket, which can have different current and polarisation states.

There are 20 cryomodules in each linac and 8 Niobium superconducting radio frequency (S.R.F) cavities in each cryomodule. The S.R.F cavities are cooled to $2 \mathrm{~K}$ with liquid helium. The use of superconducting accelerator technology means that the CEBAF machine can support much higher power densities than traditional accelerators since no energy is dissipated through ohmic heat transfer. This allows the machine to run continuously in continuous-wave mode with high voltage gradients. Room temperature linacs have to cycle the beam on/off or use much lower voltage gradients to avoid meltdown. The electromagnetic field in each cavity oscillates in phase with the passing electron producing a negative charge behind the electron and a positive charge in front of the electron, effectively pushing the electron from behind and also pulling it forward at the same time. This results in the increase in electron momentum by $\sim 0.6 \mathrm{GeV}$ every time it passes through one linac. Five separate recirculation arcs transport beam between the linacs allow the beam to recirculate up to five times and extraction may be performed after any recirculation. Hall $\mathrm{A}$ and $\mathrm{C}$ can receive currents of up to $120 \mu A$ whereas Hall B is limited to $\sim 1 n A$ due to the large acceptance CLAS detector system, which is not able to handle large luminosities. 


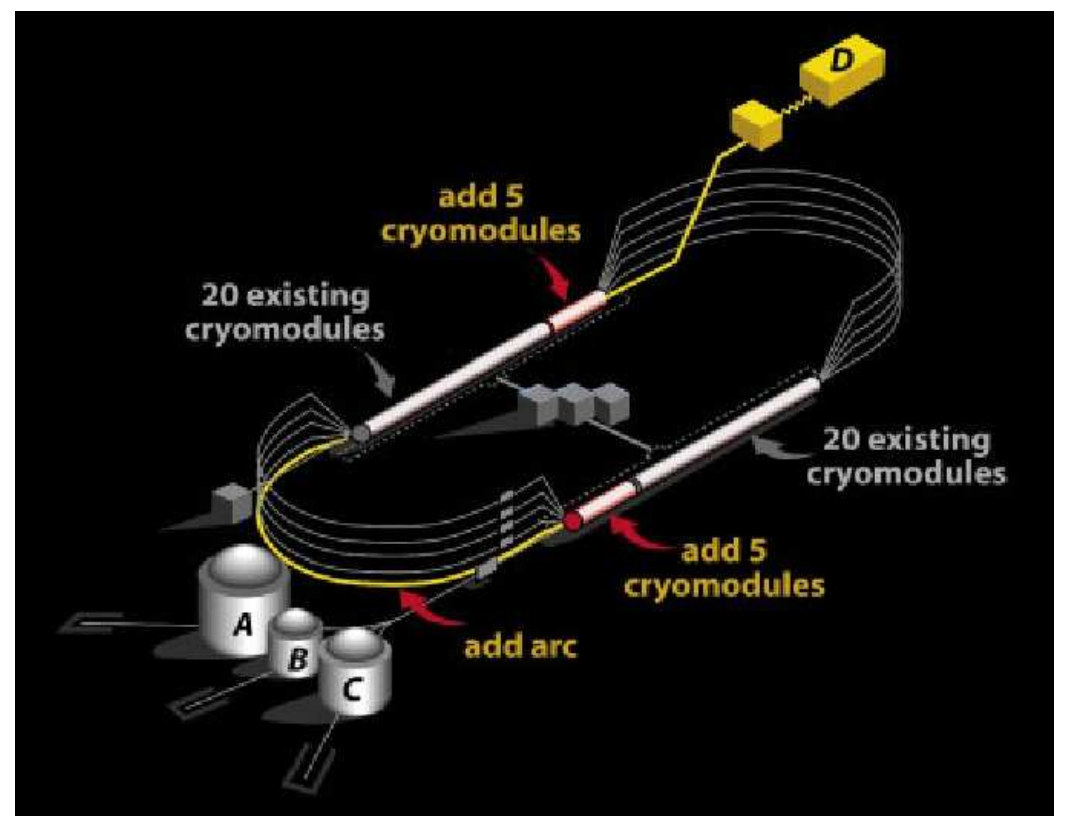

Figure 2.2: Schematic of CEBAF.

\subsection{Experimental Hall A}

Hall $\mathrm{A}$ is the largest experimental hall at JLab and was designed for experiments that require high luminosity and good momentum resolution, see figure 2.3. It has two nominally identical High Resolution Spectrometers (HRS) located either side of the beamline, which can be rotated about the target over a wide range of angles. Many experiments have also required additional detectors and this experiment was the first to use the BigBite large acceptance spectrometer. Various beamline devices between the beam switch-yard and the target were used to monitor and measure the electron beam conditions. A more complete description can be found in the reference [10]. 


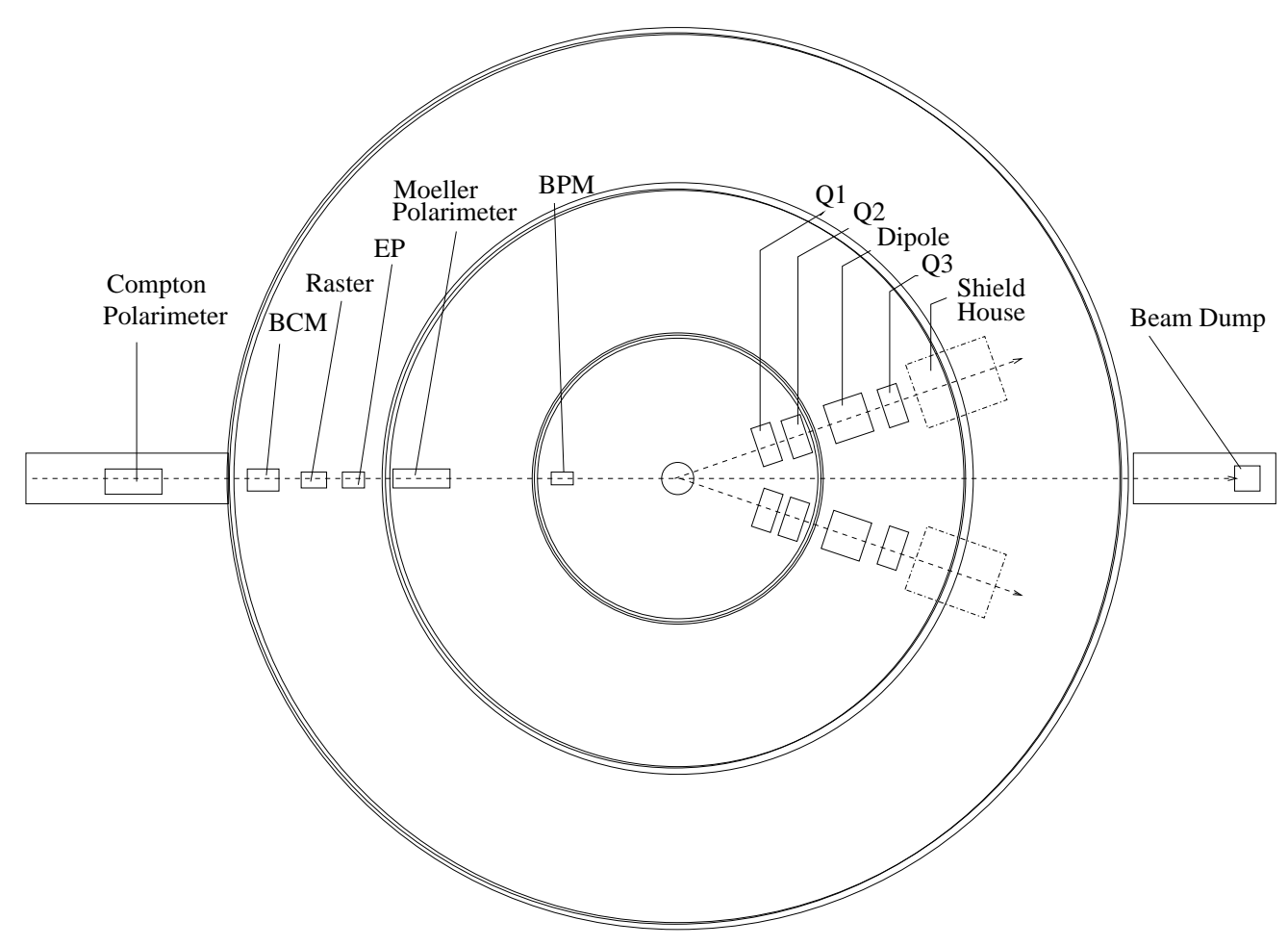

Figure 2.3: Schematic of Hall A.

\section{Experimental Apparatus}

\subsubsection{Electron Beam Monitors}

The beam transport system to the experimental target contains several monitoring devices to measure its relevant properties. The beam properties that need to be monitored and controlled are listed in table 2.1. There are two or more alternative methods for measuring each beam property and this allows each detector's performance to be independently verified and also means there is backup instrumentation should one piece of apparatus fail. The beam parameters are measured to a higher precision than is required by approved experiments.

\begin{tabular}{|c|c|c|c|}
\hline Beam Parameter & Beamline Devices & Measurement Range & Accuracy \\
\hline \hline Position (at target) & BPM/Harp & - & $140 \mu m$ \\
\hline Direction (at target) & BPM/Harp & - & $30 \mu r$ \\
\hline Current & BCM & $40 \mu \mathrm{A}$ & $\leq 2 \times 10^{-3}$ \\
\hline Energy & ARC/ep & $3.471 \mathrm{GeV}$ & $2 \times 10^{-4}$ \\
\hline Polarisation & Moeller & 0.766 & 0.026 \\
\hline
\end{tabular}

Table 2.1: Beamline device properties. The Moeller polarimeter was not used in this experiment. 
The beam position and direction at the target is determined by using two Beam Position Monitors (BPM) which are located $7 m$ and $1 m$ upstream of the target for both time averaged and "event-by-event basis". As the electron passes each BPM its position is reconstructed using induced signals in four antennae that surround the beam in a diamond configuration. Wire "harp" scanners, which are surveyed regularly with respect to the Hall A coordinates, are located in the beamline adjacent to each BPM and used to cross-calibrate the BPMs. A wire scanner is a set of three evenly spaced wires. During calibration the wires are moved across the beam and when a wire is struck by the beam, the current generated allows the position of the beam to be determined.

The Hall A Beam Current Monitors (BCM) consist of two cylindrical resonant cavities which are tuned to the radio frequency of the electron beam and are located about $24 \mathrm{~m}$ upstream of the target. Inside each cavity is a coaxial loop antenna that detects the resonant magnetic field, which is generated when a beam bunch passes. The signal in the antenna is proportional to the charge in the electron bunch because the magnetic field is proportional to both the electron bunch charge and the antenna signal. A voltage-to-frequency converter and scaler is used to record the signal. The output of each scaler is calibrated every 2-3 months using an Unser monitor, a Parametric Current Transformer which provides an absolute reference. The Unser monitor is calibrated by passing a known current through a wire inside the beampipe, but its output signal drifts on the time-scale of minutes. During typical calibration runs, the current is varied up from zero to its maximum in at least five steps lasting 60 to 90 seconds, so that the drift of the Unser monitor can be taken into account.

The absolute energy of the electrons in the beam is measured using two different methods. Firstly, the Arc method measures the angle the electron is deflected around an arc section of the beamline with a nominal angle of $34.3^{\circ}$. The arc contains eight dipole magnets and is located between the beam switch-yard and the entrance to Hall. The initial strength of the magnetic fields of the dipole magnets are set according to the known momentum of the incoming beam, determined by the number of passes of the electron beam around the accelerator. The angle of deflection $\theta$ is determined by measuring the position of the beam, using wire scanners, before and after the the arc of magnets. $\theta$ is then fine tuned by adjusting the magnetic field strength of the dipoles and the momentum of the electron can then be calculated by relating the deflection to the known arc field integral using $c \frac{\int \vec{B} \cdot \overrightarrow{d l}}{\theta}$, where $c$ is a known constant. The second method is called eP because it measures the reaction $p\left(e, e^{\prime} p\right)$ using a silicon strip detector located 
$17 \mathrm{~m}$ upstream of the target. The electron collides with a stationary proton, inside a polyethylene $\left(\mathrm{CH}_{2}\right)$ target, and the electron and proton scattering angles are measured in the strip detector. Fast timing plastic scintillators, which back the strips, provide precise $\left(e^{\prime} p\right)$ coincidence information. The incoming electron energy can then be determined from a simple analysis of the reaction kinematics.

\subsubsection{Target System}

The target for the main production runs was a $0.25 \mathrm{~mm}{ }^{12} \mathrm{C}$ graphite foil, which was angled at $20^{\circ}$ to the incoming electron so that recoil charged particles would only travel an acceptable small distance through the graphite, to ensure the yield is not compromised too much. The carbon target is mounted inside the scattering chamber on a vertical ladder (see fig. 2.4), which can be moved remotely. This allows other targets mounted on the ladder including other solid, cryogenic liquid and gaseous targets to be switched into the beam. The Hall A cryogenic target system [70] has three independent fluid transfer loops: liquid hydrogen $\left(\mathrm{LH}_{2}\right)$, liquid deuterium $\left(L D_{2}\right)$ and gaseous helium. Liquid hydrogen and deuterium targets of $4 \mathrm{~cm}$ thickness were used during the calibration runs of the new BigBite detector package. The scattering chamber has entrance and exit ports for the beam and $0.38 \mathrm{~mm}$ thick aluminium windows spanning the complete angular range covered by the spectrometers $\left(12.5^{\circ}<\Theta<165^{\circ}\right)$.

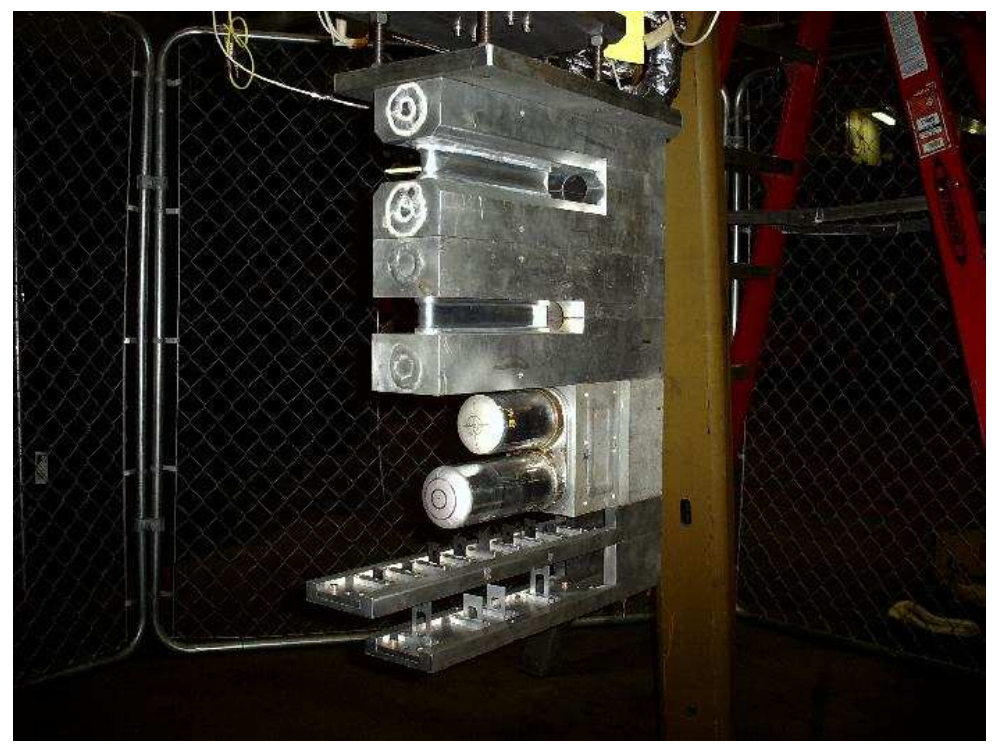

Figure 2.4: Picture of target ladder. Liquid deuterium and hydrogen cells are at the top and some carbon foil targets are at the bottom of the target. 


\subsubsection{High Resolution Spectrometer}

The pair of nominally identical $4 \mathrm{GeV}$ High Resolution Spectrometers (HRS) are the key pieces of apparatus on which most experiments in Hall A are based. Their basic layout is shown in fig 2.5. They provide a very high momentum resolution as well as precise angle and vertex reconstruction. They have moderate acceptance of momentum and sufficient angular acceptance to use extended targets. For this experiment both HRSs were used. The scattered electron was detected in the left HRS and the recoiling proton was detected in the right HRS.

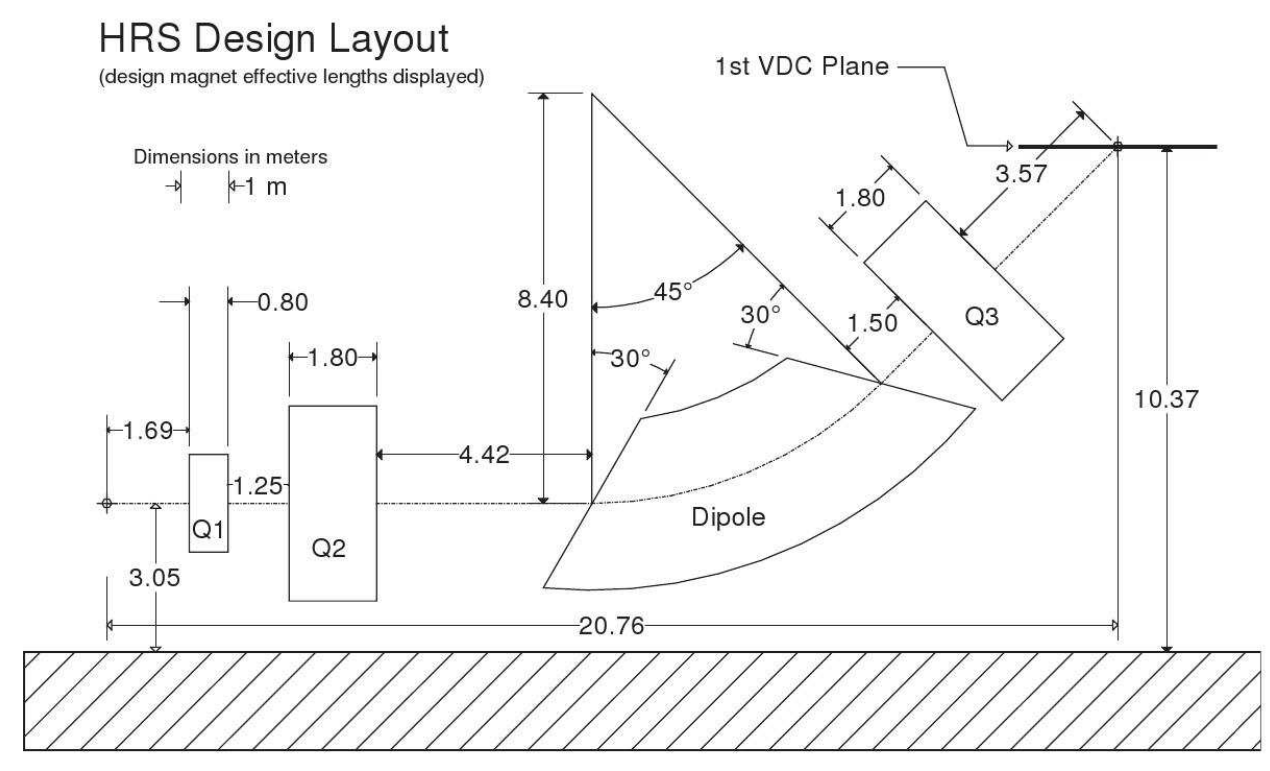

Figure 2.5: Side view of HRS showing the layout of magnet field boundaries.

Each spectrometer uses three superconducting $\cos (2 \theta)$ type quadrupole magnets and one superconducting indexed dipole magnet in the configuration QQDnQ, see fig 2.5. The first quadrupole is vertically focusing and horizontally defocusing (type D), while the second is horizontally focusing and vertically defocusing (type F). Together they combine to produce a parallel beam in the radial plane, which is necessary for the dipole to obtain maximum resolving power for a given bend angle. The quadruples also achieve the desired angular acceptance. To accommodate extended targets two additional quadruples were initially considered, but the design settled on an indexed dipole, which has a non-uniform field component due to the trapezoidal cross section. This makes the radial beam envelope slightly divergent going into the dipole and convergent on the way out. The momentum of charged particles is measured by reconstructing the target position and the bend 
angle from the track detected in the VDC after the Q3 (see fig. 2.6). The dipole has a vertical bend angle of $45^{\circ}$, which provides good momentum resolution. The final quadrupole is vertically focusing and is needed to achieve the desired angular and position resolution. All of the magnet coils are cooled to $4.5 \mathrm{~K}$ by using liquid helium. The central momentum of each spectrometer is set by computer controlled power supplies and NMR probes are used to monitor the dipole field values, while Hall probes are used to monitor the quadrupole field values. The characteristics of the HRS's are summarised in table 2.2.

\begin{tabular}{|c|c|}
\hline Momentum Resolution & $2 \times 10^{-4}$ \\
\hline \hline Momentum Range & $0.3-4.3 \mathrm{GeV} / \mathrm{c}$ \\
\hline Momentum Acceptance & $\pm 5.4 \%$ \\
\hline Angular Acceptance - Horizontal & $\pm 28 \mathrm{mr}$ \\
\hline Angular Acceptance - Vertical & $\pm 60 \mathrm{mr}$ \\
\hline Angular Resolution - Horizontal & $0.6 \mathrm{mr}$ \\
\hline Angular Resolution - Vertical & $2.0 \mathrm{mr}$ \\
\hline
\end{tabular}

Table 2.2: HRS characteristics.

\subsubsection{HRS Focal Plane Detectors}

The detector packages are located at the spectrometer focal planes immediately after the $Q_{3}$ magnets, and are housed within shielding huts. The two HRS detector packages are flexible and can be configured to requirements of individual experiments. The left HRS was setup to detect scattered electrons and the right HRS was setup to detect protons and deuterons (See fig. 2.6).

Left HRS Focal Plane Detector Stack:

- 2 Vertical Drift Chambers (VDC) for precise tracking of electron trajectories.

- 2 Scintillator planes for time of flight and trigger information.

- Lead-glass Cherenkov shower counter and a $\mathrm{CO}_{2}$ gas Cherenkov detector to distinguish between electrons and pions. The Cherenkov detector was removed halfway through the final kinematics run of $0.52 \mathrm{GeV} / \mathrm{c}$ missing momentum to make way for a RICH (Rich Imaging Cherenkov detector) to be used in the next experiment.

The right HRS detector package is similar except it does not have a lead-glass shower counter or a Cherenkov detector but does contain an extra scintillator 
plane. Time of flight and momentum information from the scintillators were sufficient in this experiment to identify protons, deuterons and pions at the present experimental kinematics and therefore the right HRS did not require specific particle identification detectors.

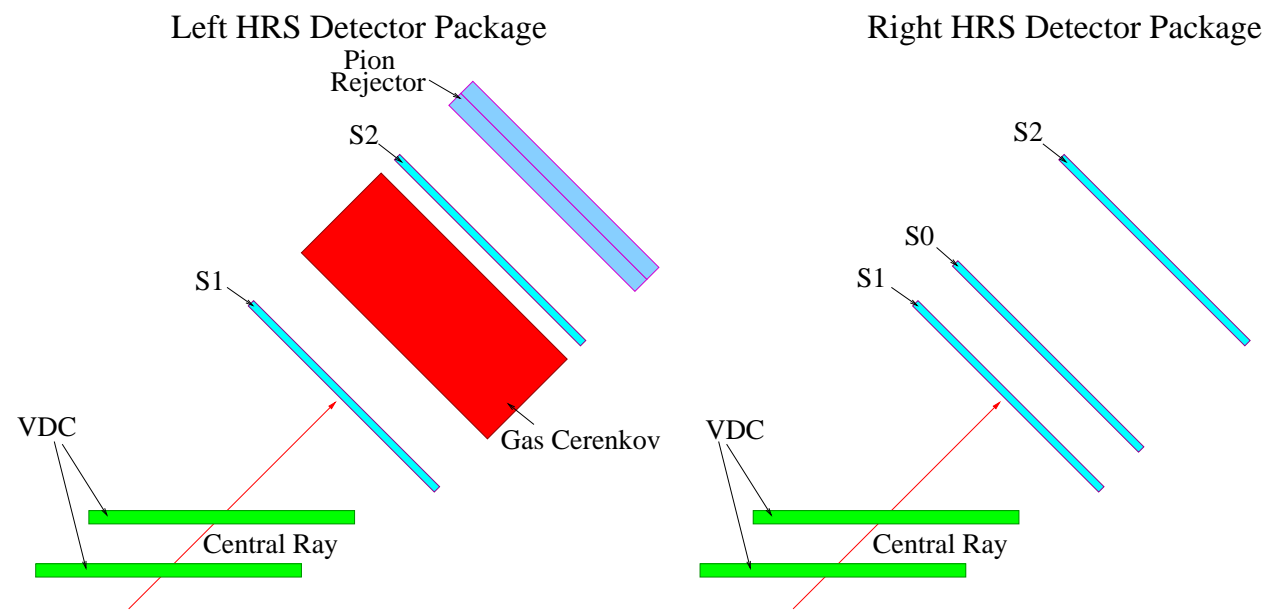

Figure 2.6: Diagram of left and right HRS detector packages.

\section{Vertical Drift Chambers}

A pair of vertical drift chambers (VDC) in each HRS are used to track charged particles with very high precision. The VDC hit coordinates allow the accurate reconstruction of the trajectory and using detailed knowledge of the spectrometer's optical properties, the reaction vertex and the momentum of the track can be reconstructed. The VDCs are located after the $Q_{3}$ magnet and are the first detectors that incoming particles encounter in the HRS. Each VDC is fixed to an aluminium frame, parallel to the Hall floor and slides on rails that are aligned to an accuracy of $100 \mu \mathrm{m}$. The chambers are $240 \mathrm{~cm}$ long, $40 \mathrm{~cm}$ wide and 10 $\mathrm{cm}$ high, and have an active area covering $211.8 \times 28.8 \mathrm{~cm}^{2}$. Each plane has a total of 368 gold-plated tungsten wires spaced $4.24 \mathrm{~mm}$ apart and each VDC chamber contains two planes arranged in a UV configuration, where the wires of each plane are oriented at $90^{\circ}$ to their neighbouring and at $45^{\circ}$ to the central trajectory. Gold plated cathode planes define the electric field of the VDCs and operate at a high voltage of $-4 k V$ when the standard gas mixture of argon (62\%) and ethane $(38 \%)$ is used. This gas mixture is first bubbled through alcohol, to reduce ageing effects on the sense wires, before it flows through the VDCs at a rate of $5 l$ per hour in each of the two chambers. 

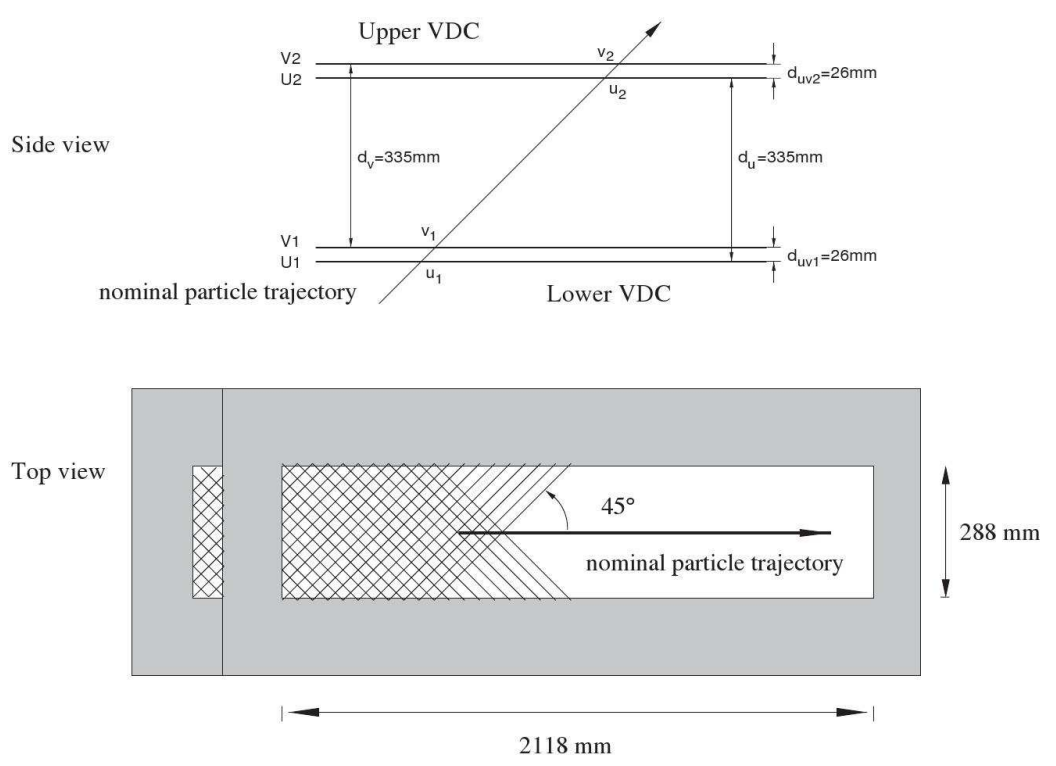

Figure 2.7: The position of the VDCs relative to each other, and to the nominal central trajectory.

Drift chambers detect charged particles by ionisation of the gas atoms, which produce a trail of electrons and ions. The electrons drift with a constant velocity when not close to the sense wire or cathode, due to the uniform and parallel electric field. The electric field becomes stronger as the electron nears the sense wire and accelerates, which causes a secondary electron avalanche. This avalanche produces a negative voltage pulse in the wire which is shaped and amplified. The measured drift time, with respect to the start trigger provided by the S2 scintillator plane, can be used to calculate the perpendicular distance from the particle track using the known drift velocity. In a track, five wires per plane would typically be hit and this information allows the trajectory of the particle to be well mapped out. The position resolution is $\sigma_{x, y} \sim 100 \mu \mathrm{m}$ and the angular resolution is $\sigma_{\theta, \phi} \sim 0.5 \mathrm{mr}$.

\section{The Scintillators Planes}

Two scintillator planes (S1 and S2) in the left HRS and three scintillator planes in the right HRS (S0, S1, S2) were used to provide each HRS's trigger (see section 2.3.1 for trigger details) and to measure the speed of particles $\beta$. The S1 and S2 planes are separated by about $2 m$ and are of the same design in each HRS. The $\mathrm{S} 1$ plane contains 6 thin plastic scintillator paddles of dimensions $0.5 \mathrm{~cm} \mathrm{x}$ $29.3 \mathrm{~cm} \times 36 \mathrm{~cm}$, that overlap by $5 \mathrm{~mm}$ and the $\mathrm{S} 2$ plane contains 16 tightly 
packed paddles of dimensions $0.58 \mathrm{~cm} \times 13.97 \mathrm{~cm} \times 43.18 \mathrm{~cm}$. The S0 plane in the right HRS consists of a single $10 \mathrm{~mm}$ thick paddle and is used for additional trigger information. Each paddle has a photomultiplier tube at each end and time resolution per plane is approximately $0.30 \mathrm{~ns}(\sigma)$.

The measured time-of-flight of each particle between the S1 and S2 planes is used to determine the $\beta$ of the track using the known distance between the scintillator paddles in the different planes with a resolution of $7 \%$. The measured $\beta$ is the main source of particle identification (PID) in the right HRS because no other PID detectors were used. The coincidence time between the triggers of each HRS contains more accurate TOF information because the particles have travelled the long path length from the target to the HRS focal plane $(\sim 25 \mathrm{~m})$. Once the trajectory lengths have been taken into account, a TOF resolution of $\sim 0.5 \mathrm{~ns}$ is obtained.

\section{Gas Cherenkov Detector}

The gas Cherenkov detector was used to distinguish between electrons and pions in the left HRS [71]. Cherenkov radiation is produced by charged particles which exceed the speed of light of the detector medium, $\beta_{\text {Particle }}>\beta_{\text {Medium }}=\frac{1}{n}$, where the refractive index $n$ defines the medium's speed of light. Cherenkov light is produced in a narrow light cone of angle $\theta_{c}=\cos ^{-1} \frac{1}{\beta n}$ and is detected in a photomultiplier.

The Hall A gas Cherenkov detector is a rectangular tank filled with 1 ATM of $\mathrm{CO}_{2}$ and is mounted between the trigger scintillator planes $\mathrm{S} 1$ and $\mathrm{S} 2$. The $\mathrm{CO}_{2}$ gas has a refractive index of 1.00041 and this means that the detection threshold for electrons is only $0.017 \mathrm{GeV}$, compared to $4.8 \mathrm{GeV}$ for pions. The detection efficiency for electrons is $99 \%$ and the pion rejection efficiency is also $99 \%$. There are ten spherical mirrors with $80 \mathrm{~cm}$ focal length, that reflect focused light to a matching PMT.

\section{Shower Counter}

The left HRS shower counter, also known as the pion rejector, contains two layers of 34 lead glass blocks of dimensions $15 \mathrm{~cm} \times 15 \mathrm{~cm} \times 30 \mathrm{~cm}$ (front) and $15 \mathrm{~cm} \times 15$ $\mathrm{cm} \times 35 \mathrm{~cm}$ (back), see fig. 2.8 for structure. Each block is oriented perpendicular to the particle tracks and has a photomultiplier tube at one end. The shower counter is a small energy calorimeter and is made from lead glass because its 
optical properties and the high $\mathrm{Z}$ of the admixed lead. The higher the $\mathrm{Z}$ of the material the greater probability of the high energy charged particle emitting photons though bremsstrahlung, which will then produce electron-positron pairs. These pairs in turn will emit radiation and a cascade of electromagnetic showers will be generated until the momenta of the particles drop to about $100 \mathrm{KeV}$ and then energy is deposited by ionisation and excitation processes. An electron has an absorption length that is much shorter than a pion. This means that most of the electron's energy is deposited in the shower detector, while only a fraction of the pion's energy is absorbed. The combination of the shower counter and gas Cherenkov provides a pion to electron suppression of a factor of $2 \times 10^{5}$ and electron detection efficiency greater than $98 \%$.

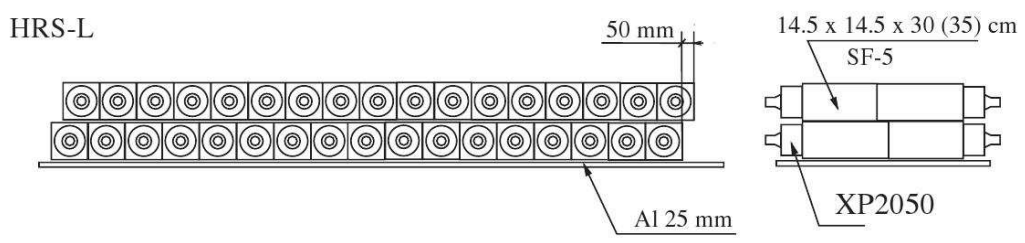

Figure 2.8: Diagram of the left HRS shower counter. Particles enter form the bottom of the figure.

\subsubsection{BigBite Spectrometer}

The BigBite spectrometer was used to detect recoiling protons and deuterons in the ${ }^{12} C\left(e, e^{\prime} p p\right)$ or ${ }^{12} C\left(e, e^{\prime} p d\right)$ events respectively. BigBite consists of a large acceptance non-focusing dipole magnet and detector package (see fig. 2.9). The spectrometer was oriented at an angle of $99^{\circ}$ on the right side of the beam and placed $1.1 \mathrm{~m}$ back from the target. The resulting angular acceptance is about $96 \mathrm{msr}$ and the nominal momentum acceptance range is from $0.25 \mathrm{GeV} / \mathrm{c}$ to 0.9 $\mathrm{GeV} / \mathrm{c}$.

The E01015 experiment was the first in Hall A to use the BigBite magnet and its new detector package that was constructed for this experiment. The BigBite (BB) magnet was originally constructed for use in the Internal Target Facility of the AmPS ring at NIKHEF [72,73] (National Institute for Nuclear and High Energy Physics) located in Amsterdam, Netherlands. This magnet was purchased by JLab and University of Virginia (UVA) to enable three arm experiments with two high resolution arms and a large solid angle arm. During this experiment, the BB magnet provided a field of $0.93 \mathrm{~T}$ at a current of $518 \mathrm{~A}$. 


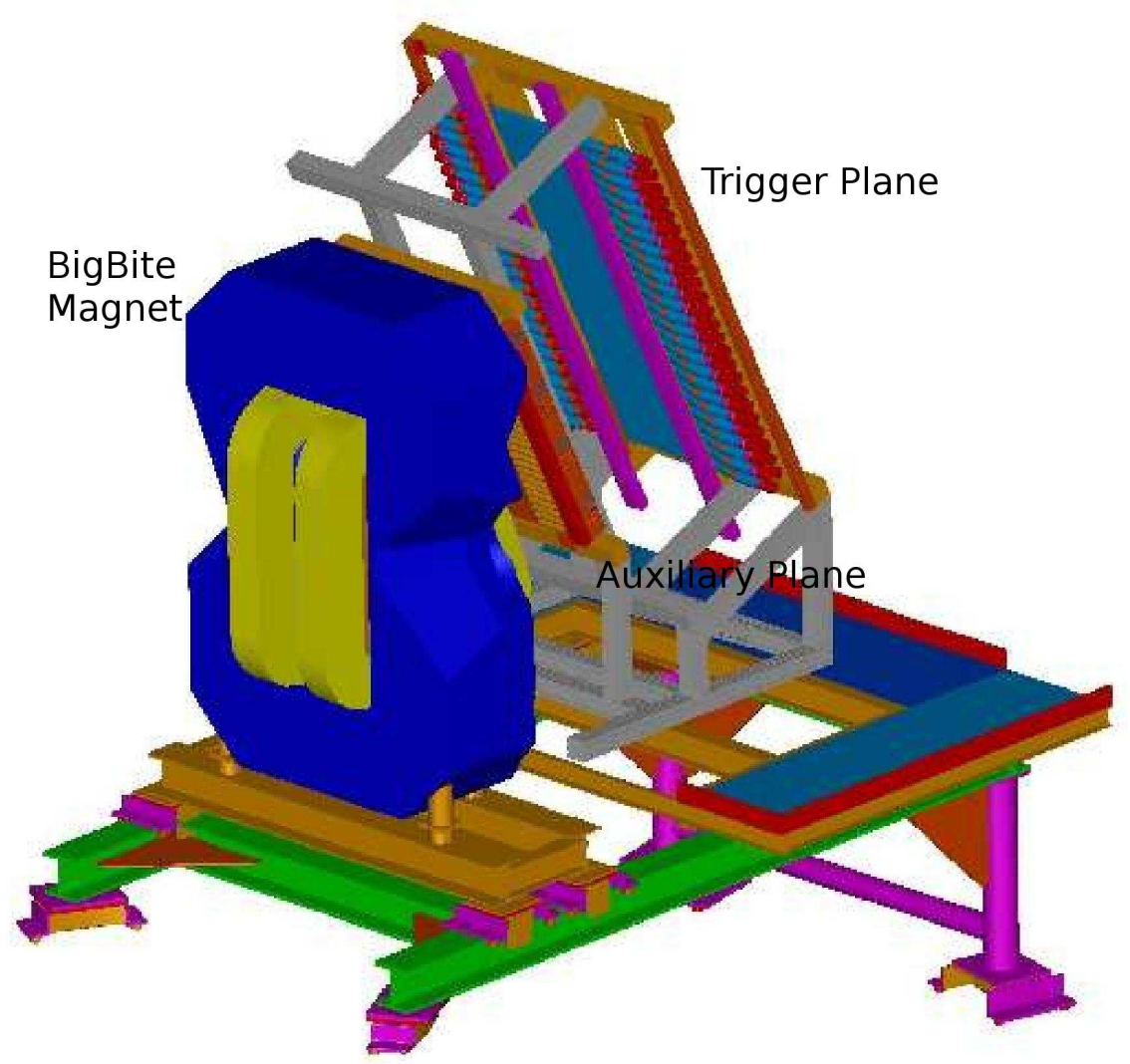

Figure 2.9: CAD drawing of the BigBite magnet and its detector package consisting of the auxiliary and trigger scintillator planes.

\section{BigBite Detector Package}

The detector package was composed of three planes of plastic scintillators segmented in the dispersive direction (see fig. 2.10). The first plane, called the auxiliary plane, was located just after the exit of the BigBite magnet and parallel to its magnetic field boundary. There are 56 narrow scintillator bars in the auxiliary plane of dimensions $350 \mathrm{~mm} \times 25 \mathrm{~mm} \times 2.5 \mathrm{~mm}$ with a single PMT at one end. The other two planes are collectively known as the trigger plane and they are mounted together $1 \mathrm{~m}$ downstream of the first plane. The first trigger plane is called the $\mathrm{dE}$ plane and it consists of 24 thin plastic scintillator bars of dimensions $500 \mathrm{~mm} \times 86 \mathrm{~mm} \times 3.0 \mathrm{~mm}$. The second trigger plane is called the E plane and it also contains 24 scintillators of the same area but they are at the thicker width of $30 \mathrm{~mm}$. The scintillator bars in these two planes are offset by the height of half a bar, effectively improving the vertical position resolution by a factor of two to $43 \mathrm{~mm}$. The trigger plane scintillator bars were each viewed 
by two photomultiplier tubes, one at each end.

Particle identification is determined by using the energy deposited in the $\mathrm{dE}$ and E bars and by the TOF between the auxiliary and trigger planes and the TOF from the target to the trigger plane. The momentum of the particle track and its out-of-plane angle is calculated by using the vertical angle of the particle through the scintillator planes and tracking the particle path back through the magnet to the target. The hit position along the bar is obtained from the time difference of the photomultiplier tubes of the bar hit. Since the dipole magnet is non-focusing, the hit position along the bar corresponds to the in-plane angle of the scattering of the recoiling particle. Using these measured variables the charged particles in BigBite can be identified and a Lorentz vector of particle track in the target can be formed. More details of how BigBite events are analysed are in section 3.4.6.

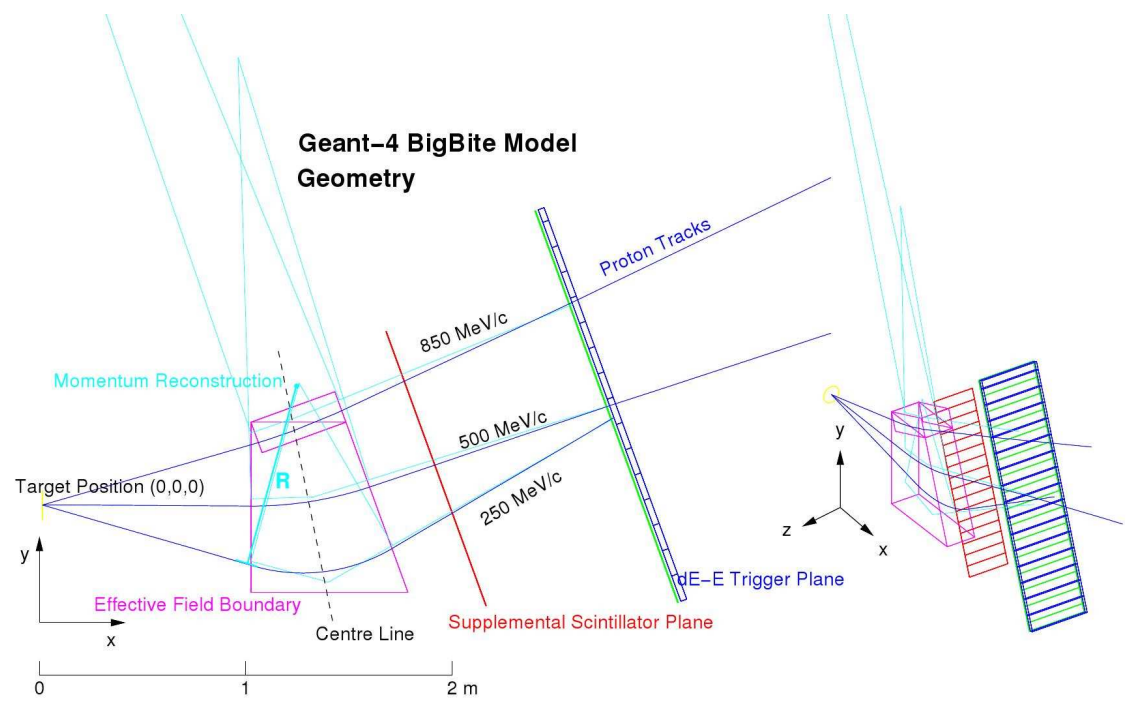

Figure 2.10: Diagram of BigBite produced from Geant 4 simulation [7,8]. It shows three particle tracks of different momentum and out-of-plane angle including the central ray of $500 \mathrm{MeV} / \mathrm{c}$.

\subsubsection{Neutron Detector}

A neutron detector was placed about $6 m$ directly behind the BigBite spectrometer to detect recoiling neutrons from ${ }^{12} C\left(e, e^{\prime} p n\right)$ events that have passed directly through the BigBite magnet, undeviated by the magnetic field. The neutron detector was comprised of a veto plane followed by four scintillator planes (see fig. 2.11).

The veto plane has 32 rows with two thin veto bars each that overlap by 30 $\mathrm{cm}$. The dimensions of the veto bars are $70 \mathrm{~cm} \times 11 \mathrm{~cm} \times 2 \mathrm{~cm}$ and each has a 
single photomultiplier tube (PMT). The four scintillator planes have bars with a length of $100 \mathrm{~cm}$ and width of $10 \mathrm{~cm}$, but they vary in height because they were sourced from different universities and facilities. The number and height of the bars in the planes were: plane 1 had 30 bars of $10 \mathrm{~cm}$ height, plane 2 had 24 bars of $12.5 \mathrm{~cm}$ height, plane 3 had 8 bars of $12.5 \mathrm{~cm}$ height plus 2 bars of 10 $\mathrm{cm}$ in the middle and plane 4 had 12 bars of $25 \mathrm{~cm}$ height. Each scintillator in these planes were viewed by two PMTs allowing the hit position along the bar to be calculated from the time difference from the PMT signals. The physical size of the neutron detector is $317 \mathrm{~cm}$ in height by $59 \mathrm{~cm}$ in width and $100 \mathrm{~cm}$ in thickness, taking into account the spacing of the scintillators and not including the extra width of the PMTs. These dimensions of the neutron detector and its placing were specified so that the solid angle would match that of the acceptance of the BigBite magnet. The in-plane acceptance angle was about $\pm 5^{\circ}$ and the out-of-plane acceptance was about $\pm 15^{\circ}$.

The veto plane is necessary for detecting charged particles entering the front of the detector, thus providing a veto for hits in the remaining scintillator planes that are not neutrons or photons which can be separated using TOF. The neutrons pass through the veto plane without leaving any detectable interaction and will only be detected if they undergo a hadronic reaction such as knocking out a proton from a nucleus in one of the scintillators. The understanding of this probability is crucial for cross-section determination and has been calculated to be $16.5 \pm 2.8 \%$ from the detailed study of ${ }^{2} H\left(e, e^{\prime} p\right) n$ reaction [67] done during the commissioning of the detector. The main method of counting the number of neutron hits in the ${ }^{12} C\left(e, e^{\prime} p n\right)$ reaction was to count the number events within neutron peak of the TOF spectrum of the neutron detector in coincidence with good ${ }^{12} C\left(e, e^{\prime} p\right)$ events. 


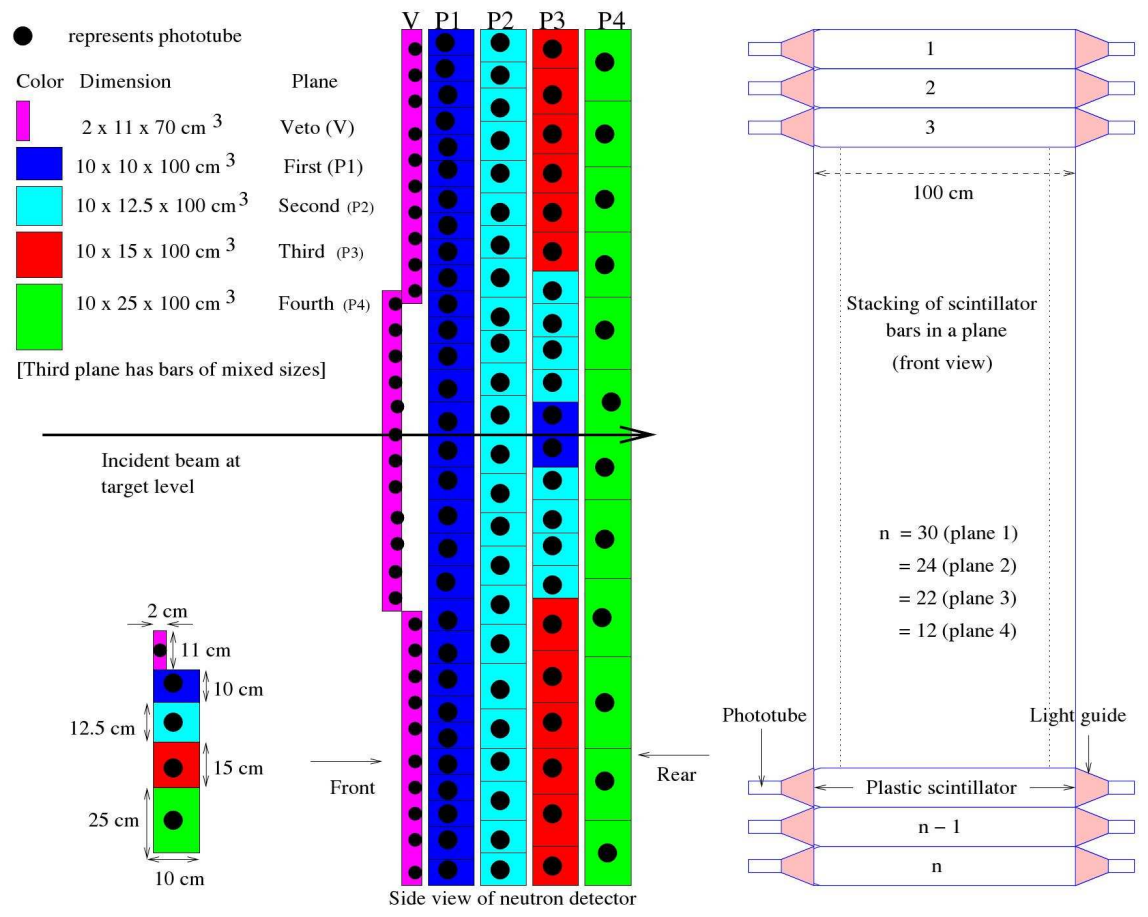

Figure 2.11: Scintillator and veto bar configuration of the Neutron Detector.

\subsection{Data Acquisition}

\subsubsection{Trigger Scheme}

An event is recorded by the data acquisition system if it passes the logic circuit defined by the trigger electronics. The Photomultiplier (PMT) signals from the scintillator planes in the both HRSs, BigBite and the Neutron detector are used to trigger the start of data acquisition and to define the type of event. Scintillators are commonly used as triggers for composite detector systems because their response time is extremely fast, meaning a good timing resolution is possible.

The trigger generated from a hit in a scintillator plane is formed from the logical OR of the all signals of each bar. The individual bar signal requires a coincidence signal between both PMTs. The list of triggers and their conditions used in the experiment is as follows.

- T1: Left HRS scintillator planes S1 and S2 coincident in timing window of 100 ns. This trigger signifies that there was an event in the left HRS. This trigger had a final prescale factor (the number of events for one event recorded) of 176 and a prescale/real trigger rate of 20.9/3682.8 Hz. 
- T2: Right HRS scintillator plane S1 or S2 and scintillator plane S0 coincident have signals. This trigger had a final prescale factor of 12 and a prescale/real trigger rate of $5.1 / 61.7 \mathrm{~Hz}$.

- T3: Right HRS scintillator planes S1 and S2 coincident in timing window of 50 ns. This trigger signifies that there was an event in the right HRS and it also opens the ADC gates of the BigBite and Neutron detectors, as well as providing the common start for the BigBite TDCs and common stop for the neutron detector TDCs. This trigger had a final prescale factor of 36 and a prescale/real trigger rate of $18.8 / 678.5 \mathrm{~Hz}$.

- T4: Left HRS scintillator plane S1 or S2 and gas Cherenkov have signals. This trigger had a final prescale factor of 80 and a prescale/real trigger rate of $3.3 / 266.3 \mathrm{~Hz}$.

- T5: Logical AND of triggers T1 and T3 in timing timing window of about 100 ns. This trigger effectively arrives earlier than other triggers because the others are delayed by 60 to $80 \mathrm{~ns}$ relatively. This was done to prioritise the most important trigger, a coincident event in both HRS. This trigger had a final prescale factor of 1 and a prescale/real trigger rate of $0.9 / 0.9$ $\mathrm{Hz}$.

- T6: Trigger signal from the BigBite trigger plane. Not recorded into the data stream due to the very high rate of this trigger (approx $30 \times \mathrm{T} 1$ ). This trigger had a final prescale factor of 30400 and a prescale/real trigger rate of $3.9 / 117071.3 \mathrm{~Hz}$.

- T7: Trigger signal from the Neutron Detector. Not recorded into the data stream due to the very high rate of this trigger (approx $800 \times \mathrm{T} 1$ ). This trigger had a final prescale factor of 3040000 and a trigger prescale/real rate of $0.0 / 3059768.8 \mathrm{~Hz}$.

T1, T3 and T5 are taken to be good events, while the T2 and T4 were used to study scintillator inefficiencies. T6 and T7 were used to check that the timing of BigBite and the Neutron detector were reasonably correct. All the triggers were sent to scalers to be recorded and also to the Trigger Supervisor (TS). The TS prescales the triggers and synchronises the readout crates. It also administers the dead time logic of the entire system which was kept below $20 \%$ with an event rate below $500 \mathrm{~Hz}$. The acquisition rate and the dead time are proportional to the beam current, which was limited to $20 \mu \mathrm{A}$. All the trigger types were given 
large prescale values during production running except for the most important T5 trigger. The prescale values were varied during calibration and commissioning runs at the start of the running period to test the new equipment. The prescale rates given in the list above were for a production run with a $20 \mu \mathrm{A}$ current.

\subsubsection{Electronics}

The output of every detector during this experiment was stored as raw data read out from the ADC and TDC channels. Software called CODA (CEBAF Online Data Acquisition) [74] controlled the data acquisition of these individual channels into the data-stream, ultimately to be saved as data files in JLab mass storage system. For each HRS, the data acquisition electronics system was composed of one VME crate and one Fastbus crate and they were all located in the spectrometer shielding house. All the ADCs and TDCs for both HRSs were fastbus based and the scalers were fed into the VME crate. The BigBite and the Neutron Detector data acquisition electronics (see fig 2.12) were located on the right hand side of Hall A and were shielded from radiation by a wall of concrete blocks. The triggers from both HRSs and the BigBite and Neutron Detector were all sent to one trigger supervisor located in the BigBite electronics rack. 

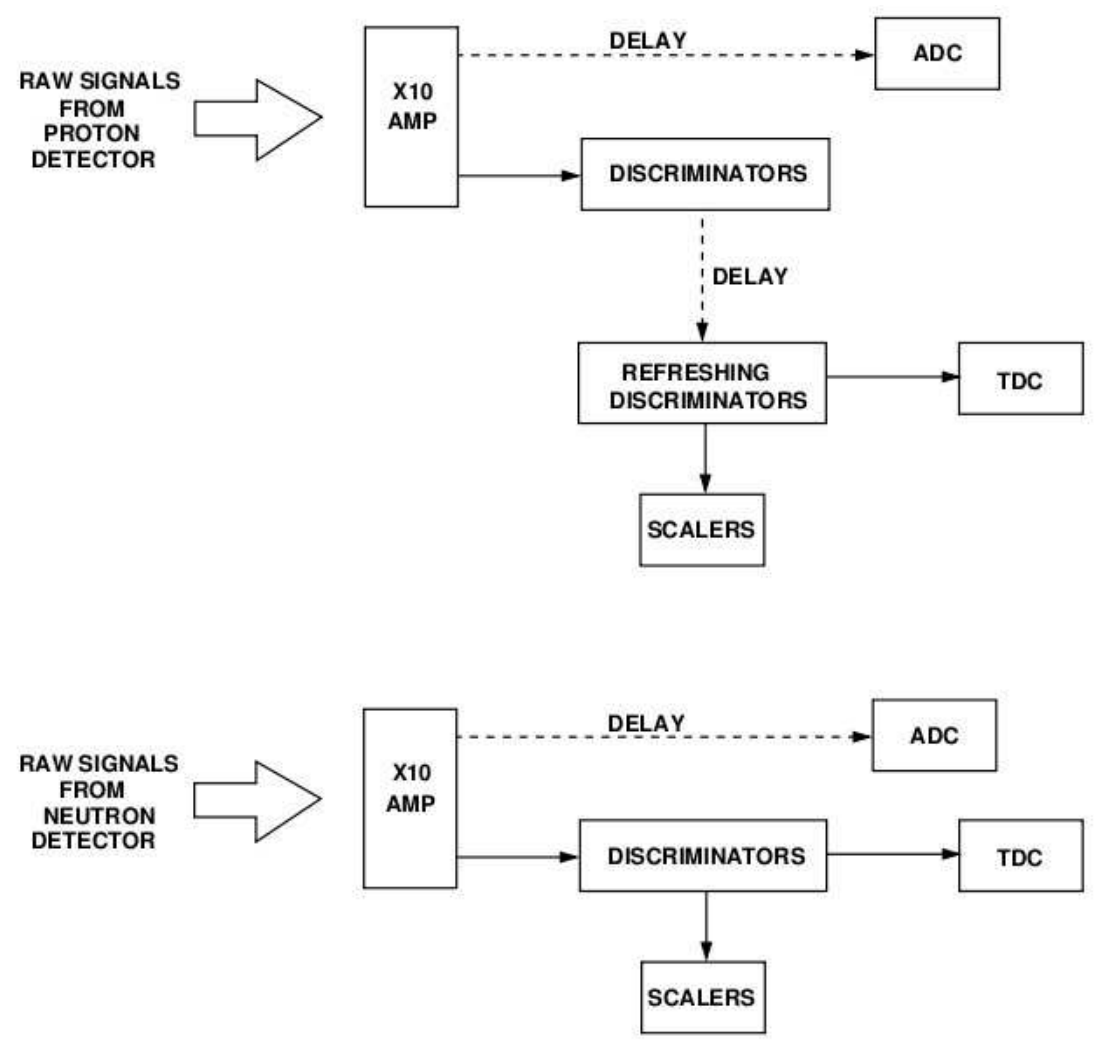

Figure 2.12: Data acquisition packages of BigBite and the Neutron Detector. Picture courtesy of Peter Monaghan [9].

The data acquisition software CODA was run on a computer in the counting house of Hall A. Fastbus interfaces called ReadOut Controllers (ROC) control the ADC and TDC modules in the fastbus and VME crates. The trigger supervisor controls the run state and generates triggers that cause the ROC to be read out. An event builder collects the readout from the ROCs and organises the necessary CODA header information to build an event. These events are analysed by the Hall A Analyzer [75], which is based on ROOT [76]. ROOT is a object oriented frame work for large scale physics analysis written in $\mathrm{C}++$ that for example can be used for manipulating data, creating histograms, performing statistical analysis and saving the experimental data into organised and compressed data files. The Analyzer saves the analysed data into root files on a computer in the Hall A counting house and later transfers them to the JLab mass storage system. 


\section{Chapter 3}

\section{Data Analysis: Event Selection}

The analysis of the ${ }^{12} C\left(e, e^{\prime} p d\right)$ reaction consists of two main parts: the selection of events of interest and the extraction of a yield. After the yield was obtained the cross section was estimated using acceptance corrections obtained from Monte Carlo simulations. This chapter explains the how events are selected and the unfolding of cross-sections from the experimental data.

\subsection{Overview}

The analysis was performed in two stages. The first used the "Hall A Analyzer" software package [75], which is written in $\mathrm{C}++$ and based on ROOT [76]. It converted the data files filled with raw digital TDC and ADC information into observable physical quantities. Conversion parameters were obtained from calibration analysis of each sub-detector and fed into the Analyzer programme. Calibrations of the HRS detectors were performed by other members of the collaboration $[9,67,68]$.

Output from the Hall A Analyzer was further analysed by a ROOT based $\mathrm{C}++$ code called Fireroot. The purpose of this code was to correlate information from the various components of the experiment, the two HRS's and BigBite. It was also used to augment the Hall A Analyzer implementation of BigBite, which was inadequate for the present analysis as it assumes only protons are of interest in BigBite and neglects the search for and tracking of deuterons.

Most of the data analysed with the Hall A Analyzer has T5 (Left + Right HRS, see section 2.3.1) trigger condition cuts applied to select only $\left(e, e^{\prime} p X\right)$ events that may be of interest. Data for calibration purposes were collected using different trigger conditions. The cuts discussed later in this chapter are added at 
the formation of the histograms in macros.

\subsection{Electron Analysis}

The analysis of the scattered electron in the left HRS consists of three parts: track reconstruction, reaction vertex reconstruction and particle identification. Track reconstruction consists of analysing the drift times associated with wire hits along particle tracks as they pass through each Vertical Drift Chamber (VDC) and then combining together the information from the two sets of VDC pairs to form global tracks. The initial momentum vector at the reaction vertex in the target is reconstructed by applying very accurately known transport matrices to the VDC track. Particles are identified by their response in the shower counter and Cherenkov detectors, which form part of the left HRS detector stack (see section 2.2.4). The track and target reconstruction for the right HRS is very similar to the left HRS, only the particle identification methods are different.

\subsubsection{VDC Track Reconstruction}

The time taken for electrons, produced by ionisation along the track of the scattered electron, to drift to the anode wires in each VDC is measured and converted into the perpendicular distance from the wire. This drift time is obtained from the wire TDC using its conversion gain, taking into account the time offset that arises from different propagation delays through the electronics and the time of flight between the wire and the trigger scintillator plane S1. The distance of the scattered electron to the wires is calculated using $d=v_{d} t_{d}\left(1+a_{2} / a_{1}\right)$, where $v_{d}$ is the drift velocity and $t_{d}$ is the drift time. $a_{1}$ and $a_{2}$ are polynomials in $\tan \theta$ and are used to describe the non-linear relationship between drift time and drift distance due to the acceleration of the electron caused by the increase in electric field strength as it approaches the wire [77]. Subsequently, adjacent hit wires in each plane are grouped into local clusters, the pivot wire is determined and the trajectory of the track is analysed. The pivot wire is the one with the lowest drift time and therefore the closest to the crossover point of the scattered electron, which is the intercept of the electron's path with the wire plane. A linear fit to the hit positions in each cluster is performed using the the drift distances and the positions of the wires (see fig. 3.1). 


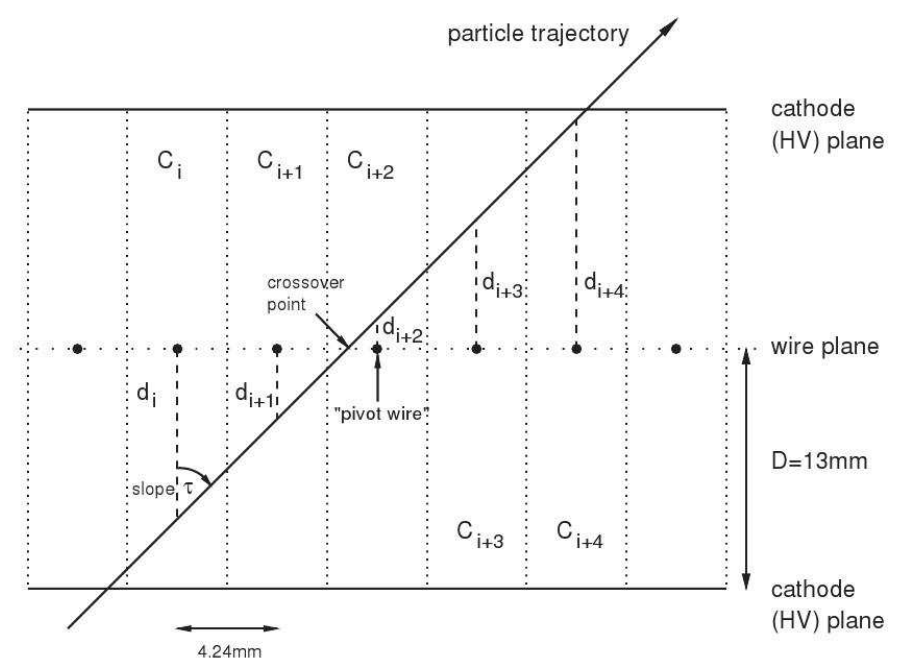

Figure 3.1: A typical cluster of hits in a VDC wire plane. $C_{i}$ denotes the $i$ th wire cell, and $d_{i}$ represents the perpendicular (effective) drift distance within that cell. The $i$ th cell is defined as the region within which ions drift to the $i$ th wire. By symmetry, the cell boundaries lie halfway between wires. The wire spacing is $4.24 \mathrm{~mm}$. The separation between cathode high-voltage and wire planes is approximately $13 \mathrm{~mm}$. The HV planes are held at a potential of approximately $-4 \mathrm{kV}$, while the wires are effectively grounded. Figure taken from [10].

The clusters in the adjacent $\mathrm{U}$ and $\mathrm{V}$ planes are then matched using the timing differences of the pivot wires. Each UV track pair is defined by positions $(U, V)$ and angles $\left(\theta_{U}, \theta_{V}\right)$. $\theta_{U}$ and $\theta_{V}$ are the angles crossing each $\mathrm{U}$ and $\mathrm{V}$ plane respectively (the $\theta$ in figure 3.1). From these $\mathrm{U}$ and $\mathrm{V}$ coordinates, provisional detector coordinates $(x, y, \theta, \phi)$ (see 3.2) are calculated for each UV track pair using simultaneous equations that take advantage of the orthogonal relationship between the two planes to obtain both the dispersive and non-dispersive directions.

The next stage in this process is to create global tracks by combining the tracks from each separate set of UV planes. They are sorted in ascending order of the quality of the match between the individual UV tracks. This is dictated by the distance between the projected track positions and the partner track intercepts on each plane. The detector coordinates are then updated using global $U$ and $\mathrm{V}$ trajectory angles. Finally, the track variables are converted from the local VDC coordinate system to the Transport Coordinate System (TRCS). This is a necessary step in order to obtain the corresponding trajectory at the target using the optical transport properties of the HRS. 


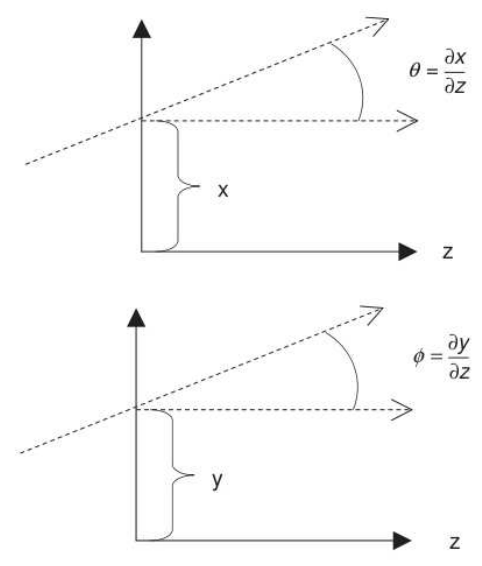

Figure 3.2: Coordinates in the TRANSPORT convention. $z$ is defined by the central or reference trajectory. $x$ and $y$ are perpendicular to $z$, with $x$ in the dispersive plane.

\subsubsection{Target Coordinate Reconstruction}

The trajectory of the track of the scattered electron through the HRS is described by the vector $(\mathrm{t})$ in the transport coordinate system. This vector defines the track relative to the central reference track and it contains five components which are defined as follows.

$$
t=\left[\begin{array}{l}
x \\
\theta \\
y \\
\phi \\
\delta
\end{array}\right]
$$

where:

- $x$ is the displacement in the dispersive (vertical) direction from the central trajectory, expressed in meters.

- $\theta$ is the angle in the same plane relative to the central trajectory $(d x / d z)$, expressed in radians.

- $y$ and $\phi$ are the equivalent to $x$ and $\theta$ in the non-dispersive (transverse) plane.

- $\delta(\triangle p / p)$ is the fractional deviation of momentum of the trajectory from the central trajectory. The central trajectory is the a reference track of a scattered electron with no out-of-plane angle and no in-plane angle relative 
to the spectrometer angle. A specific momentum is required so that it passes through the centre point of all the magnetic fields and detectors. The momentum of the central trajectory is set by the current applied to the magnets in the HRS.

To first order, the track trajectory at the target can be obtained from the track trajectory at the focal plane using a simple transport matrix equation (see below). $x_{t g}$ is known from beam position monitor (BPM) data (see section 2.2.1), therefore only four unknown target parameters are needed in this transport matrix.

$$
\left[\begin{array}{l}
\delta \\
\theta \\
y \\
\phi
\end{array}\right]_{t g}=\left[\begin{array}{cccc}
\langle\delta \mid x\rangle & \langle\delta \mid \theta\rangle & 0 & 0 \\
\langle\theta \mid x\rangle & \langle\theta \mid \theta\rangle & 0 & 0 \\
0 & 0 & \langle y \mid y\rangle & \langle y \mid \phi\rangle \\
0 & 0 & \langle\phi \mid y\rangle & \langle\phi \mid \phi\rangle
\end{array}\right]\left[\begin{array}{c}
x \\
\theta \\
y \\
\phi
\end{array}\right]_{f p}
$$

The actual transport equations used are much more complicated, with the focal plane coordinates replaced by tensors. There are four tensors, labelled $Y_{j k l}$, $T_{j k l}, P_{j k l}$ and $D_{j k l}$, which have elements that are polynomials in $x_{f p}$ up to the fifth order and they link the focal plane and target coordinates according to:

$$
\begin{gathered}
y_{t g t}=\sum_{j, k, l} Y_{j k l} \theta_{f p}^{j} y_{f p}^{k} \phi_{f p}^{l} \\
\theta_{t g t}=\sum_{j, k, l} T_{j k l} \theta_{f p}^{j} y_{f p}^{k} \phi_{f p}^{l} \\
\phi_{t g t}=\sum_{j, k, l} P_{j k l} \theta_{f p}^{j} y_{f p}^{k} \phi_{f p}^{l} \\
\delta=\sum_{j, k, l} D_{j k l} \theta_{f p}^{j} y_{f p}^{k} \phi_{f p}^{l}
\end{gathered}
$$

An optimising procedure is used to obtain these tensors and it also corrects the focal plane coordinates for any detector offsets from the central ray of the spectrometer. This procedure involves detecting electrons scattered quasi-elastically from a set of thin ${ }^{12} C$ foil targets [78], which pass through a 49 hole sieve-slit collimator placed at the entrance to the aperture to the spectrometer to define a set of discrete trajectories. The algorithm, used to optimise the tensor elements, minimises the difference (via $\chi^{2}$ ) in the reconstructed target and sieve-hole positions with the known positions, measured in a survey beforehand. The starting values in the algorithm are usually taken from previous runs at similar settings, 
but the two spectrometers' accuracy are routinely checked. The scattering angle determination and spectrometer offsets are checked using the elastic ${ }^{1} H\left(e, e^{\prime} p\right)$ reaction by comparing the measured scattered electron angle to the calculated scattered electron angle, using the angular relationship between the measured initial and elastically scattered electron energy. The momentum determination and spectrometer constant is checked using the elastically scattered electrons off a thin ${ }^{12} C$ target. For the ground state of ${ }^{12} C$, the momentum of the scattered electron $p$ and the beam energy $E_{i}$ can be related by:

$$
E_{f}=p=\frac{E_{i}-E_{l o s s} 1}{1+2 E_{i} \sin ^{2}(\theta / 2) / M_{t}}-E_{\text {loss } 2}
$$

where $E_{f}$ is the final energy of the scattered electrons, $M_{t}$ is the mass of the target nucleus and $E_{l o s s 1}$ and $E_{l o s s 2}$ is the energy loss before and after scattering. At higher energies (greater than $1.5 \mathrm{GeV}$ ) the cross-section for the ${ }^{12} \mathrm{C}$ elastic scattering becomes too low and missing mass measurements of the $1 \mathrm{p}_{1 / 2}$ state in the ${ }^{12} C\left(e, e^{\prime} p\right)$ reaction were used instead. The target coordinate parameters of electron detected in the left HRS are displayed in fig. 3.3.
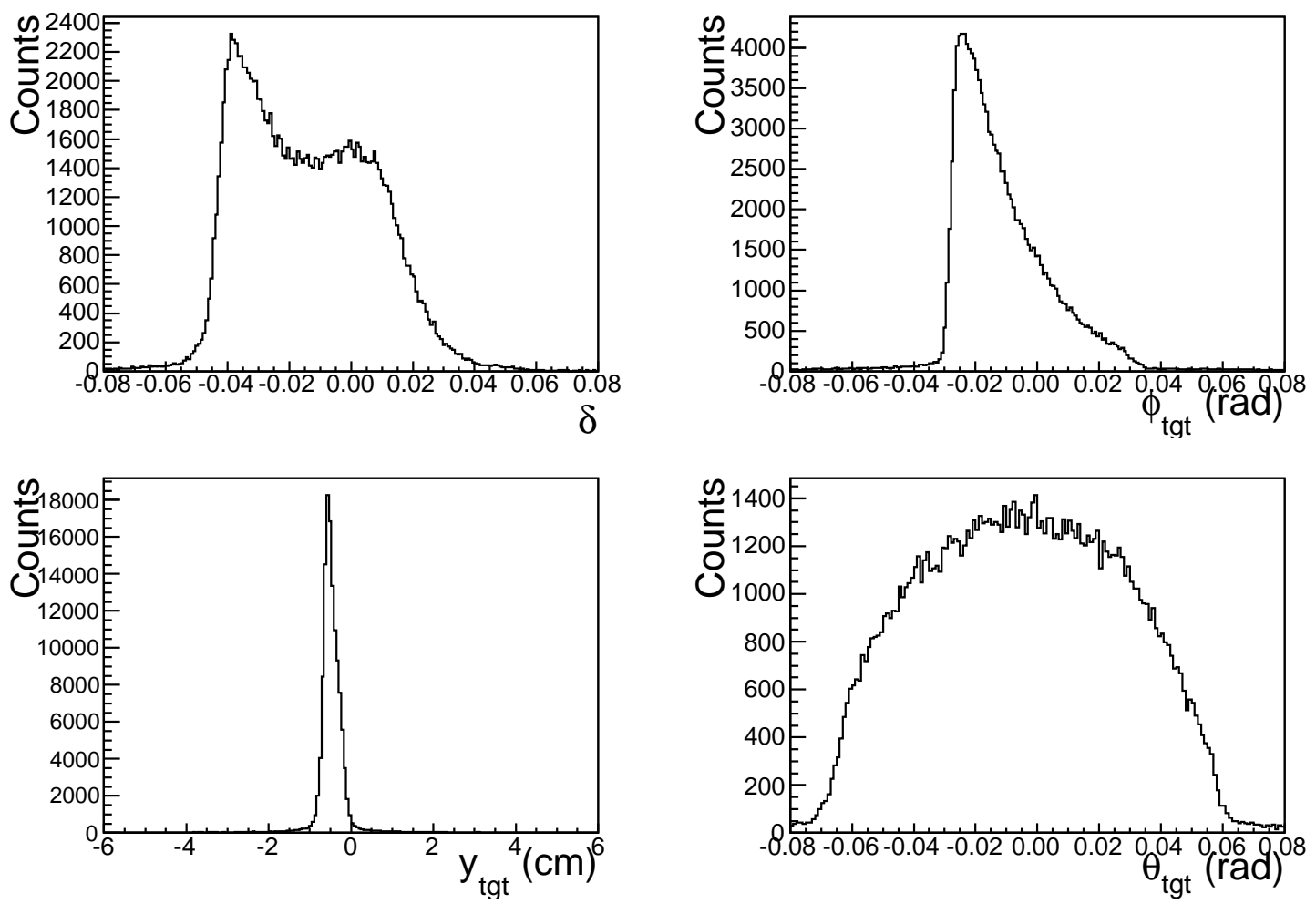

Figure 3.3: Target system electron projection parameters $\delta, \phi_{t g t}, y_{t g t}$ and $\theta_{t g t}$ after optimisation of the HRS optics tensors, using production data taken at the CK1 kinematics $\left({ }^{12} \mathrm{C}\left(e, e^{\prime} p\right)\right.$ missing momentum of $\left.300 \mathrm{MeV} / \mathrm{c}\right)$. 


\subsubsection{Electron Selection}

Before selecting the scattered electron events, data runs were rejected if errors had occurred during the data taking process and good runs of the same kinematics were collected together. Standard left and right HRS cuts were then applied on the trajectory parameters to ensure that the reconstructed tracks were contained within the nominal acceptance of the left HRS, where the momentum reconstruction is reliable. These initial cuts (see table 3.1) were not so stringent as to throw away possible good events in this analysis where the triple coincident cross sections are very small. This process is repeated for analysis of the tracks in the right HRS and the cuts in the table below apply to it unless otherwise noted. Only the "golden track", the track with the smallest chi-squared in combining the U-V hits (see section 3.2.1), was used in this analysis.

The next stage was to use the PID detectors of the left HRS, the shower counter and the $\mathrm{CO}_{2}$ gas Cherenkov detector, to identify electrons and reject pions. The shower counter is also known as the pion rejector as it cleanly separates the pions from the electrons when the energy deposited in the pre-shower versus the shower counter are displayed in a 2D histogram (see fig. 3.4). The graphical cut shown in fig. 3.4 defines the limits of the pion distribution in the bottom left corner. Pion rejection can be further supplemented by also requiring the light deposited in the Cherenkov detector to be above the ADC channel number of 50 . Unfortunately the Cherenkov detector was removed during the experiment, so its data was used mainly for efficiency purposes.

\begin{tabular}{|c|c|c|}
\hline HRS Variable & Description & Cuts \\
\hline \hline$\delta$ & $\triangle p / p$ & $-4.5 \% \leq \delta \leq 4.5 \%$ \\
\hline$y$ & Non-dispersive displacement & $\pm 5 \mathrm{~cm}$ \\
\hline$\theta$ & $\theta=d x / d z$, in vertical direction & $\pm 60 \mathrm{mrad}$ \\
\hline$\phi$ & $\phi=d y / d z$, in transverse plane & $\pm 30 \mathrm{mrad}$ \\
\hline$p$ & Momentum & $p \pm 0.4 \mathrm{GeV} / \mathrm{c}$ \\
\hline$\beta$ & Relativistic Velocity & $0.5 \leq \beta \leq 1.2$ \\
\hline$N_{\text {Tracks }}$ & Number of tracks & $N_{\text {Tracks }}=1$ \\
\hline$x_{s 2}$ & S2 scintillator plane x displacement & $-1.5 \leq x_{s 2} \leq 1.5 \mathrm{~m}$ \\
\hline$R_{z}$ & $\mathrm{z}$ component of reaction vertex & $\pm 0.1 \mathrm{~m}$ \\
\hline
\end{tabular}

Table 3.1: The initial cuts applied to HRS variables. 

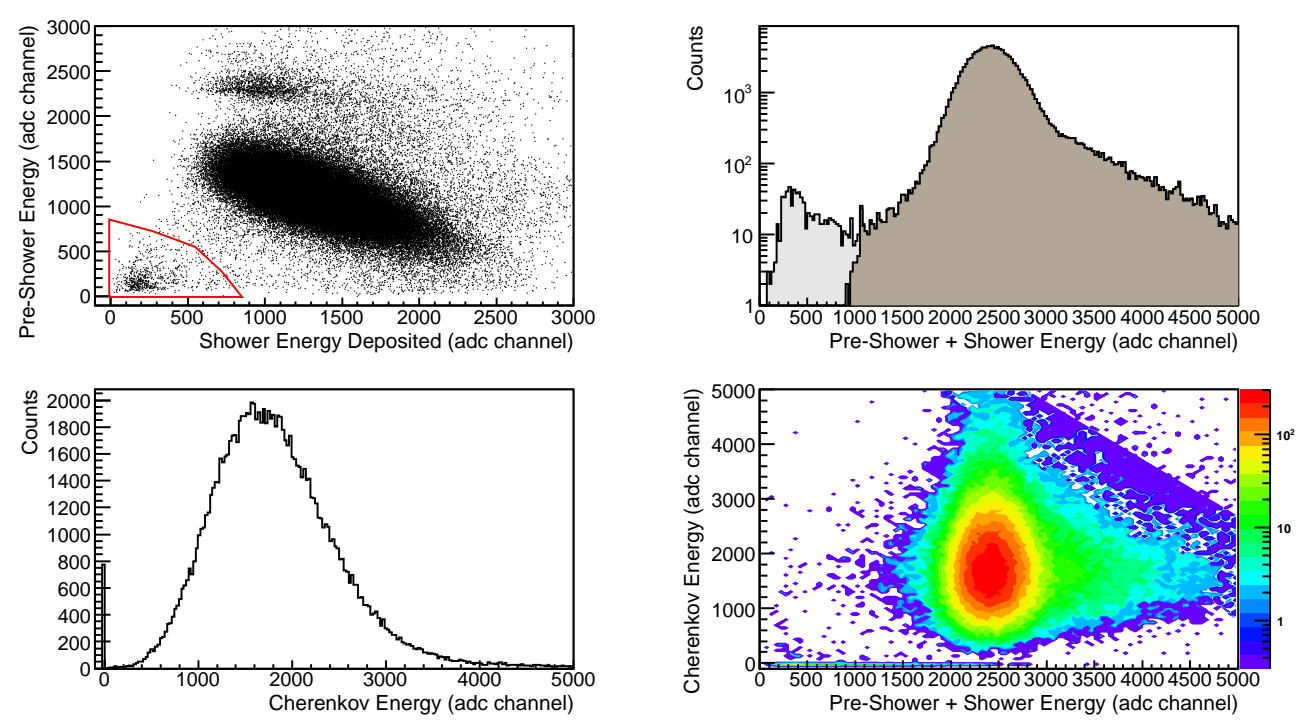

Figure 3.4: These histograms show the process of selecting electrons and rejecting pions from the tracks in the left HRS. Top-Left: The pre-shower energy deposited versus the shower energy deposited, with the electrons contained in the large distribution in the centre and the pions encircled by a $2 \mathrm{D}$ cut in the bottom left. The small upper distribution is due to a small calibration error of the preshower. Top-Right: Histogram showing the addition of the energy deposited in the pre-shower and shower counter. The events in the large peak are identified as electrons and the small peak (around channel 500) contains pions identified by the graphical cut. Bottom-Left: Plot showing the light output from the Cherenkov detector. Only the electrons deposit any significant amount of energy. Pions correspond to the large spike around zero. To remove these pions, only events where the Cherenkov ADC was above 50 channels were selected. Bottom-Right: Cherenkov energy versus the total shower counter energy.

\subsection{Right HRS Analysis}

Most of the Hall A Analyzer code used for the left HRS scattered electron analysis was also applied to the right HRS data. The same tracking and target coordinate reconstruction procedures were used, but the right HRS was optimised to detect protons, along with deuterons and pions by choosing the trigger appropriately. The comparative level of pions to protons was greatly reduced using the T5 events (see section 2.3.1), where a coincidence with the left HRS is required. Since no special PID detectors, such as a shower counter, were used in the right HRS, event identification was heavily reliant on Time of Flight (TOF) to the plastic scintillator trigger planes and energy deposited in the scintillator planes.

The speed $\beta=v / c$, measured using the TOF between the S1 and S2 scintil- 
lator planes and the coincidence time between the triggers of the left and right HRS are shown in 3.5. The particles in the right HRS were first identified and then the coincidence time was used to select (e,e'p) and (e,e'd) events and to estimate the random coincidence background.
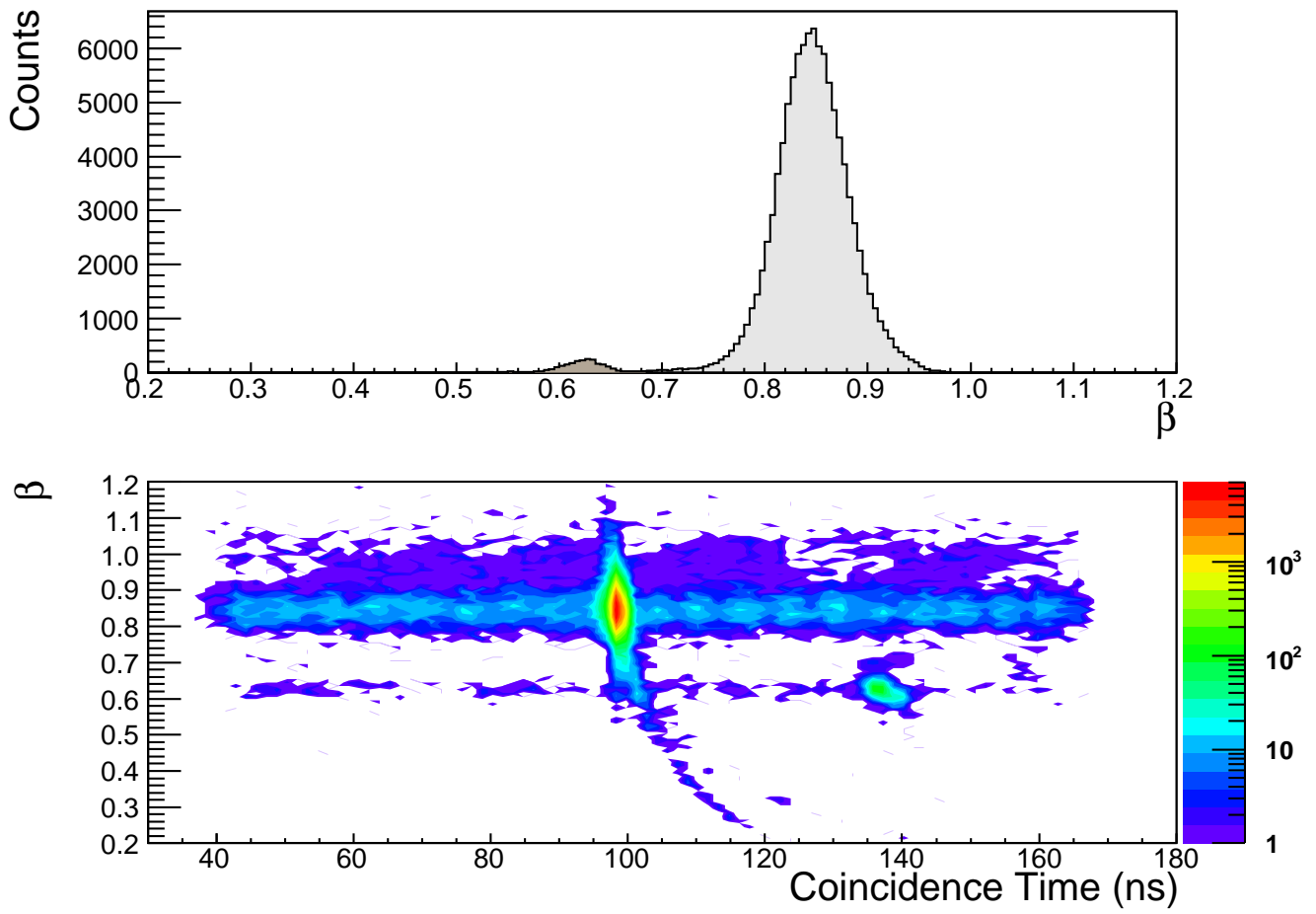

Figure 3.5: Top: The $\beta$ spectrum of the particles in the right HRS. The large peak to the right contains protons $(\beta \sim 0.85)$ and the smaller peak represent deuterons $\beta \sim 0.63$. Bottom: The coincidence time versus $\beta$. The coincidence time of the protons is around $100 \mathrm{~ns}$ and the deuterons are around $140 \mathrm{~ns}$.

Due to the restricted momentum range of the HRS, the particles are quite well separated when looking only at the $\beta$ spectrum, but the quality of the particle identification can be further improved if the energy deposited in the the S2 scintillator plane and the momentum of the track are used in conjunction with $\beta$ (see fig. 3.6). The $2 \mathrm{D}$ cut in the top plot rejects the majority of pions with a $\beta \sim 1$ that deposit a small amount of energy in the S2 scintillator plane. This correlated 2D data-set was not used to identify protons and deuterons because there is a wide distribution of energies deposited by both particles that extend beyond the full scale of the ADC. With the previous cut applied, the bottom plot, shows that the $\beta$ of the protons and deuterons is approximately constant over the small range of momentum acceptance. The two $2 \mathrm{D}$ cuts select the protons and deuterons corresponding to speeds of $\beta \sim 0.85$ and $\beta \sim 0.63$ respectively. 

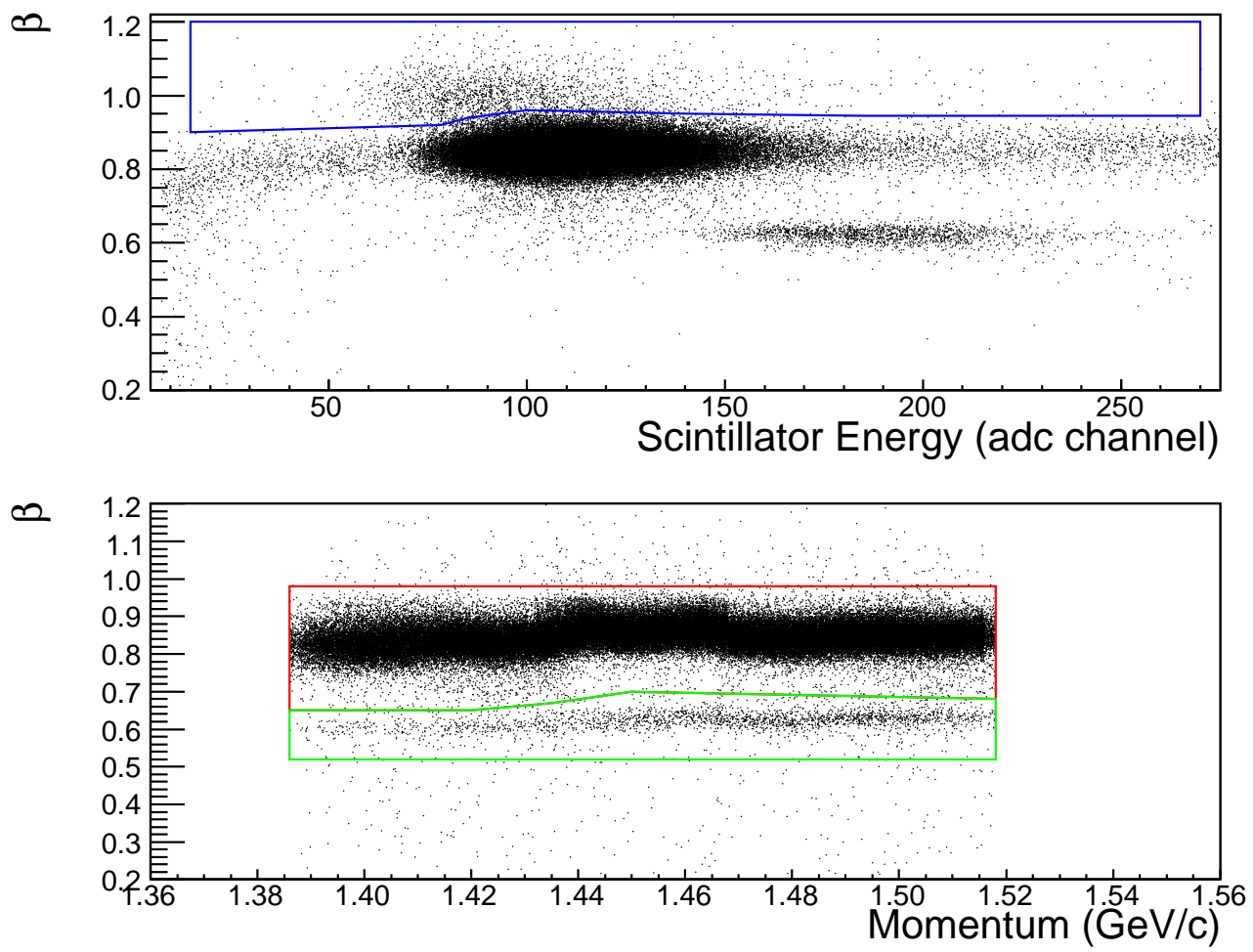

Figure 3.6: Top: $\beta$ versus deposited energy in the $\mathrm{S} 2$ scintillator plane. The majority of the pions are within the blue $2 \mathrm{D}$ cut. Bottom: $\beta$ versus the momentum of the track. Most of the pions have been removed from this plot and the red 2D cut selects the protons in the the main distribution and the green $2 \mathrm{D}$ cut selects the deuterons.

Applying the acceptance and particle identification cuts of both the HRSs to the coincidence time, the number of coincidence $\left(e^{\prime}, p\right)$ and $\left(e^{\prime}, d\right)$ events with background are obtained (see fig. 3.7). The $\left(e^{\prime}, p\right)$ (top plot) and $\left(e^{\prime}, d\right)$ (lower) coincidence events are contained within a peak of 100 ns and 140 ns respectively. Not all proton events could be fully eliminated by the $2 \mathrm{D}$ cuts and the remaining protons are cleanly seen in the small peak to the left in the $\left(e^{\prime}, d\right)$ coincidence time plot. When the coincidence time cuts (in red) are inserted around the correct peaks, the proton events are cleanly cut from the $\left(e^{\prime}, d\right)$ coincidence events. The background is only really apparent when a log scale is used on the number of events as in the $\left(e^{\prime}, p\right)$ coincidence time plot. The repeating up-down nature of this background is due to the radiofrequency (499 $\mathrm{MHz})$ time structure of the electron beam that arrives in Hall A. The intensity of background events is estimated by sampling on either side of the coincidence peaks. An integral was performed within the areas enclosed by the green lines and the average number of background events per histogram bin was calculated, thus allowing the number 
of background and real events within the cut zones to be estimated.
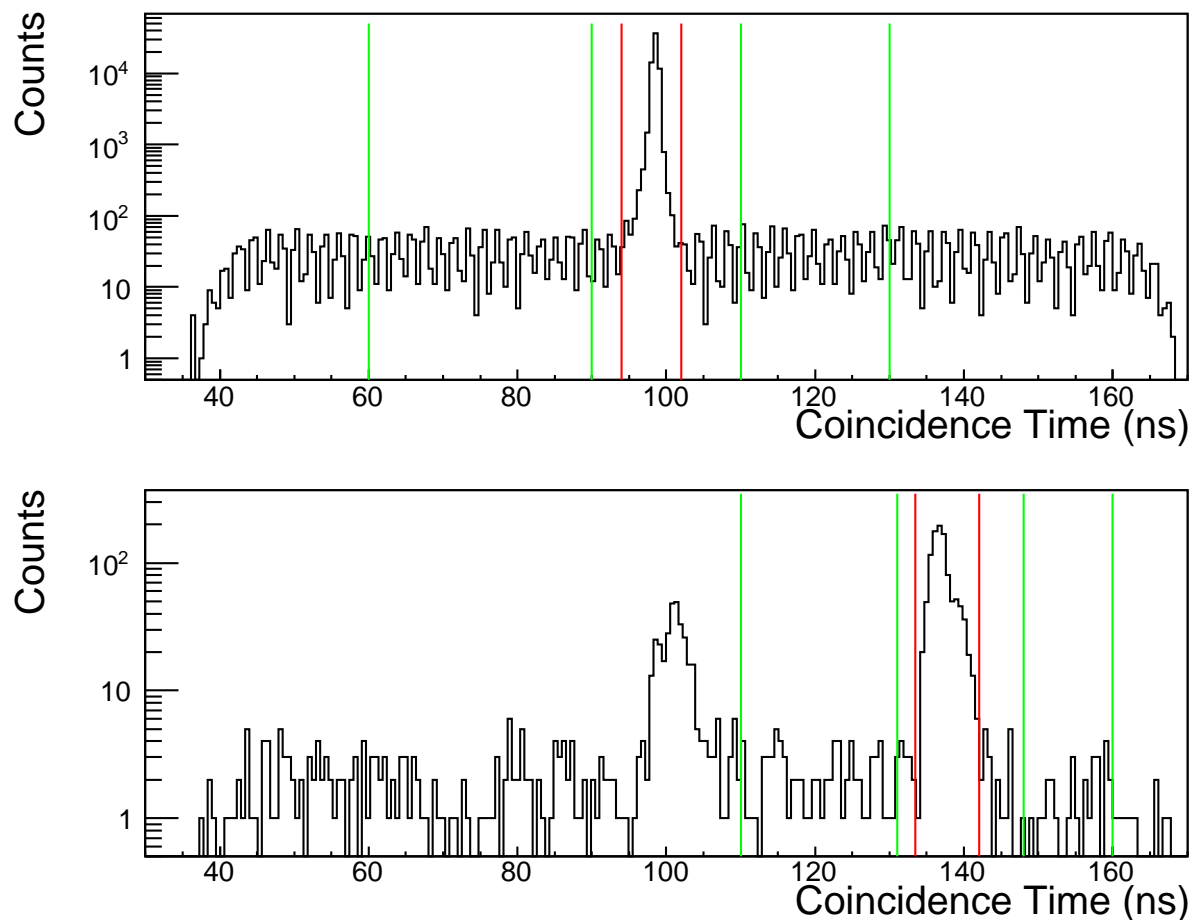

Figure 3.7: Top: The coincidence time (log scale) of the (e,e'p) events is selected within the red cut lines and the regions bordered by the green lines contain the events selected for background estimates. Bottom: The coincidence time plot for (e,e'd) events.

\subsection{1 ${ }^{12} C\left(e, e^{\prime} p\right)$ Kinematic Cuts}

The previous sections show the process of selecting the ${ }^{12} C\left(e, e^{\prime} p\right)$ and ${ }^{12} C\left(e, e^{\prime} d\right)$ reaction channels from the HRS data. The different kinematics chosen for this experiment were designed to favour NN SRC reactions over $\Delta$ excitation and meson exchange currents, but some of the ${ }^{12} C\left(e, e^{\prime} p\right)$ events are due to $\Delta$ excitation. Also, ${ }^{12} C\left(e, e^{\prime} p\right)$ reactions that leave the recoiling nucleus $\left({ }^{11} B\right)$ in the ground state do not leave enough missing energy $\left(E_{m}=\omega-T_{p}-T_{A-1}\right.$, where $\omega$ is the transferred energy, $T_{p}$ is the kinetic energy of the proton and $T_{A-1}$ is the kinetic energy of the recoiling nucleus) to account for a secondary proton or deuterium to emitted in a NN SRC reaction.

The influence of the ground state of ${ }^{11} B$ recoiling nucleus was removed by requiring the ${ }^{12} C\left(e, e^{\prime} p\right)$ missing energy to be above $32 \mathrm{MeV}$ (see fig. 3.8), removing $\sim 7000$ events. The wide distribution to the right of the missing energy spectrum contains events due to $\Delta^{+}$excitation and its subsequent decay to a proton 
and a $\pi^{0}$. To find suitable cuts to remove these events, the missing energy was plotted against the transferred energy $\omega$ and the angle of the recoiling nucleus. The energy transfer threshold to produce the $\Delta$ resonance at the experimental kinematics was around $0.95 \mathrm{GeV}$. Missing energy also varies with the recoil angle of the nucleus because the more the missing energy, the greater the momentum of the recoiling nucleus. Therefore the nucleus is more unlikely to be deflected backwards at higher missing energy. The $\Delta$ resonance could be seen most clearly when plotted against this recoil angle and cuts above $77^{\circ}, 84^{\circ}$ and $88^{\circ}$ were used to remove the $\Delta$ excitations for each kinematic settings of ${ }^{12} C\left(e, e^{\prime} p\right)$ missing momentum of 300,400 and $500 \mathrm{MeV} / \mathrm{c}$ respectively.
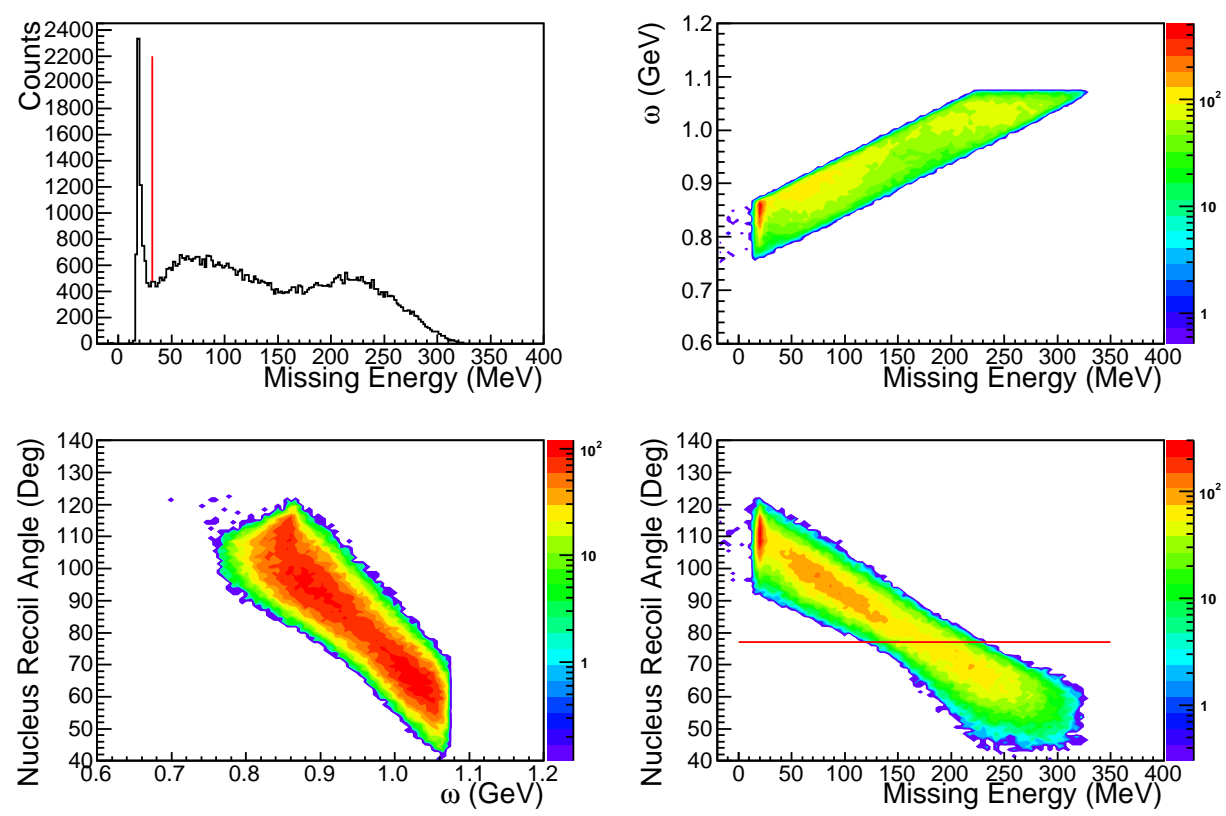

Figure 3.8: Top-Left: Missing energy of ${ }^{12} C\left(e, e^{\prime} p\right)$ reaction. The pronounced ground state peak at about $30 \mathrm{MeV}$ is removed by the cut (red line) at $32 \mathrm{MeV}$. In the continuum there are two broad peaks at $\sim 130$ and $\sim 240 \mathrm{MeV}$. TopRight: Transferred energy $\omega$ versus missing energy. Bottom-Left: Recoil nucleus angle versus $\omega$. Bottom-Right: Recoil nucleus angle versus missing energy. All histograms are from the first kinematic setting of ${ }^{12} C\left(e, e^{\prime} p\right)$ missing momentum of $300 \mathrm{MeV} / \mathrm{c}$. The pronounced ground state in missing energy spectrum peak at about $30 \mathrm{MeV}$ is removed by the cut (red line) at $32 \mathrm{MeV}$. In the missing energy continuum there are two broad peaks at $\sim 130$ and $\sim 240 \mathrm{MeV}$. The 130 $\mathrm{MeV}$ peak is at lower transferred energy and higher backward angles than the $240 \mathrm{MeV}$ peak, which is due to the $\Delta$ excitation. A cut above $77^{\circ}$ was used to cut the contributing $\Delta$ excitations. 


\subsection{BigBite Analysis}

The BigBite spectrometer was used to detect recoiling protons or deuterons in coincidence with the ${ }^{12} C\left(e, e^{\prime} p\right)$ or ${ }^{12} C\left(e, e^{\prime} d\right)$ events detected with the two HRSs. The other important objective was to the track the particles through the BigBite magnet and detector to determine the horizontal and verticle angles and the momentum of the particle at the reaction vertex.

\subsubsection{Bar Hit Position Calibration}

The hit position along the $\mathrm{dE}$ and $\mathrm{E}$ bars was calculated using the time difference obtained from the TDCs of the PMTs at each end of the scintillator bar. The following equation was used to determine the hit position $\left(y_{p o s}\right)$,

$$
Y_{\text {pos }}=C_{n} \times\left(\frac{\left(T_{L}-T_{R}\right)}{2}-T_{\text {offset }}\right)
$$

where $C_{n}$ is the effective speed of light and $T_{L}$ and $T_{R}$ are the left and right TDC signal and $T_{\text {offset }}$ is the time offset that is needed to centre the time difference spectrum around zero.

$\left(T_{L}-T_{R}\right) / 2$ for each $\mathrm{E}$ bar was analysed to find the leading and trailing edges ( $1 / 2$ full width half maximum of the height of the time difference spectrum when plotted with a y-axis $\log$ scale) as well as the centre position using ${ }^{12} C$ target production data to ensure that the whole length of the bar is illuminated due to the wide angular range of proton knockout (see fig. 3.9). The time offset corresponds to this centre position and once this is subtracted the spectrum is centred around zero. The effective speed of light is calculated by finding the factor needed to convert the time difference spectrum to a hit position spectrum with the physical width of the scintillator bar. 




Figure 3.9: Raw time difference spectrum for an E-plane Bar. The red lines are the leading and trailing edges of the raw time difference spectrum. The blue line is centre position of the raw time difference spectrum.

Since the time resolution of the $\mathrm{dE}$ plane bars is poorer than the $\mathrm{E}$ bars, due to being only $3 \mathrm{~mm}$ thick, the hit positions from the bars in the E plane were used to calibrate the hit positions of the $\mathrm{dE}$ bars, if the hits were within acceptable ranges of position and time of each other. The effective speed of light values and the time offsets of the $\mathrm{dE}$ bars were obtained from linear fits of the $\mathrm{dE}$ bar time difference plotted against the hit position of the corresponding $\mathrm{E}$ bar (See fig. $3.10)$. 


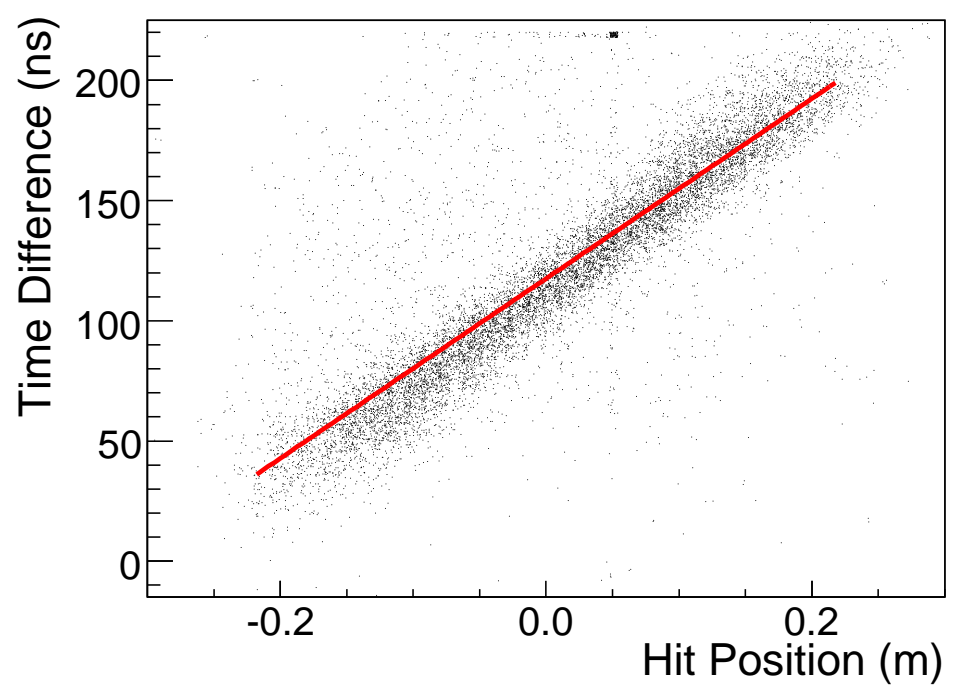

Figure 3.10: Time difference of a $\mathrm{dE}$ bar plotted against the hit position of corresponding $\mathrm{E}$ bar that is located after the $\mathrm{dE}$ bar. From the parameters of the linear fit, the $\mathrm{dE}$ time difference is calibrated to produce the hit position along the bar.

\subsubsection{ADC Calibration}

The calibration of the scintillator planes ADCs requires three main steps. The first and simplest step is to find the ADC pedestals for all auxiliary and trigger PMTs. These were determined by finding the channel number of each ADC for events with no corresponding TDC hit and new variables for the left and right ADCs with their pedestal subtracted were formed. The next step was to correct to the attenuation lengths of each scintillator bar and take the average of the left and right ADCs. The final step was to convert the ADC output of channel number into $\mathrm{MeV}$, allowing the summation of the energy deposited in different scintillator bars into the total energy deposited for each scintillator plane.

\section{Attenuation Lengths}

There is a characteristic drop in the measured energy deposited along the length of each scintillation bar, due to decrease in intensity of scintillation photons with distance. This effect was accounted for after measuring the attenuation length of each bar.

The hit position along the bar can also be estimated from the the left and right ADCs using the equation $Y_{A D C}=\frac{L}{2} \exp \left(\frac{A_{R}}{A_{L}}\right)+Y_{A D C O f f}$, where $Y_{A D C}$ is the hit position along the bar, $L$ is the attenuation length, $A$ is either the left 
or right ADC channel and $Y_{A D C O f f}$ is the position offset due to the difference in the ADC gains. The attenuation lengths and ADC offsets were extracted by fitting linear fits to plots of the hit positions along the bar derived from the time difference versus the raw hit positions from the ADCs (see fig. 3.11).

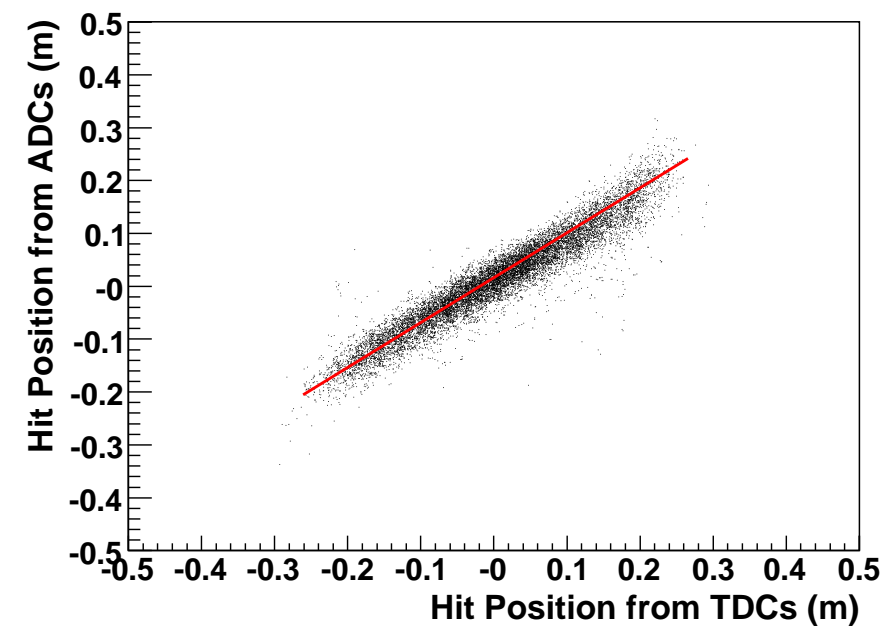

Figure 3.11: Linear fit to particle hit position along bar from time difference against the raw hit position derived from the ADCs. The gradient from the straight line fit gives the attenuation length of the scintillator bar and the intercept is equal to the natural log of the ratio of the left and right ADC conversion.

The geometric mean of the left and right ADCs used the values of the attenuation lengths of the scintillator bars using the equation:

$$
A_{\text {Mean }}=\sqrt{A_{L} \exp \left(-d_{L} / L\right) \times A_{R} \exp \left(-d_{R} / L\right)}
$$

where $d_{L, R}$ is the distance to the left or right PMT. This expression is more closely related to the energy deposited in the bar than the arithmetic average, which takes no account of the attenuation length of the scintillator bars (see fig. 3.12). These dE against E bar energy deposited plots show that correction for attenuation length produces sharper energy distribution relationships between the $\mathrm{dE}$ and $\mathrm{E}$ bar for the protons in particular and the other particles too. 

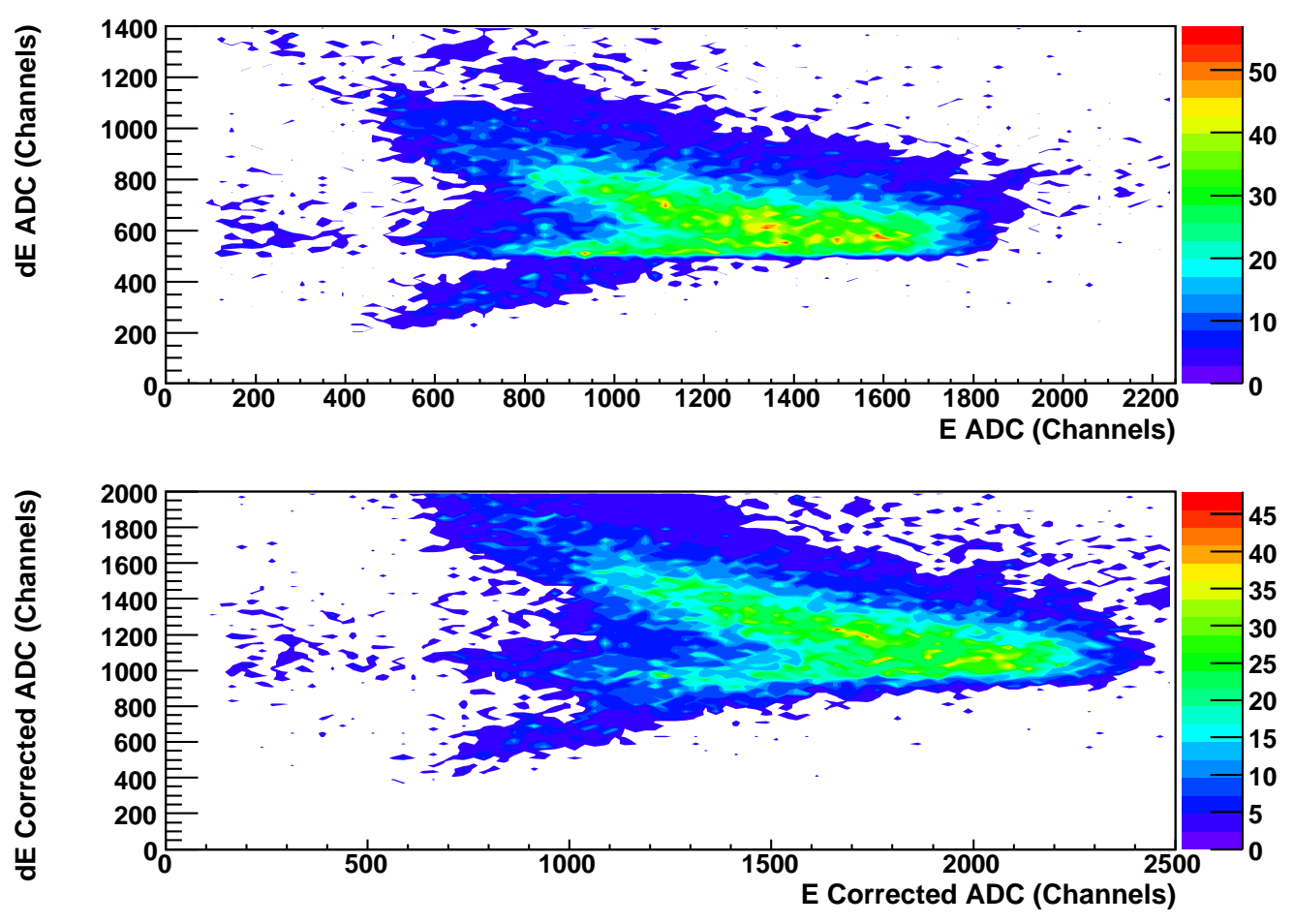

Figure 3.12: Top: arithmetic mean of the left and right ADCs of a dE scintillator bar versus the arithmetic mean of the left and right ADCs of a E scintillator bar. Bottom: Same as top, but the arithmetic mean has been replaced with the geometric mean of the left and right ADCs with correction for the attenuation length of the scintillator bars.

\section{ADC Gains}

Different particles can be identified by looking at the energy deposited in the $\mathrm{dE}$ layer versus the energy deposited in the $\mathrm{E}$ layer. This is due to the different energy loss of charged particles of different mass travelling through the scintillator medium at the same momentum. Figure 3.12, which shows the dE vs E energy deposition can be explained in terms of the momentum of the particle (proton). At low momentum the proton deposits a large amount of energy due to electronic and nuclear scattering and therefore it slows down quickly, travelling only a short distance. Therefore, the energy deposited by low momentum protons is relatively high in the dE layer, but low for the E layer due to the small distance travelled. As proton momentum increases, the rate of energy loss decreases causing the energy deposited to decrease, but the energy deposited in the E layer increases due to increase of distance travelled by the proton until it breaks through both layers. As proton momentum increase above this breakthrough momentum (about 350 
$\mathrm{MeV} / \mathrm{c}$ ), the energy deposited in both scintillator layers decreases.

Therefore, to identify particles using the energy deposited in the trigger plane, all the combinations of $\mathrm{dE}$ and $\mathrm{E}$ bars are calibrated by aligning the breakthrough points so that a plot of $\mathrm{dE}$ vs E energy deposited can be formed using all bars. This was done primitively during the run by adjusting the voltages going to each of the PMTs to set the breakthrough point to channel number 1500 for the $\mathrm{dE}$ bars and channel number 2500 for the E bars. This was further improved, after attenuation energy losses were taken into account, by lining up each breakthrough point carefully for each $\mathrm{dE}$ bar versus the $\mathrm{E}$ bar behind it by adjusting a scale factor to convert the ADC channel number into $\mathrm{MeV}$ to make the breakthrough point equal to $3.3 \mathrm{MeV}$ and $60 \mathrm{MeV}$ in the $\mathrm{dE}$ and $\mathrm{E}$ bars respectively which are appropriate to the thicknesses of these bars, based on Monte-Carlo simulations of BigBite (see section 3.4.5).

\subsubsection{TOF Calibration}

The time-of-flight (TOF) variable for the $\mathrm{dE}$ and E scintillator bars was formed using the average of the left and right TDCs. The previously measured time difference offset was subtracted from this variable to account for the time difference between the left and right TDCs. The TOF was converted from TDC channels into seconds using the conversion gain of $35 \times 10^{-12}$ (s/TDC channel number). The start time of the event, the time for the electron to scatter from the reaction vertex and travel the path length to the $\mathrm{S} 2$ scintillator plane and trigger the electronics for the left HRS, was subtracted from the BigBite time spectra.

The next calibration step was to align the TOF of the $\mathrm{E}$ and $\mathrm{dE}$ scintillator bars. This was done by comparing the TOF for each pair of adjacent $\mathrm{dE}$ and E bar pairs, which should be the very similar considering that they are placed immediately next to each other. The $\mathrm{dE}$ bars overlap the E bars equally allowing a cross-check of alignment (see fig. 3.13). The TOF difference between the dE and $\mathrm{E}$ bars was linearly correlated with the total TOF of either the $\mathrm{dE}$ and $\mathrm{E}$ bars. The reason for this is unclear, but it might be to do with an error in the way the TDC electronics was set-up. This effect was quantified by a linear fit to the $\mathrm{dE}$ and $\mathrm{E}$ bar TOF difference plotted against the $\mathrm{dE}$ bar TOF and corrected for by adding a small scale factor into the dE bar TOF as well as the total time offsets for all the scintillator bars derived from all the intercepts of the straight line fits. 

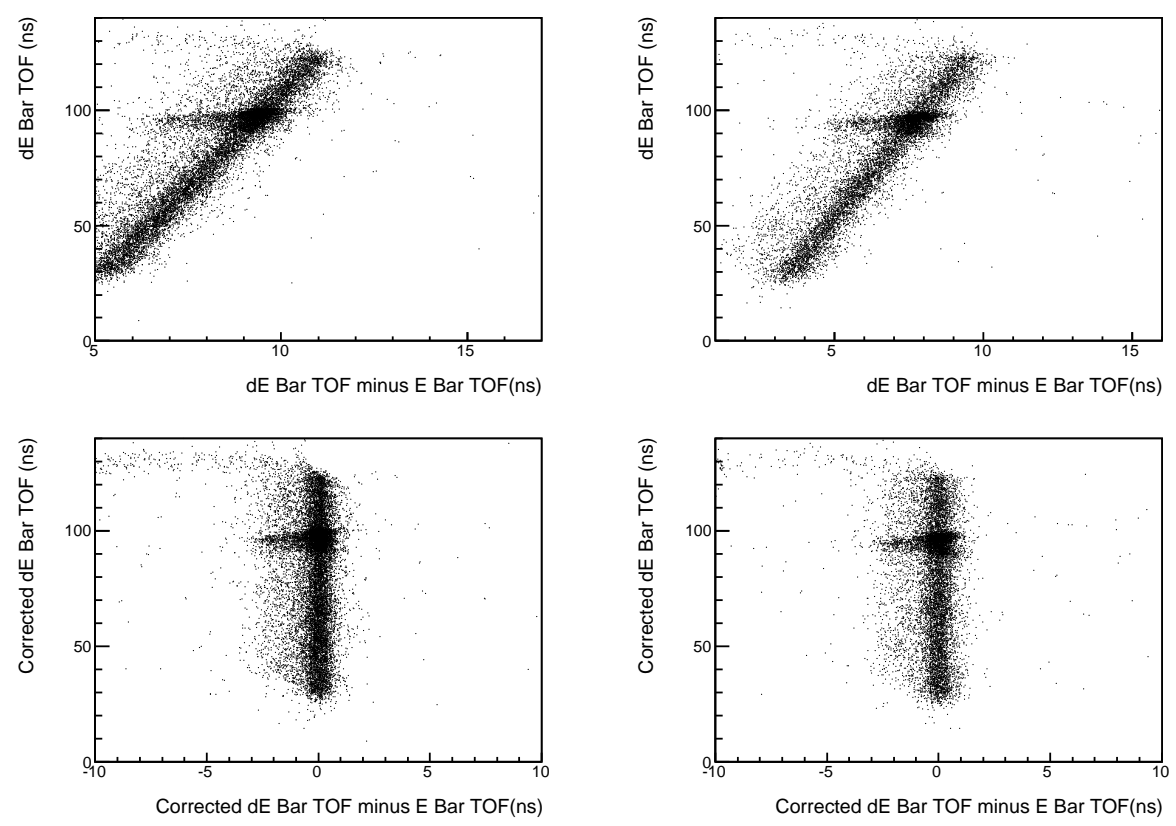

Figure 3.13: Top-Left: dE bar TOF vs dE and E bar TOF difference for same bar number pairings. Top-Right: dE bar TOF vs E bar TOF difference for oddeven bar number pairings. The bottom histograms are the same, except the TOF offsets and $\mathrm{dE}$ scale factors have been implemented to make the TOF differences between $\mathrm{dE}$ and $\mathrm{E}$ bars and the bars on the same plane all centred around zero.

Finally the TOF offset for the E plane was calculated by finding the difference between the measured TOF and the calculated TOF between the target and the bar hit using the momentum measured from the particle bend in the magnetic field of BigBite.

\subsubsection{Tracking and Momentum Calculation}

The aim of the simple tracking procedure used in the BigBite data analysis was to reconstruct the initial track of the detected particle at the target. The momentum vector for each track was obtained after determining the 'in' and 'out' of plane angles and the magnitude of the momentum. Lorentz vectors representing protons and deuterons were formed for all tracks with the idea of using particle identification cuts to select the relevant variable during later analysis stages. The momentum and angular resolution of the track reconstruction in BigBite is poor, especially compared to that of the High Resolution Spectrometers. The detector package of BigBite consists of two planes of segmented scintillators separated by $1 \mathrm{~m}$ and the position resolution in the trigger plane is approximately $5 \mathrm{~cm}$ in the vertical and horizontal directions. Given the relatively poor resolution of BigBite, 
it provided no meaningful information on the reaction vertex, which was determined by the HRSs. Multiple tracks were not thrown away at any stage in the BigBite analysis, but for every triple coincidence event only one track remained after the acceptance and raw ADC and TDC detector cuts.

The in-plane angle $(\theta)$ of the particle trajectory at the target is equal to the in-plane angle of the particle passing through the auxiliary and trigger planes. The first step to obtain $\theta$, was to calculate the position vector of the particle hit in the E plane in the hall frame by projecting the scintillator bar hit position from the centre of the bar along its angle (see fig. 3.14). In the hall frame, the $\mathrm{z}$ axis is along the beam direction towards the beam dump and the $\mathrm{y}$ axis is the vertically upwards towards the hall ceiling and the $\mathrm{x}$ axis follows from the relation $\hat{z}=\hat{x} \times \hat{y}$ with the origin located at the target. $\theta$ is therefore calculated using $\mathrm{x}$ and $\mathrm{z}$ components of the position vector of the $\mathrm{E}$ bar hit in the hall frame using the equation $\theta=\tan ^{-1} \frac{x_{\text {Hall }}}{z_{\text {Hall }}}$.

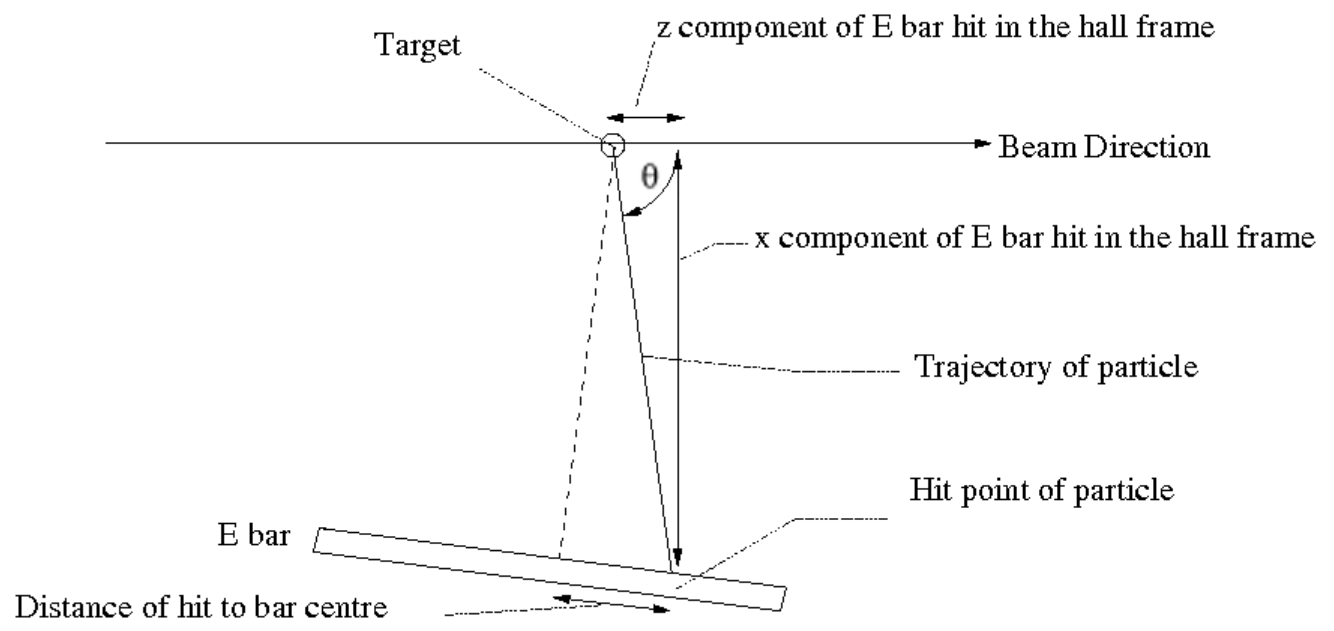

Figure 3.14: Diagram of a particle hit in a $\mathrm{E}$ bar and the terms necessary to calculate the in-plane angle $(\theta)$ in the hall frame.

An approximate method of calculating the momentum and out-of-plane angle $(\phi)$ at the target was used that worked by tracking the particle trajectory from the auxiliary and trigger planes through the magnetic field and back towards the target to find the radius of curvature of the bend in trajectory of the charged particle due to the magnetic field. The particle path was assumed to have passed through the horizontal centre of the magnet to simplify the problem to two dimensions, the dispersive axis (x axis) and the particle direction ( $\mathrm{z}$ axis) (see fig. 3.15). First the particle trajectory through the scintillator detector planes was calculated using the coordinates of the auxiliary and trigger plane bars hits. The 
position resolution of the particle path in the dispersive direction was improved in the trigger plane due to the $\mathrm{dE}$ bars overlapping two $\mathrm{E}$ bars. The vector of the particle path through the scintillator planes was then used to find the intersects on the exit magnetic field boundary plane (angled at $25^{\circ}$ ) and the centre-line plane in the magnetic field, which are described by two axis vectors and a position vector of the plane in Hall A.

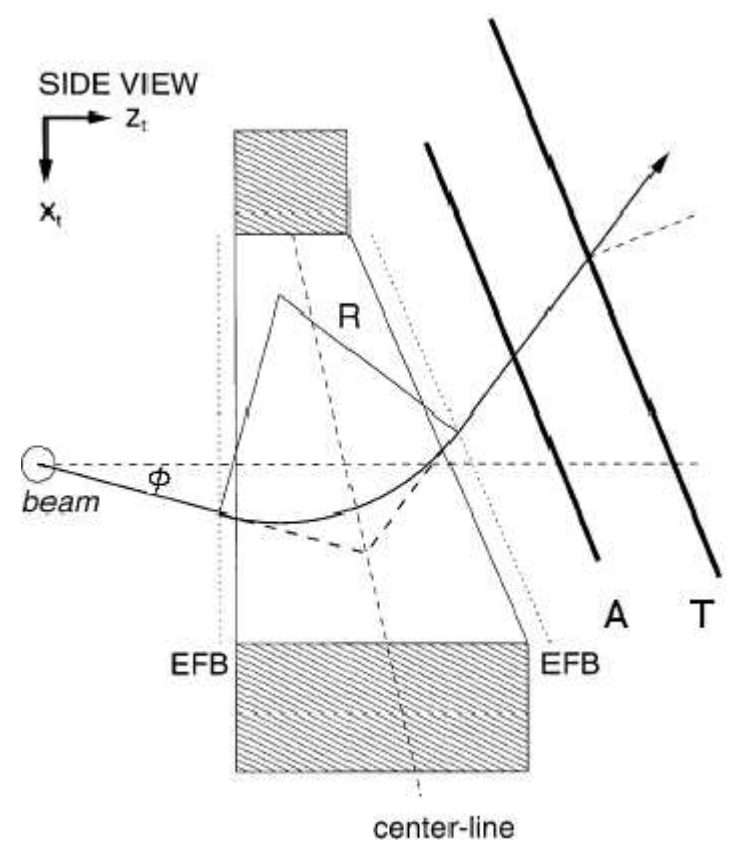

Figure 3.15: Side view of a particle trajectory through the BigBite magnetic field and auxiliary and trigger planes. The momentum and $\phi$ of the track was calculated by tracking the particle path through the scintillator planes back through the magnetic field and to the target.

The initial particle trajectory is the vector between the origin and centreline intersect and the out-of-plane angle $\phi$ is contained within this vector. The next step was to calculate the intersect of this initial particle vector and the front magnetic field boundary using the same process as before. The centre of the circle corresponding to the arc that describes the curvature of particle path through the magnetic field was obtained by intersecting the normals of the initial and detector particle trajectory vectors at the points where the particle enters and exits the magnetic field boundaries. The radius of curvature of particle bend in the magnetic field is the distance between the centre of the circle and one of the magnetic field intersect. The momentum (in $\mathrm{GeV} / \mathrm{c}$ ) was calculated using this radius of curvature $\left(R_{c}\right)$ and the strength of the magnetic field $(|B|)$ using the non-relativistic equation $p=0.3 R_{c} B$, which is derived from balancing the 

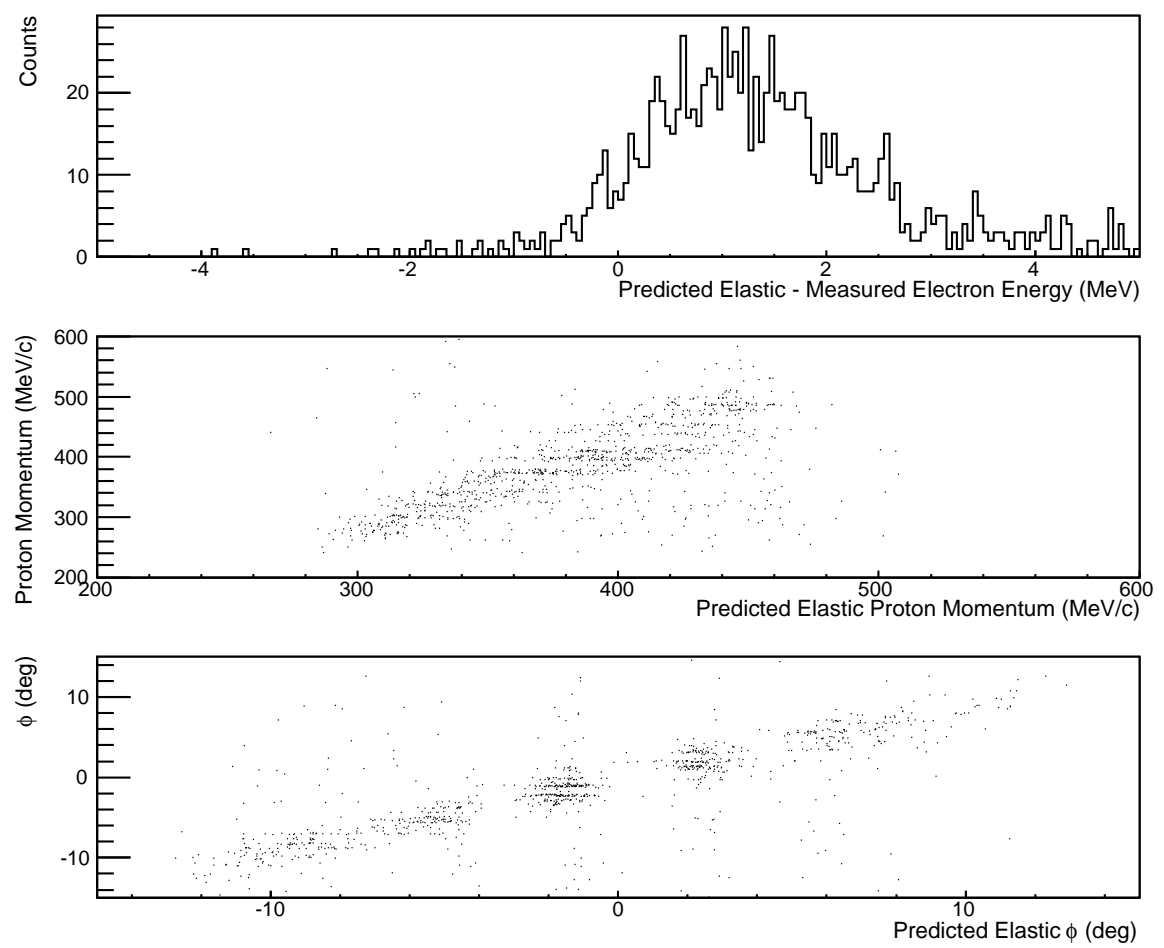

Figure 3.16: Top: The deviation between the scattered electron energy measured in the Left HRS and the electron energy predicted using for an elastic reaction using the initial electron energy and scattering angle. Middle: The measured elastic proton momentum versus the measured proton momentum in BigBite. Bottom: The measured elastic proton out-of-plane $\phi$ versus the measured $\phi$ in BigBite.

centripetal force $F_{c}=m v^{2} / R_{c}$ with the Lorentz force $\vec{F}=\vec{B} e \vec{v}$, where $e$ is the magnitude of the electron charge and is responsible for the 0.3 constant using the $\hbar=c=1$ particle kinematics convention. The momentum was calibrated by finding the location of the front and exit magnetic field boundaries using elastic hydrogen data ${ }^{2} H\left(e, e^{\prime} p\right)$. To ensure only elastic events were selected, necessary for obtaining the momentum and trajectory of the proton through BigBite, a cut was applied to the deviation of the measured scattered electron energy to the predicted elastic electron energy, given by $E_{f}=E_{i} /\left(1+\frac{2 E_{i}}{M_{p}} \sin ^{2} \frac{\theta}{2}\right)$ (see top of fig. 3.16). The calibration of the out of plane angle $\phi$ and the momentum response of BigBite was performed by varying the location of the centre line and the width of the magnetic field boundaries respectively (see fig. 3.16) until they were within good agreement of the measured elastic data.

For each track there is a combination of three scintillator bars hit, one in each scintillator plane. For each combination of auxiliary, $\mathrm{dE}$ and $\mathrm{E}$ bars there is 
only one unique momentum and $\phi$ value using this tracking method. The coarse granularity of the tracking detector leads to the artificial pronounced measured quantisation of the momentum and $\phi$ (see fig. 3.17). There are only a certain amount of possible scintillator bar combinations, limited by the momentum and the out-of-plane acceptances. To eliminate tracks caused by accidental hit occurring in one or two of the bars, the distribution of real tracks was selected from histograms of the auxiliary bars hit against the $\mathrm{dE}$ and $\mathrm{E}$ bar combination (see $3.17)$.
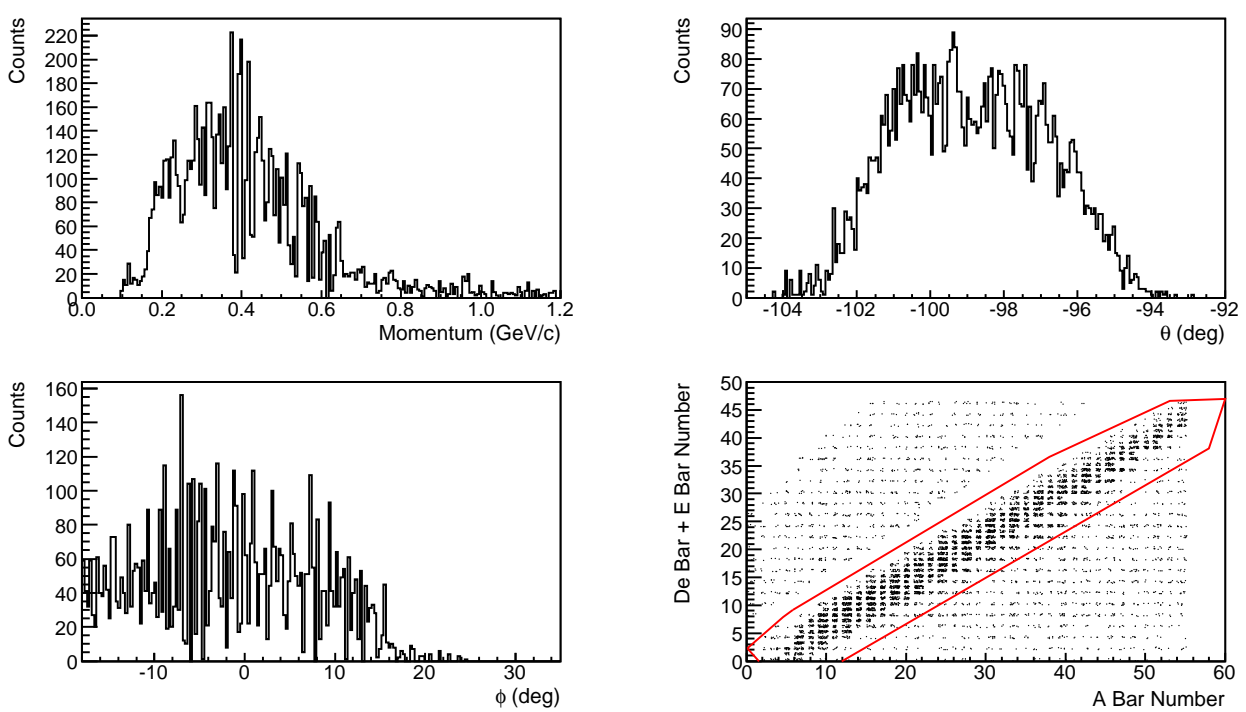

Figure 3.17: Top-Left: Momentum distribution in BigBite. Top-Right: In-plane angle $\theta$. Bottom-Left: Out-of-plane angle $\phi$. Bottom-Right: Auxiliary bar number plotted against the sum of $\mathrm{dE}$ and $\mathrm{E}$ bar number. The real tracks in the main linear distribution are encircled by the red graphical cut and the other bar combinations outside this distribution are due to uncorrelated hits.

\subsubsection{Geant4 Simulation}

The Geant4 simulation of BigBite by John Annand [7] was updated to include deuterons and the results are useful for the understanding of the BigBite event selection and yield extraction. The energy deposited in the $\mathrm{dE}$ and $\mathrm{E}$ bars for protons and deuterons is shown in fig. 3.18. The breakthrough points for both particles were used to calibrate the ADC channel number to energy deposited in $\mathrm{MeV}$ for $\mathrm{dE}$ and $\mathrm{E}$ bar hits. 


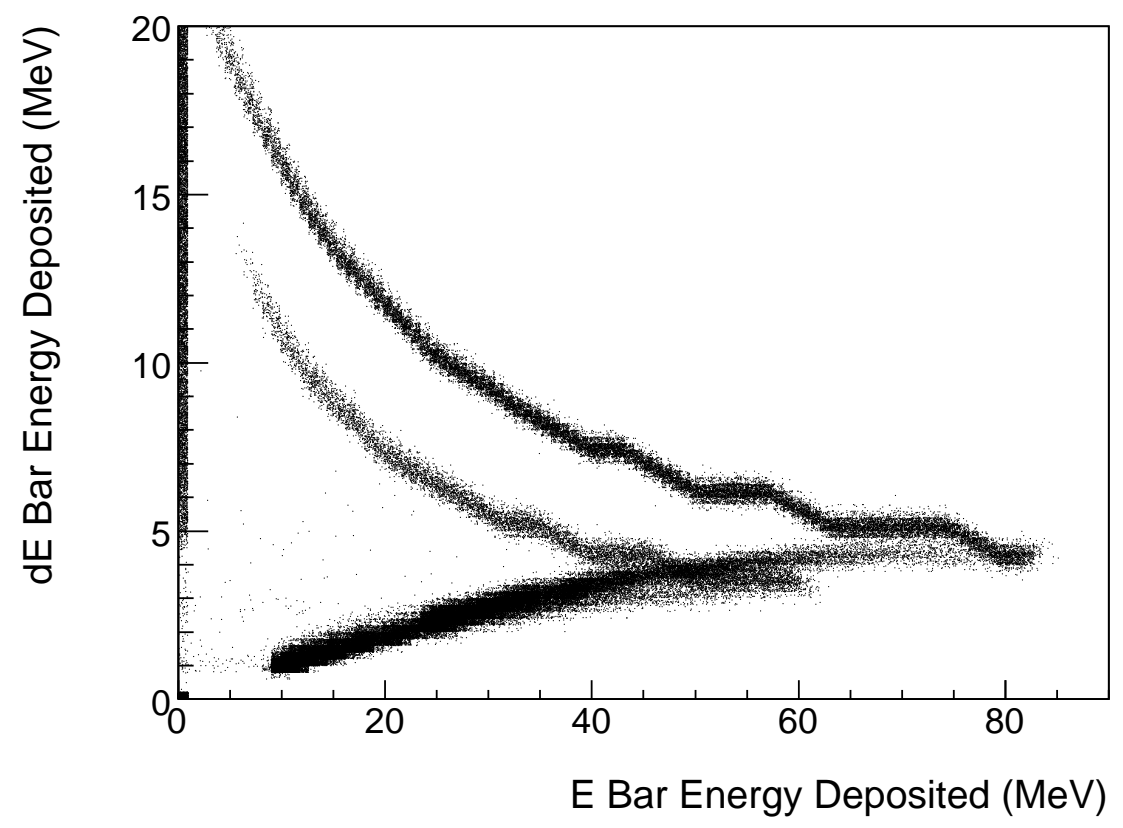

Figure 3.18: The dE bar energy deposited versus E bar energy deposited for protons (smaller spectrum with a breakthrough point of $\mathrm{dE}$ and $\mathrm{E}$ bar energy deposited of $3.5 \mathrm{MeV}$ and $60 \mathrm{MeV}$ respectively) and deuterons (larger spectrum with a breakthrough point of $\mathrm{dE}$ and $\mathrm{E}$ bar energy deposited of $4.5 \mathrm{MeV}$ and 83 $\mathrm{MeV}$ respectively). The wiggle in the $\mathrm{dE}$ vs E spectrums for both particles is due to the propagation step size of the particle's path, which is a little too long considering the small thickness of the $3 \mathrm{~mm}$ dE bar.

The momentum and tracking method described in section 3.4.4 was used in the BigBite simulation, enabling the comparison of this approximate momentum to the generated momentum. The generated and approximated momenta for protons and deuterons are displayed against the total energy deposited in the $\mathrm{dE}$ and $\mathrm{E}$ bars in 3.19. The generated momentum versus energy deposited in the trigger plane (top left) was used find the momentum $(\sim 360 \mathrm{MeV})$ that protons should breakthrough both layers of scintillator. The upper right plot shows the approximate momentum versus energy deposited using the actual hit coordinates in the scintillator bars, while the lower left uses the approximated momentum calculated with realistic detector hit coordinate resolution. These two plots show that the momentum calculated using this method does give a good approximation, but as expected, the detector resolution has a significant effect on the momentum resolution. The lower right plot shows the experimental energy deposited versus momentum, calibrated to match the momentum breakthrough point of the proton. The shape and resolution of the proton spectrum agrees closely with the predicted proton spectrum using the same approximation method 
and detector coordinates in the lower left plot. There are too few experimental deuterons to do a proper comparison with simulation, but there is a definite distribution of deuterons where they are predicted to be.
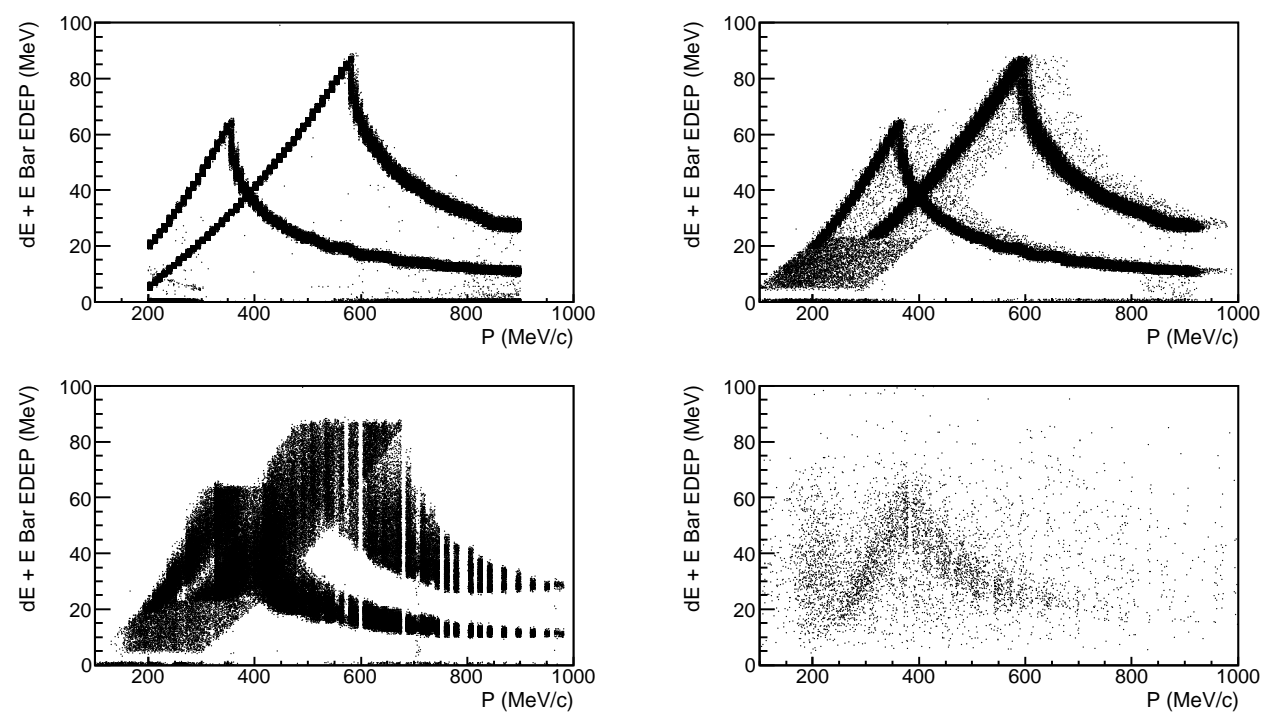

Figure 3.19: BigBite Geant4 simulation of dE plus E bar energy deposited versus generated momentum (top-left), approximated momentum using actual particle hit coordinates in the scintillator bars (top-right), approximated momentum using the simulated detector scintillator hit coordinates (bottom-left). Bottom-right: Experimental dE plus E bar energy deposited versus momentum.

The deviations in generated and simulated detector measurement of the momenta of protons and deuterons versus the generated momentum are shown in fig. 3.20. The width of the momentum deviation increases with the magnitude of the momentum, which is due to the approximate nature of the momentum calculation method. As the momentum of a particle in BigBite increases, the radius of the particle curvature in the magnetic field also increases, meaning the resolution of the calculated momentum increases due to the decrease in accuracy in finding the centre of curvature. The momentum deviation versus generated momentum is banded into slanted thin distributions. This is because the generated momentum on the $\mathrm{x}$-axis has a one-to-one linear relationship with the same generated momentum in the $y$-axis that is offset over small ranges of generated momentum by the particular simulated detected momentum that is applicable for that range of momentum.

The deviation in the generated TOF and the TOF calculated from the simulated detector momentum versus momentum for protons and deuterons is also shown in fig. 3.20. For protons and deuterons there is a TOF deviation offset 
of $\sim 2 n s$, which is partly due to a small underestimation of the the distance $\sim 20 \mathrm{~mm}$ over the average path length of $\sim 3 \mathrm{~m}$ of the approximate momentum method, but the most significant part is due energy losses in the target and auxiliary plane. The width of the TOF deviation is greater for deuterons than it is for protons due to the slower velocities and higher TOF at similar momentum. The TOF deviation also narrows in range as momentum increases for both particles, which is contrary to the generated and simulated momentum deviation. This is because of the total TOF for both particles decreases as momentum increases. The TOF deviations are also banded due to the same reasons as the momentum deviations. Over small ranges of momentum, this effect can shift the peak of the TOF deviation, if it catches the bottom edge of one of the TOF deviation bands compared to an upper edge of the neighbouring band.

To check what effects this had on the experiment, the momentum of the protons and deuterons were restricted to the missing momentum range of the ${ }^{12} \mathrm{C}\left(e, e^{\prime} p\right)$ reaction for the three kinematic settings, which are $150-450 \mathrm{MeV} / \mathrm{c}$, $250-550 \mathrm{MeV} / \mathrm{c}$ and $350-650 \mathrm{MeV} / \mathrm{c}$ for CK1, CK2 and CK3 respectively (see fig. 3.21). Over the this momentum range of $300 \mathrm{MeV} / \mathrm{c}$, the position of the peaks are all around $\sim 2.5 \mathrm{~ns}$ and the widths for the protons and deuterons are fairly stable with values across the different kinematic settings of $\sim 1.75-2 \mathrm{~ns}$ and $\sim 4 n s$ respectively. Therefore the widths for protons and deuterons should be broadly similar across the different kinematic settings but due to the low statistics of the experiment combined with the banding of TOF minus calculated TOF, the widths and positions of the protons and deuterons should also have some unpredictability and variance.

\subsubsection{BigBite Event Selection}

${ }^{12} C\left(e, e^{\prime} p p\right)$ and ${ }^{12} C\left(e, e^{\prime} p d\right)$ events require clear particle identification of protons and deuterons in BigBite in coincidence with ${ }^{12} C\left(e, e^{\prime} p\right)$ events detected using the left and right HRS. The PID for BigBite relied on the TOF of the particles between the target and the E plane and the energy deposited in the $\mathrm{dE}$ and E layers of the trigger plane, which are characteristic of the particle and its momentum.

\section{Initial Cuts}

Events with no BigBite tracks were rejected and the ADCs of the $\mathrm{dE}$ and E scintillator bars were required to be greater than the ADC pedestal channel number 



Figure 3.20: Top left: The deviation in simulated detected momentum from the generated momentum against the generated momentum for protons. Top right: The same as top left, but for deuterons. Bottom left: The deviation in simulated detected TOF from the generated TOF against the generated momentum for protons. Bottom right: The same as bottom left, but for deuterons.
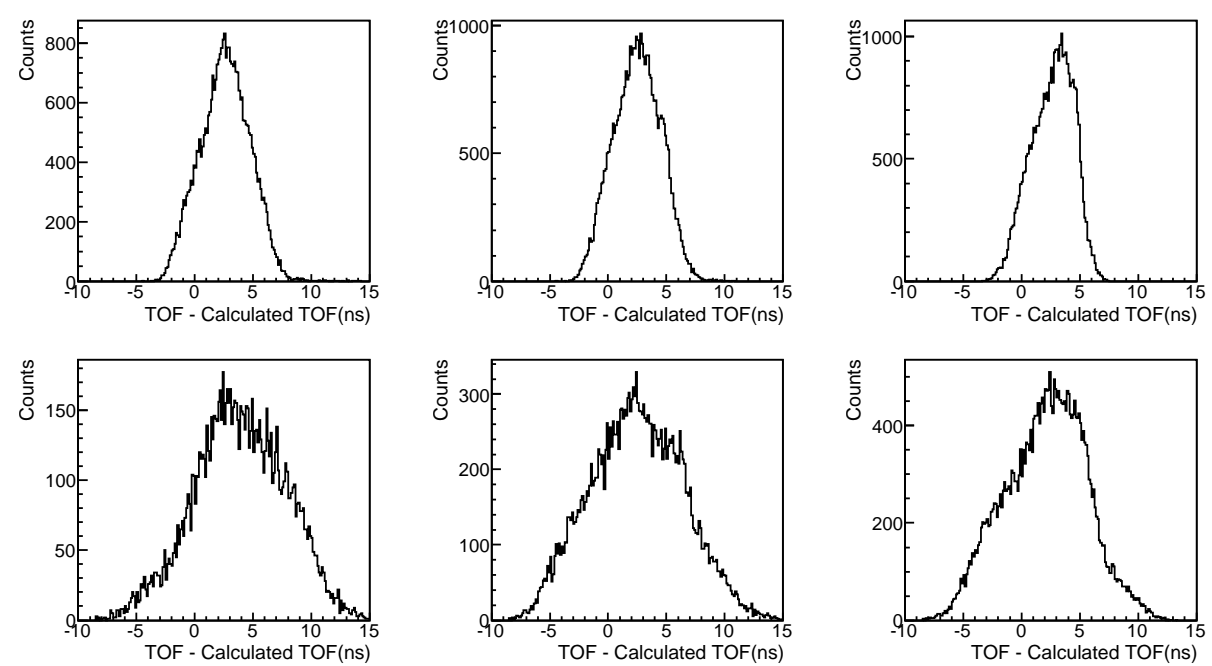

Figure 3.21: Top: The deviation in simulated detected TOF from the generated TOF for protons with generated momentum cuts applied on the three histograms left to right of $150-450 \mathrm{MeV} / \mathrm{c}, 250-550 \mathrm{MeV} / \mathrm{c}$ and $350-650 \mathrm{MeV} / \mathrm{c}$ to reproduce the three kinematic settings of CK1, CK2 and CK3 respectively.Bottom: Same as the (top), but deuterons were used instead of protons in the simulation. 
to ensure that only real bar hits were kept. The TDCs also had a cut of above a hundred channels to ensure that both TDCs in a each bar had registered a signal. The bar hit position and the in-plane angle theta were required to be within the length of the bar and acceptance of the BigBite magnet. Only a limited number of auxiliary, dE and E bar combinations formed real tracks (see section 3.4.4 and fig. 3.17). Events with BigBite tracks with momentum lower than $200 \mathrm{MeV} / \mathrm{c}$ were also rejected. This was because protons require a momentum of at least 200 $\mathrm{MeV} / \mathrm{c}$ (see fig. 3.23), to have enough energy to pass through the $\mathrm{dE}$ scintillator bars and deposit energy in the E plane scintillator bars. A cut on the auxiliary to trigger plane TOF peak, between 7-38 ns, was used to discard background.

\section{Calculation of Energy Deposited in the Trigger plane}

Separating protons and deuterons is very difficult using only the energy deposited in the $\mathrm{dE}$ and $\mathrm{E}$ scintillators of the trigger plane. This is because the $\mathrm{dE}$ vs $\mathrm{E}$ distributions of the protons and deuterons are similar at lower momenta and actually overlap when the momentum of either exceed the breakthrough point of the $\mathrm{dE}$ and E scintillators. The position of the particle hit along the $\mathrm{dE}$ vs E distribution corresponds to a particular momentum for a proton or deuteron. The momentum of the particle, determined by measuring the bend of the particle track through the magnet, was used to calculate the hypothetical energy deposited through ionisation in the $\mathrm{dE}$ and $\mathrm{E}$ scintillators for a proton, deuteron or a pion using the Bethe-Bloch equation. These calculated and measured $\mathrm{dE}$ and $\mathrm{E}$ bar energies were used to separate the protons and deuterons.

The energy loss of a charged particle of a particular medium can be calculated using the Bethe-Bloch formula

$$
-\frac{d E}{d x}=K z^{2} \frac{Z}{A} \frac{1}{\beta^{2}}\left[\frac{1}{2} \ln \frac{2 m_{e} c^{2} \beta^{2} \gamma^{2} T_{\max }}{I^{2}}-\beta^{2}-\frac{\delta(\beta \gamma)}{2}\right]
$$

where $I$ is the mean excitation energy, $T_{\max }=\frac{2 m_{e} c^{2} \beta^{2} \gamma^{2}}{1+2 \gamma m_{e} / M+\left(m_{e} / M\right)^{2}}$ is the maximum kinetic energy possible to transfer to a free electron and $\delta(\beta \gamma)$ is a dielectric screening correction. The other constants and terms are defined in [79] and material properties and methods to calculate $\delta(\beta \gamma)$ using fitted data were available in [80]. $-\frac{d E}{d x}$ was calculated for a range of proton momenta for lead, aluminium, iron and the organic scintillator (E093) used in BigBite and the values agree well with the PDG results (see fig. 3.22). 


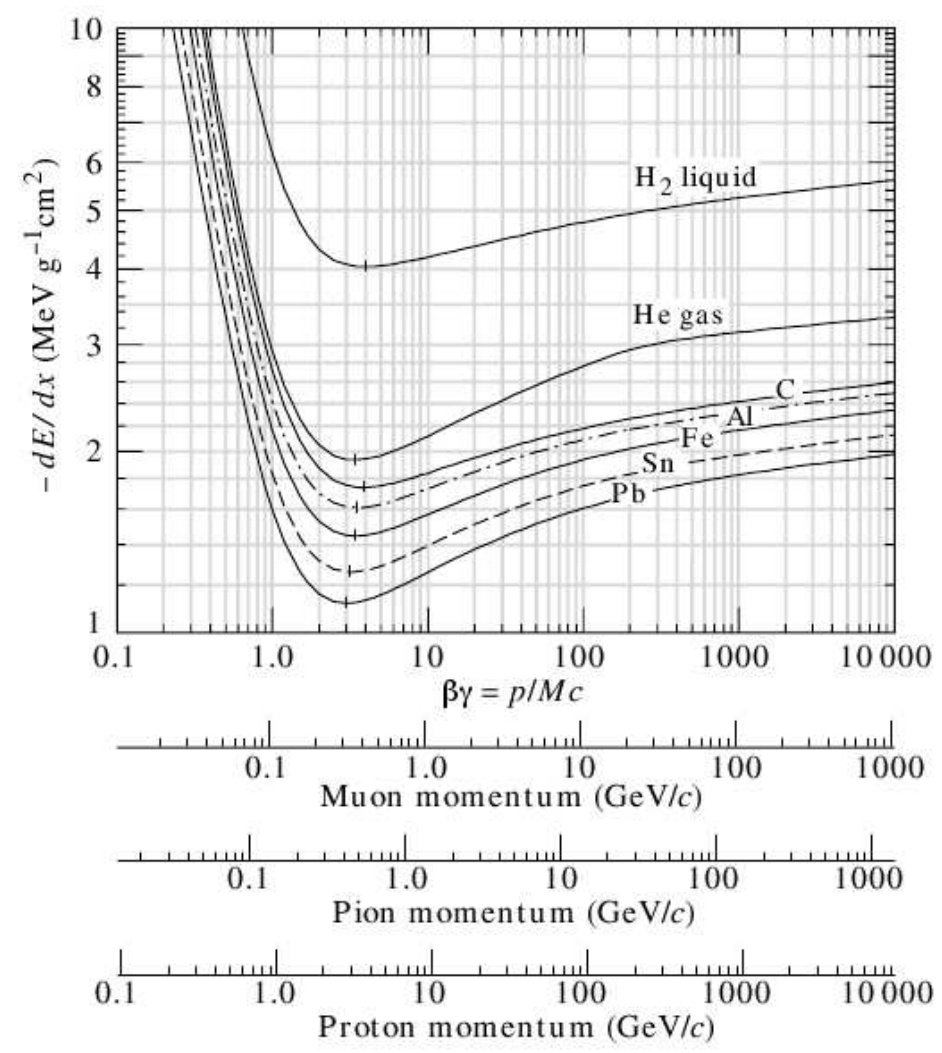

Figure 3.22: $-\frac{d E}{d x}$ calculated as a function of momentum for different particles for various materials in the Particle Data Group (PDG) booklet.

The energy deposited in the $\mathrm{dE}$ (3 $\mathrm{mm}$ thick) and $\mathrm{E}(30 \mathrm{~mm}$ thick) scintillator bars was calculated using $E_{d e p}=-\frac{d E}{d x} \rho l$, where $\rho$ is the density of the scintillator and $l$ is a discrete step distance $(0.2 \mathrm{~mm})$ that the particle transverses through the medium. This small value of deposited energy was subtracted from the energy of the particle and its momentum, $\beta$ and $\gamma$ were updated. This process was repeated until the particle had come to rest or had broken through both layers of scintillator. The calculated $\mathrm{dE}$ and E bar energy deposited (see fig. 3.23) was in good agreement with the Geant4 simulation (see section 3.4.5). The coefficients to convert the ADC channel numbers to $\mathrm{MeV}$ were calculated to produce the same breakthrough points as this simulation. 

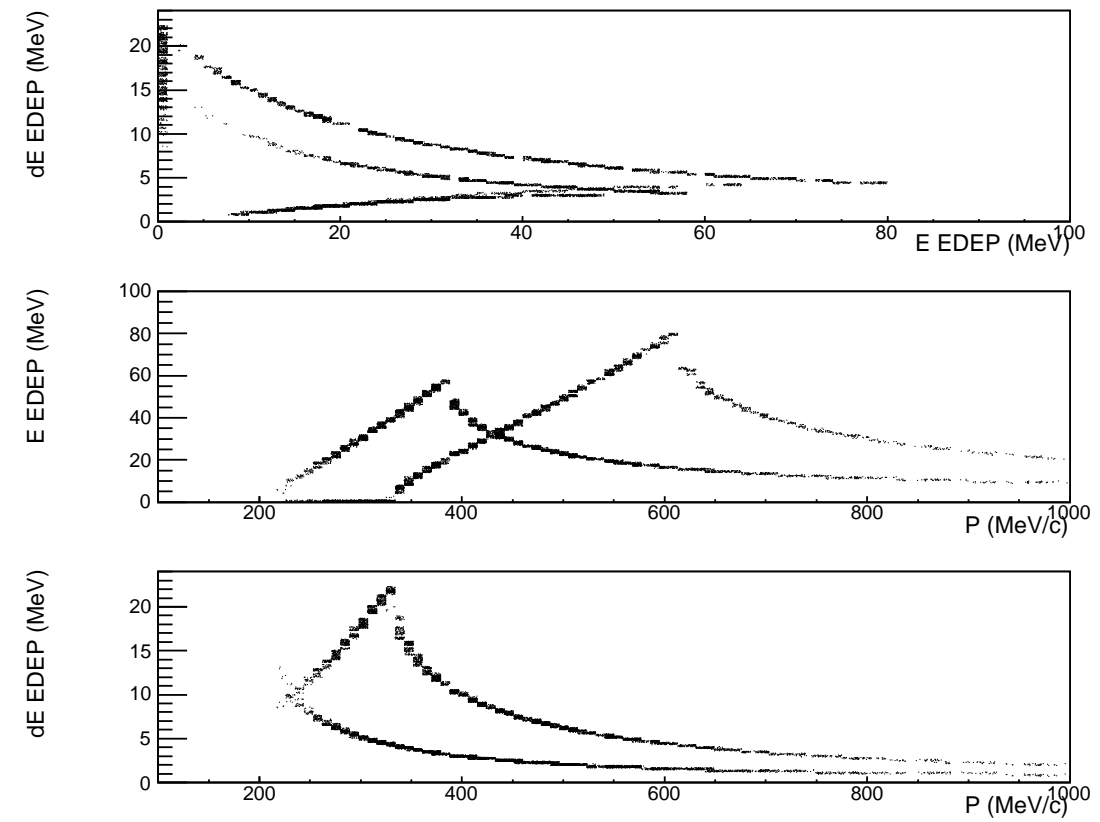

Figure 3.23: Top: Calculated dE bar energy deposited against the calculated E bar energy deposited for protons and deuterons. Middle: Calculated E bar energy deposited plotted as a function of initial momentum for protons and deuterons. Bottom: Calculated dE bar energy deposited plotted as a function of initial momentum for protons and deuterons.

The separation of protons and deuterons is more clear in the plots of energy deposited in the $\mathrm{dE}$ and $\mathrm{E}$ bars displayed as a function of momentum compared to the $\mathrm{dE}$ vs E bar energy deposited distribution (see fig. 3.23). This separation of protons and deuterons is also expressed in fig. 3.24. $2 \mathrm{D}$ cuts were used to select the high and low momentum deuterons on the right hand plots. These graphical cuts were used together with a cut on calculated energy deposited for the deuteron in the $\mathrm{E}$ bar, to ensure momentum measured in BigBite allows the deuteron to have enough energy to reach the E scintillator plane, to form an overall deuteron PID cut. This cut applied to dE vs E distribution removes protons and pions to leave only deuterons remaining (see fig 3.25). 

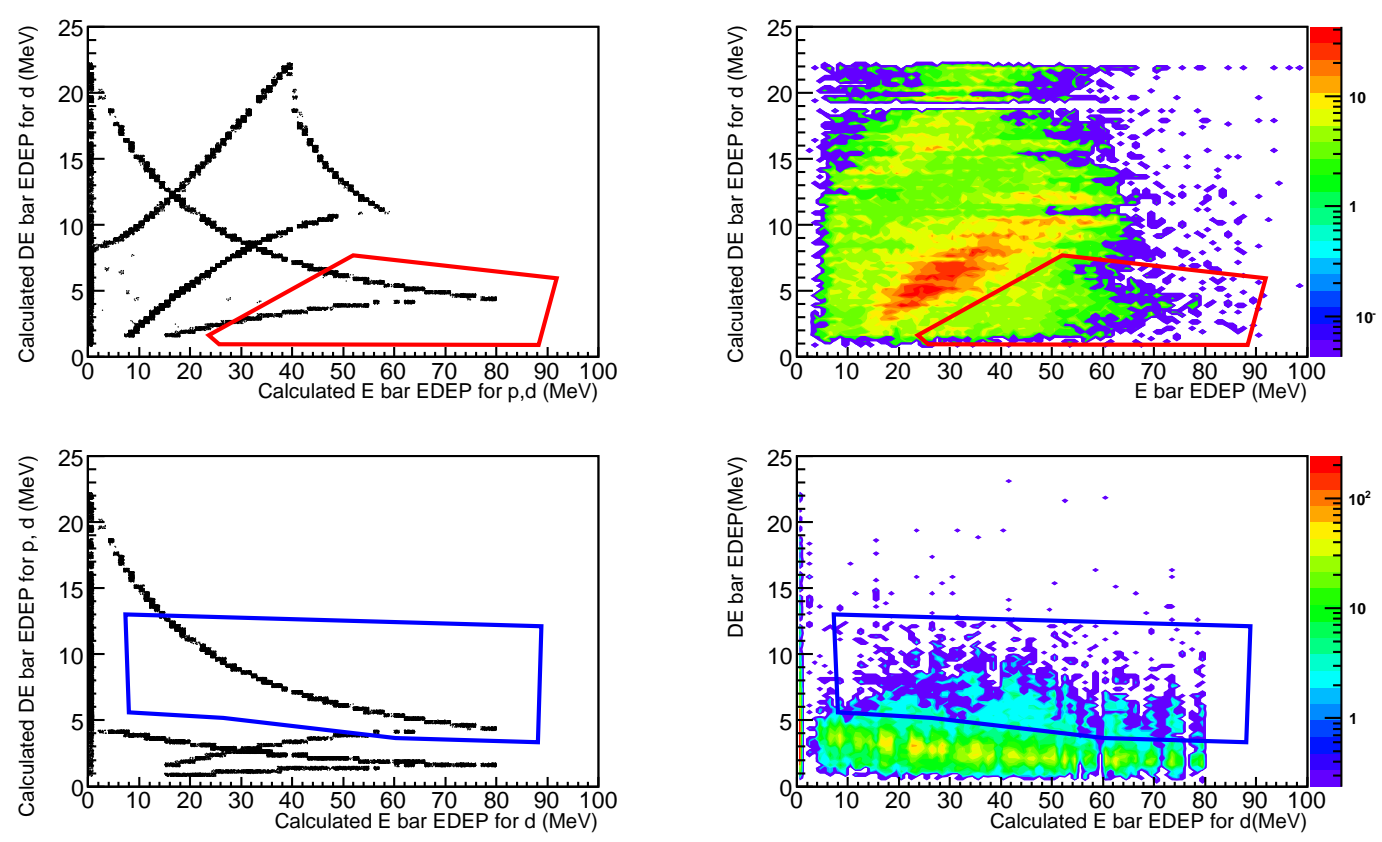

Figure 3.24: Top-left: Calculated dE energy for deuterons plotted against E bar energy calculated for protons and deuterons. This shows the calculated $\mathrm{dE}$ vs E energy deposited for deuterons, as in figure 3.23 and also the calculated $\mathrm{dE}$ values for deuterons plotted against the calculated $E$ values for protons of the same momentum. The area enclosed in the red graphical cut shows that the deuterons of greater momentum than the breakthrough point $(565 \mathrm{MeV})$ are cleanly separated from the protons. Top-right: Measured E bar energy plotted against the calculated $\mathrm{dE}$ bar energy for deuterons. A graphical cut similar to the previous red graphical cut, was used on this histogram to identify the deuterons of high momentum. The cut cannot extend any further to include the highest distribution of deuteron momentum $(>820 \mathrm{MeV} / \mathrm{c})$ due to the high concentration of protons in this area. These missing deuterons have a greater momentum than the upper limit $(650 \mathrm{MeV} / \mathrm{c})$ of ${ }^{12} \mathrm{C}\left(e, e^{\prime} p\right)$ missing momentum in the CK3 kinematics. Bottom-left: The calculated dE energy for deuterons and protons plotted against E bar energy calculated for deuterons. The area enclosed in the blue graphical cut shows that the deuterons of lesser momentum than the breakthrough point are cleanly separated from the protons. BottomRight: The measured dE bar energy plotted against the calculated E bar energy for deuterons. A graphical cut similar to the previous blue graphical cut, was used on this histogram to identify the deuterons of lower momentum than the breakthrough point. 


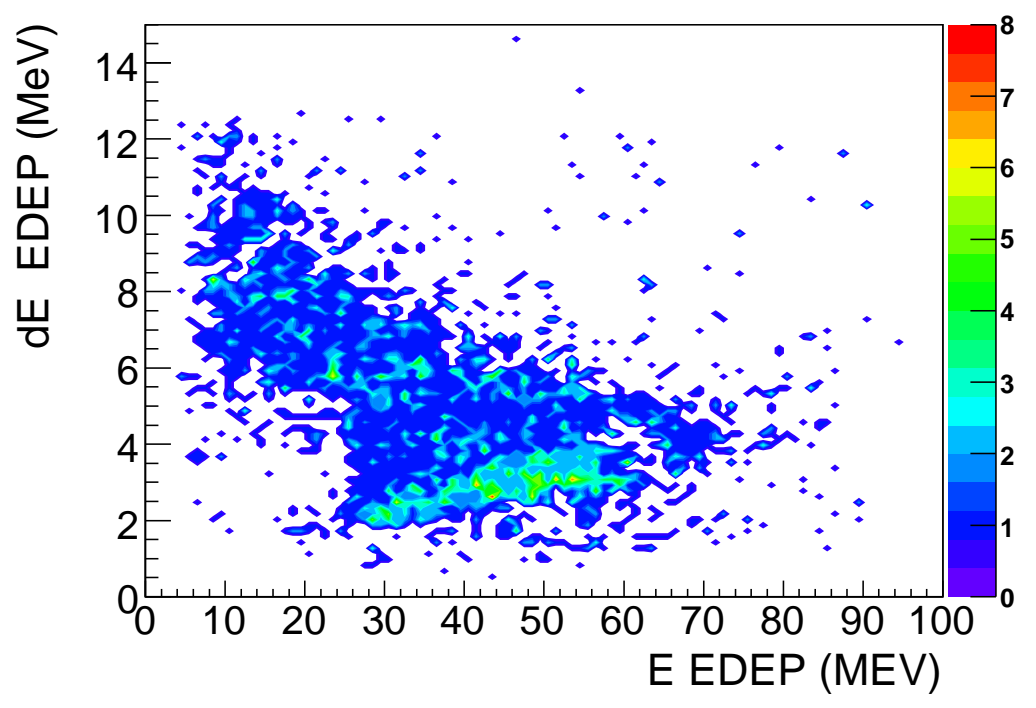

Figure 3.25: dE bar energy deposited versus E bar energy deposited with deuteron cuts applied for the CK2 kinematics of $p_{\text {miss }}=400 \mathrm{MeV}$ for the ${ }^{12} \mathrm{C}\left(e, e^{\prime} p\right)$ reaction.

\subsubsection{Yield}

To obtain the yields of the ${ }^{12} C\left(e, e^{\prime} p p\right)$ and ${ }^{12} C\left(e, e^{\prime} p d\right)$ reactions at the different experimental kinematic settings, the calculated TOF of the proton or deuteron from the target to the E plane in the BigBite spectrometer was subtracted from the measured TOF. Two TOFs were calculated for BigBite events, derived from the measured momentum and particle path length in BigBite and the corresponding velocities for both protons or deuterons. By subtracting either the calculated TOF for protons or deuterons from the measured TOF, the real protons or deuterons are distributed within a peak centred around $0 \mathrm{~ns}$.

\section{Final Cut Selection}

The TOF minus the calculated TOF for the ${ }^{12} C\left(e, e^{\prime} p p\right)$ and ${ }^{12} C\left(e, e^{\prime} p d\right)$ reactions for the three kinematic settings of missing momenta for the ${ }^{12} C\left(e, e^{\prime} p\right)$ reaction of 300 (CK1), $400(\mathrm{CK} 2)$ and 500 (CK3) $\mathrm{MeV} / \mathrm{c}$ (top to bottom) are shown in figures 3.26 and 3.27 respectively. For both reactions, the cuts used to obtain the ${ }^{12} C\left(e, e^{\prime} p\right)$ reaction as previously discussed earlier in the chapter have been applied as well as the BigBite cuts, namely the initial cuts discussed in section 3.4.6, which remove all of the background pion contamination. The kinematic cuts to remove the $\Delta$ excitations discussed in section 3.3 .1 were applied for both reactions. These kinematic cuts are designed to enhance the short 
correlation signal which is of more interest in the ${ }^{12} C\left(e, e^{\prime} p p\right)$ reaction, but they also reduced background without affecting the signal in the ${ }^{12} C\left(e, e^{\prime} p d\right)$ reaction.

The TOF minus calculated TOF for the ${ }^{12} C\left(e, e^{\prime} p p\right)$ reaction show clear peaks centred around the $0 \mathrm{~ns}$ for all three kinematics. There are no further specific cuts to enhance the proton signal by removing deuterons, as they are at such low levels compared to the protons. However the ${ }^{12} C\left(e, e^{\prime} p d\right)$ reaction (see fig. 3.27) shows that the deuteron signal is washed out almost completely by the dominant protons in the background for the CK1 and CK2 kinematic settings. For the CK3 setting though, there are two peaks at $-17 \mathrm{~ns}$ and $0 \mathrm{~ns}$ corresponding to protons and deuterons respectively.

The application of the deuteron cuts, discussed in section 3.4.6, to the TOF minus calculated TOF plots for the ${ }^{12} C\left(e, e^{\prime} p d\right)$ reaction, removes the protons but the deuteron signal of the CK1 and CK3 kinematics becomes very weak (see fig. 3.28). The deuteron signal for the ${ }^{12} C\left(e, e^{\prime} p d\right)$ reaction at the CK1 kinematics is weak most likely due to the low missing momenta of $300 \mathrm{MeV} / \mathrm{c}$ of the ${ }^{12} \mathrm{C}\left(e, e^{\prime} p\right)$ reaction. There is a noticeable deuteron peak at $0 \mathrm{~ns}$ for the CK2 kinematics. There are not enough events for the CK3 kinematics with the deuteron PID cuts for there to be a strong deuteron peak.

To calculate yields for the ${ }^{12} C\left(e, e^{\prime} p d\right)$ reaction for the CK3 kinematics, the TOF minus calculated TOF without the specific deuteron cuts was used because the deuteron peak is much more prominent than with the deuteron cuts. The deuteron cuts work much better for the CK2 kinematics and yield was calculated using the TOF minus calculated TOF with the deuteron cuts. For the CK1 kinematics, there is no noticeable deuteron signal in the TOF minus calculated TOF without deuteron cuts, therefore yield extraction was performed with the deuteron cuts in order to obtain a rough upper limit. 

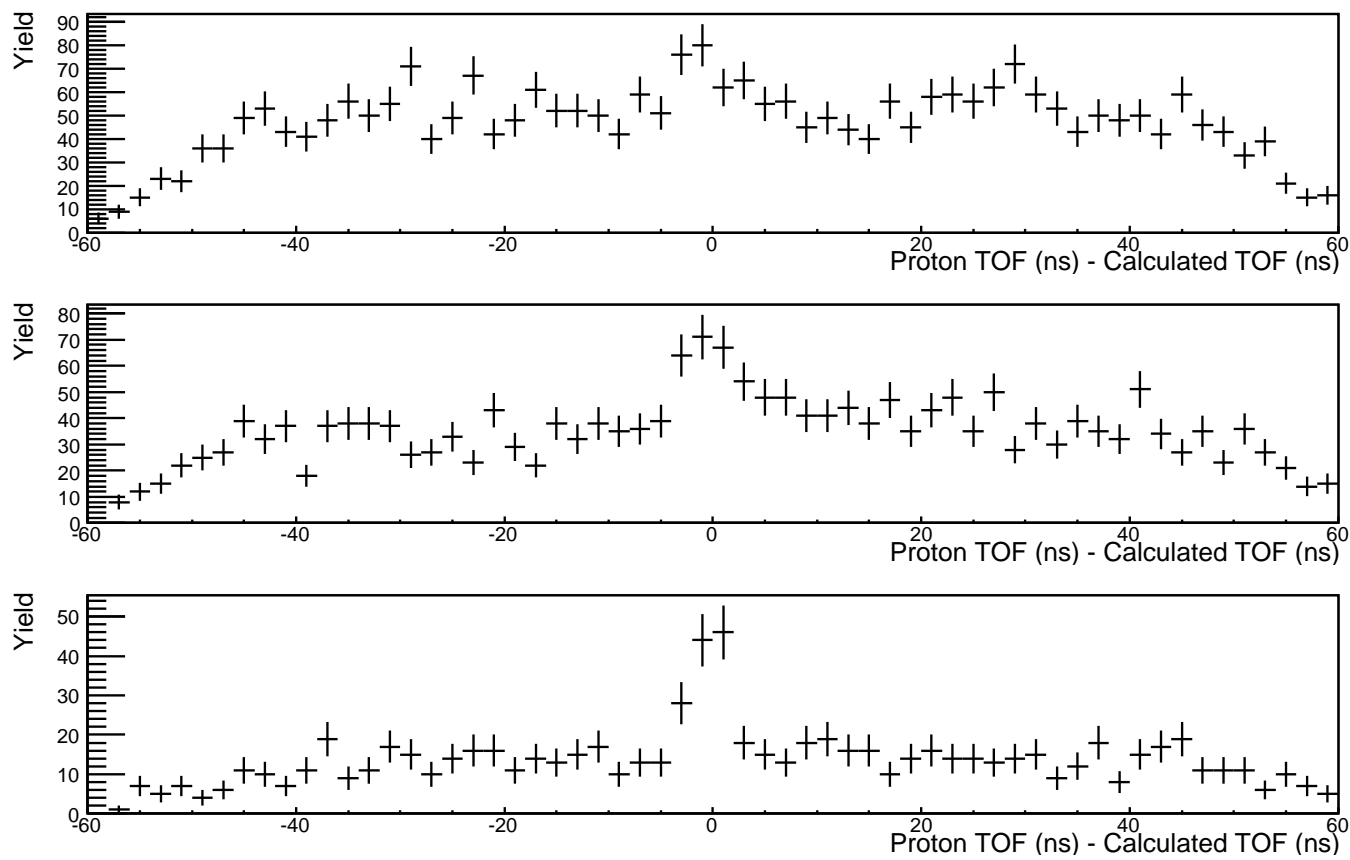

Figure 3.26: Measured TOF minus calculated TOF of the ${ }^{12} C\left(e, e^{\prime} p p\right)$ reaction for ${ }^{12} C\left(e, e^{\prime} p\right)$ missing momenta of 300 (CK1), 400 (CK2) and 500 (CK3) MeV/c (top to bottom) with no specific BigBite proton cuts.
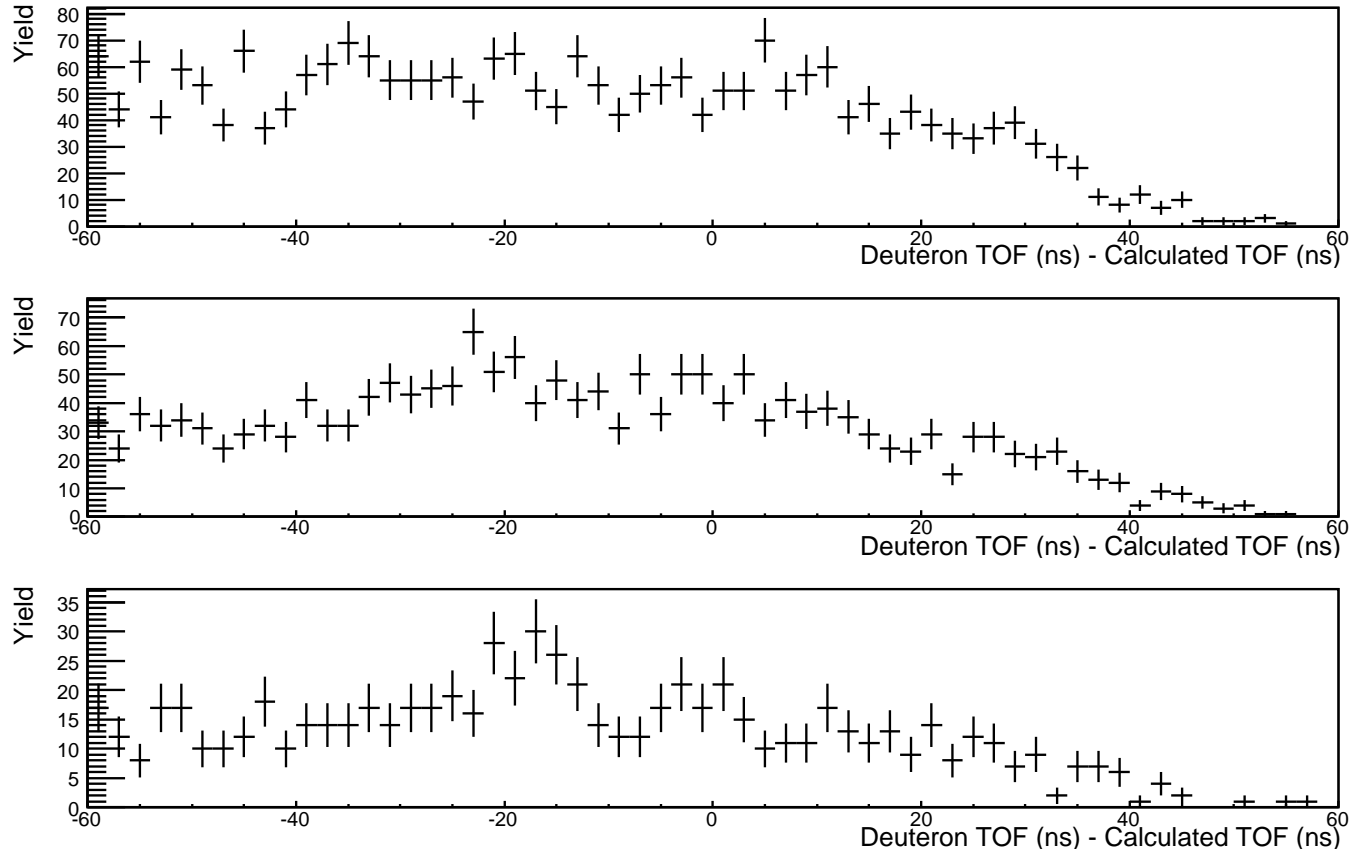

Figure 3.27: Measured TOF minus calculated TOF of the ${ }^{12} C\left(e, e^{\prime} p d\right)$ reaction for ${ }^{12} \mathrm{C}\left(e, e^{\prime} p\right)$ missing momenta of 300 (CK1), 400 (CK2) and 500 (CK3) $\mathrm{MeV} / \mathrm{c}$ (top to bottom) with no specific BigBite deuteron cuts. 

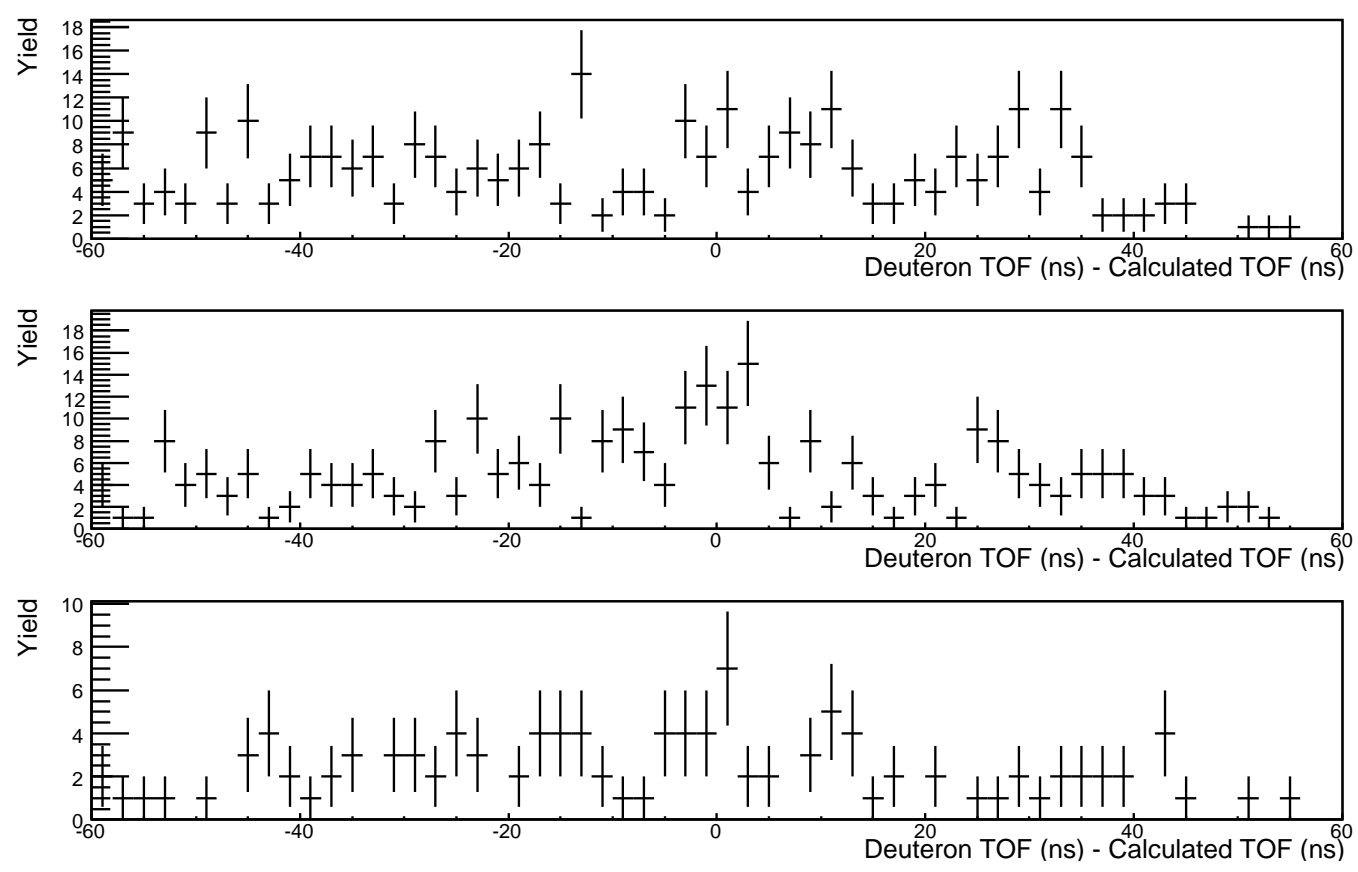

Figure 3.28: Measured TOF minus calculated TOF of the ${ }^{12} C\left(e, e^{\prime} p d\right)$ reaction for ${ }^{12} C\left(e, e^{\prime} p\right)$ missing momenta of 300 (CK1), 400 (CK2) and 500 (CK3) MeV/c (top to bottom) with BigBite deuteron cuts.

\section{Fitting Methods and Yield Extraction}

This analysis will focus on the extraction of the ${ }^{12} C\left(e, e^{\prime} p d\right)$ reaction as the ${ }^{12} C\left(e, e^{\prime} p p\right)$ reaction has already been analysed by Ran Shneor and published [68]. The difficulties in extracting a meaningful yield calculation for ${ }^{12} C\left(e, e^{\prime} p d\right)$ reaction are very apparent when inspecting the TOF difference plots for the different kinematics in the previous figures of 3.27 and 3.28. The Geant 4 simulation (see section 3.4.5) showed that the deuteron widths should be roughly twice that of the protons and that the there should only be broad similarities in the peaks across the different kinematics but with some unpredictable variations in width and peak centre location. Using this information was important when selecting between the methods for obtaining the yield and between different fitting methods. This means that the widths of any Gaussian fits should not be fixed when applied to the TOF minus calculated TOF of the different kinematic settings and the centre of the Gaussian fits should be allowed some freedom to vary slightly from zero. 


\section{Fitting Peak \& Background}

The first method used to extract yields from the TOF minus calculated TOF spectra was to fit a flat background and also to fit a function with the same flat background and a Gaussian fit to the deuteron peak for the CK1 and CK2 kinematics (see 3.29). The yield was then calculated by finding the difference in integrals between the two fits over the same range. However this method needed adjusting for the CK3 kinematics, because the TOF minus calculated TOF spectrum for these kinematics also included a proton peak to the left of the deuteron peak. This is because no specific deuteron cuts for BigBite event selection were used for CK3, but were applied to the TOF difference spectrums of CK1 and CK2. To account for the proton peak, an additional Gaussian fit to the proton peak was incorporated into the fit of the background and also into the fit of the deuteron peak and background.

The quality of the fits to the CK1 and CK2 TOF difference spectrums are not great due to the low statistics and very small cross section of the ${ }^{12} C\left(e, e^{\prime} p d\right)$ reaction at these kinematics. The Gaussian fit for the CK1 kinematics is particularly questionable due to the number of events to the immediate right well above the background fit and with a similar magnitude to the events within the peak. The Gaussian peak also only fits well with three points and neglects two lower points within the range of the Gaussian fit. The yield of 29.29 events derived from this fit is best described as a crude estimate of the upper limit of ${ }^{12} C\left(e, e^{\prime} p d\right)$ events.

For the CK2 kinematics, there is a noticeable deuteron peak rising above the background and the Gaussian fit is much more acceptable than for the CK1 kinematics. However the flat background fit to the left of the peak is on the low side. The low number of events and the ragged nature of the background make it impossible to fit any kind of convincing fit function, including linear background fits. The yield for the ${ }^{12} C\left(e, e^{\prime} p d\right)$ CK2 kinematics for this fitting method is 37.475. The Gaussian and background fits for the CK3 fits are good due to the higher number of events and the yield for this method is 40.33 . 

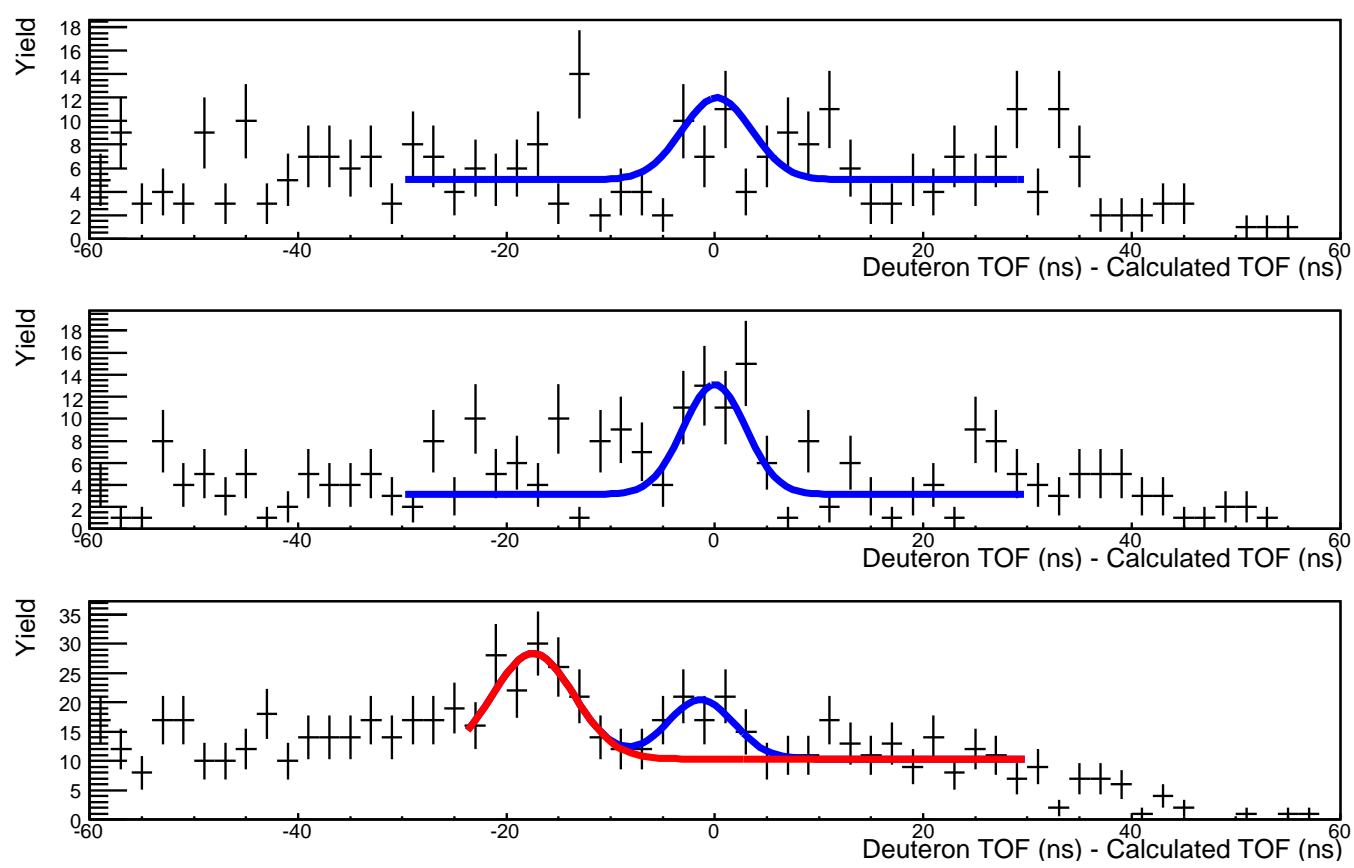

Figure 3.29: Measured TOF minus calculated TOF for ${ }^{12} C\left(e, e^{\prime} p\right)$ missing momenta of 300 (CK1), 400 (CK2) and 500 (CK3) $\mathrm{MeV} / \mathrm{c}$ (top to bottom). For CK1 and CK2 kinematics, the TOF difference spectrums are fitted with Gaussian superimposed on a flat background (from 0 to $60 \mathrm{~ns}$ ). For CK3, there is an additional proton peak to the left of the deuteron peak in the centre. A Gaussian fit of this proton peak is included in the fit to the background and the fit of the background + deuteron peak.

\section{Parse Random Background Subtraction Method}

Another method to calculate the yields was to simply integrate the deuteron peaks of TOF difference spectrums and also either side of the peak. From the number of background events either side of the peak, the average number of background events per bin within the peak region was calculated. The number of background events within the peak region is calculated using this average number and the number of bins within the peak region. The yield is then obtained from the integral of the peak region minus the number of background events. This method is crude but it is effective when the application of other fitting methods are difficult, as in fits for the CK1 and CK2 kinematics. Therefore, this method was used as the primary method for extracting the yields and the Gaussian and flat background fitting method was used to estimate the systematic uncertainties of the yield calculation.

The peak region $\left(P_{R}\right)$ was defined as $-7 n s<P_{R}<7 n s$ and the upper $\left(B_{U R}\right)$ and lower $\left(B_{L R}\right)$ background sampling regions as $7 n s<B_{U R}<30 n s$ 
and $-30 n s<B_{L R}<-7 n s$. These boundaries coincide with the limits of the background fits and of the general location of where the Gaussian peak fits joined the background fits. The additional proton peak to the left of the deuteron peak for the CK3 kinematics, meant that the lower background sampling region could not be used. The background estimate in the peak region for CK3 is therefore derived only from the upper background sample region. The results for both yield extraction methods are shown in table 3.2 with the statistical and systematic errors which are discussed in the next section.

\section{Systematic Errors and Yield Results}

The yield results for the parse random background subtraction and for the flat background and Gaussian fit method are shown in table 3.2, with the statistical errors (square root of the yield) for both. The parse random background subtraction method was chosen to ascertain the final yield results due to the difficulty in obtaining quality of fits for the other yield extraction method. The CK3 kinematic setting where the quality of the fitting method was good agrees quite closely with the result from the parse random background subtraction method. For the CK2 kinematics, the fitting method produces a higher the yield due to the background fit being lower than the average background count per bin. This was due to the background being ragged and the fitting routine favouring the lower points because of their heavier weighting compared to the higher points (points have a greater statistical error $(\sqrt{n})$ with the more counts $(n))$. The yields for the CK1 kinematics are wildly different with the yield from the fitting method being about 4 times as large as the yield from the parse random background subtraction method. The yield for the CK1 spectrum is low compared to the other kinematics and the application of the fitting method was difficult. Many of the attempts of tweaking the fit to find something reasonable resulted in a failed fit or with a Gaussian fit to one point. The final fit was the most reasonable.

The parse random subtraction method is better for extracting a yield for these low statistic plots compared to fitting, but it does not take account of the statistical error of the points and the location of sampling region boundaries can vary the results within the statistical error. The systematic errors of the yield extraction were calculated by taking the magnitude of the difference between the yield results of the parse random background subtraction and the fitting method (see table 3.2). Therefore the systematic error acknowledges the differences of the different yield extraction methods and the problems of the parse random 
background subtraction method.

The CK3 kinematics have the least systematic errors because two different yield extraction methods obtained similar results, while the systematic errors for the CK1 kinematics are much larger due the large disagreement in yield extraction results. The systematic errors also reflect the quality of the deuteron peaks in the TOF difference plots. The most obvious deuteron peak is in the CK3 kinematics and it has the highest yield and lowest systematic error. Conversely, the least obvious deuteron peak is in the CK1 kinematics and it has the lowest yield and highest systematic error.

\begin{tabular}{|c|c|c|}
\hline Kinematics & Yield & Fit Yield \\
\hline \hline CK1 & $7.20 \pm 2.68 \pm 22.09$ & $29.29 \pm 5.47$ \\
\hline CK2 & $28.73 \pm 5.36 \pm 8.75$ & $37.48 \pm 6.12$ \\
\hline CK3 & $38.83 \pm 6.23 \pm 1.50$ & $40.33 \pm 6.35$ \\
\hline
\end{tabular}

Table 3.2: Yield results using the parse random subtraction method and the yield using a flat background and Gaussian fits of the ${ }^{12} C\left(e, e^{\prime} p d\right)$ reaction for each kinematic setting with statistical error first and then systematic error on the final yield results. 


\section{Chapter 4}

\section{Simulation and Acceptance Correction}

\section{$4.1 \quad(e, e ’ p d)$ Simulation}

To find the experimental acceptance, a root based simulation of the experiment in Hall A was written using a simplified phase space model of the ${ }^{12} C\left(e, e^{\prime} p d\right)$ reaction. The simulation consisted of 100 million events for each of the three kinematics settings.

\subsubsection{Event Generator}

Each event was generated by randomly selecting an electron event from real data using a 3D histogram of the Left HRS acceptance variables of $\delta, y_{t g t}$ and $\phi_{t g t}$ (see section 3.2.2). $\theta_{t g t}$ was reconstructed from the a linear fit between $\theta_{t g t}$ and $y_{\text {tgt }}$. The generated electron parameters were scaled by a factor of 1.3 (except $\left.y_{\text {tgt }}\right)$ to ensure the generated electron acceptance was greater than the physical acceptance of the left HRS. The beam-target interaction point was calculated from $y_{\text {tgt }}$ (the horizontal distance along the target in the HRS reference frame). The $0.25 \mathrm{~mm}$ thick carbon target was slanted at $20^{\circ}$ to the beam of $4.627 \mathrm{GeV}$.

The reaction mechanism consisted of an electron scattering off a quasi-free ${ }^{3} \mathrm{He}$ target with an initial Fermi momentum $\left(p_{f}\right)$ inside a ${ }^{12} \mathrm{C}$ nucleus, with ${ }^{9} \mathrm{Be}$ acting as a spectator. The ${ }^{9} \mathrm{Be}$ spectator is moving in a back-to-back direction to the ${ }^{3} \mathrm{He}$ target with equal momentum and was put on its mass-shell, by calculating its energy $E_{\text {spec }}=\sqrt{p_{f}^{2}+M_{\text {Spec }}^{2}}$, where $M_{\text {Spec }}$ is the mass of the ${ }^{9} B e$. The energy of the ${ }^{3} \mathrm{He}$ target was calculated using $E_{t g}=M_{C 12}-E_{\text {spec }}$ and the Lorentz vector 
of the intermediate reaction product of the struck ${ }^{3} H e^{*}$ target was calculated by adding together the Lorentz vectors of initial ${ }^{3} \mathrm{He}$ target and the virtual photon $\vec{q}=\vec{e}-\overrightarrow{e^{\prime}}$, with the invariant mass of ${ }^{3} H e^{*}$ required to be greater than zero to ensure consistent results.

The struck ${ }^{3} H e^{*}$ target was Lorentz boosted to its centre of mass in order to calculate the kinematics of its back-to-back break-up into a proton and deuteron. The decay energy of the proton was calculated using $E_{\text {Proton }}=\frac{M_{\text {InvParent }}^{2}+m_{\text {proton }}^{2}-m_{\text {deuteron }}^{2}}{2 M_{\text {InvParent }}}$, where $M_{\text {InvParent }}$ is the invariant mass of ${ }^{3} H e^{*}$. The decay momentum of both the proton and deuteron was calculated using $p_{\text {dec }}=\sqrt{E_{\text {proton }}^{2}-m_{\text {proton }}^{2}}$, with $E_{\text {Proton }}$ and $p_{\text {dec }}$ required to be greater than zero. The deuteron energy was calculated using $E_{\text {deuteron }}=\sqrt{p_{\text {dec }}^{2}+m_{\text {deuteron }}^{2}}$ and the initial Lorentz vectors of the proton and deuteron were generated using angles sampled randomly from an isotropic distribution and then Lorentz boosted into the lab frame.

\subsubsection{Extraction of Simulated Data}

Simulated data was created from generated events by accounting for the resolution and acceptance of the HRS and BigBite spectrometers and the energy loss of the particles. The energy loss of protons and deuterons was calculated using the same method as in section 3.4.6, however the energy loss for the scattered electron was calculated using the electron Bethe-Bloch formula, see [79]. The acceptance and resolution characteristics for the HRS and BigBite spectrometers used in the simulation are shown in table 4.1. 2D cuts on generated particle's vertical and horizontal angles versus its momentum and each other, were obtained from real data to correct for the inter-dependencies of these parameters on the real acceptance of the spectrometers (see fig. 4.1). The spectrometer output variables were folded with the detector resolutions using Gaussians and the resolution values in table 4.1 . 


\begin{tabular}{|c|c|}
\hline HRS Momentum range & $0.3-4.0 \mathrm{GeV} / \mathrm{c}$ \\
\hline \hline HRS Momentum acceptance & $-4.5 \%<\delta p / p<+4.5 \%$ \\
\hline HRS Momentum Resolution & $1 \times 10^{-4}$ \\
\hline HRS Angular Range: Left, Right & $12.5-130^{\circ}, 150^{\circ}$ \\
\hline HRS Horizontal Acceptance & $\pm 30 \mathrm{mrad}$ \\
\hline HRS Vertical Acceptance & $\pm 60 \mathrm{mrad}$ \\
\hline HRS Horizontal Resolution & $0.5 \mathrm{mrad}$ \\
\hline HRS Vertical Resolution & $1.0 \mathrm{mrad}$ \\
\hline BB Momentum Range & $0.2-1.0 \mathrm{GeV} / \mathrm{c}$ \\
\hline BB Momentum Resolution & $25 \mathrm{MeV} / \mathrm{c}$ \\
\hline BB Horizontal Acceptance & $\pm 80 \mathrm{mrad}$ \\
\hline BB Vertical Acceptance & $-0.37 \mathrm{to} 0.44 \mathrm{rad}$ \\
\hline BB Horizontal Resolution & $15 \mathrm{mrad}$ \\
\hline BB Vertical Resolution & $15 \mathrm{mrad}$ \\
\hline
\end{tabular}

Table 4.1: Resolution and acceptance characteristics of the HRS and BigBite spectrometers.
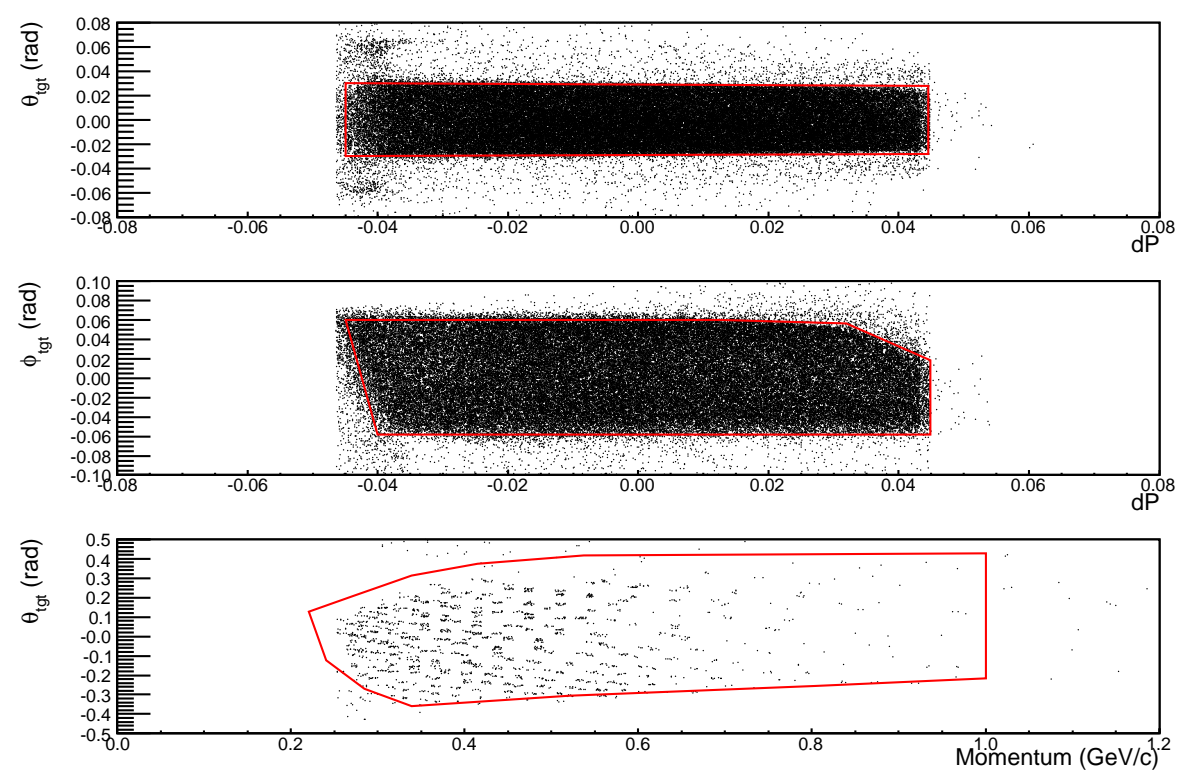

Figure 4.1: Graphical acceptance cuts obtained from real data used in simulation. Top: Horizontal angle versus fractional momentum for Right HRS. Middle: Vertical angle versus fractional momentum of Right HRS. Bottom: Vertical angle vs momentum measured in BigBite. 


\subsubsection{Comparison of Generated, Simulated and Experimen- tal Data}

The event generator and simulation results were compared with experimental results for one million generated events, to see how well they agree and highlight any problems. The momentum, displacement in the dispersive (vertical) direction from the central trajectory $\left(y_{t g t}\right)$ and the vertical $\left(\theta_{t g t}\right)$ and horizontal $\left(\phi_{t g t}\right)$ angles in the same plane relative to the central trajectory of the generated particles and simulated spectrometer detector measurements are compared to experimental data in figures $4.2,4.3$ and 4.4 .
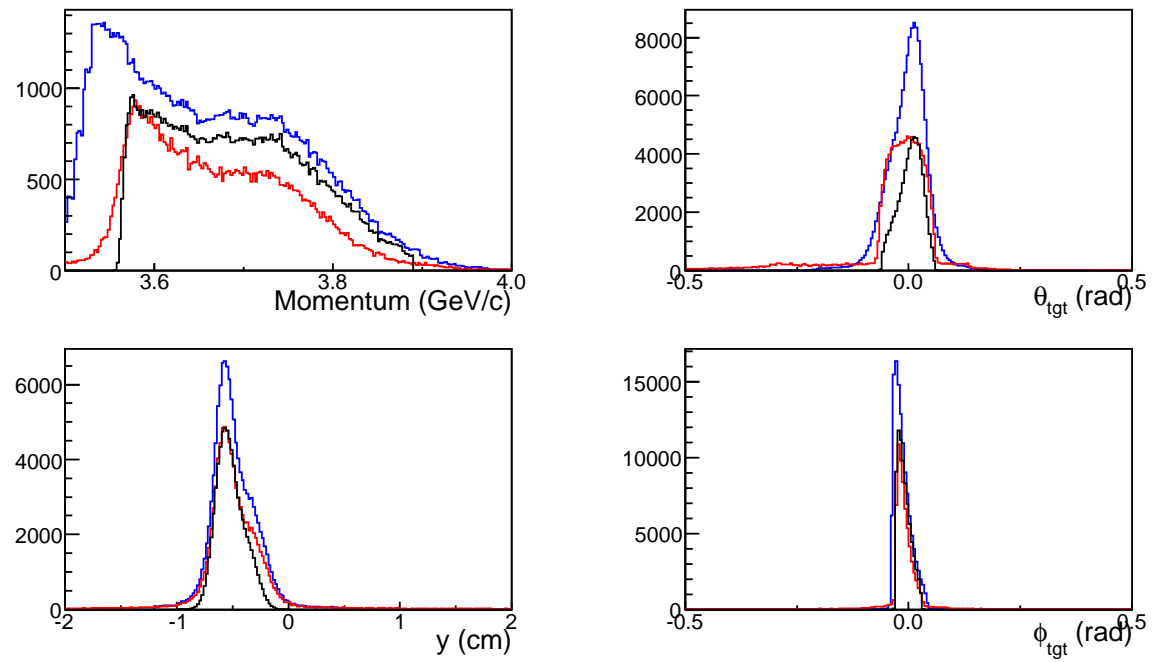

Figure 4.2: Comparison of the left HRS experimental data (red), the generated scattered electron data (blue) and simulated left HRS detected electron data (black). Top left: Momentum $(G e V / c)$. Top right: $\theta_{t g t}(\mathrm{rad})$, vertical angle. Bottom left: $y_{\text {tgt }}$ position of reaction vertex. Bottom right: $\phi_{t g t}(\mathrm{rad})$, horizontal angle. All plots are scaled to fit on the histograms. 

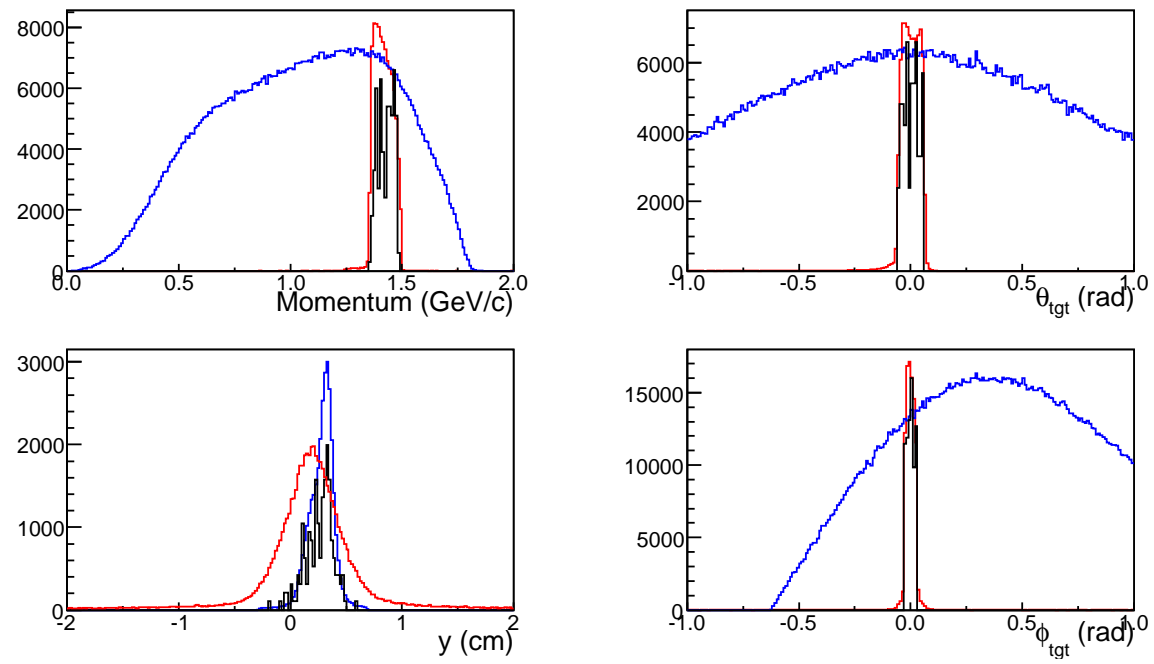

Figure 4.3: Comparison of the right HRS experimental data (red), the generated proton data (blue) and simulated right HRS detected proton data (black). Top left: Momentum $(\mathrm{GeV} / \mathrm{c})$. Top right: $\theta_{t g t}(\mathrm{rad})$, vertical angle. Bottom left: $y_{t g t}$ position of reaction vertex. Bottom right: $\phi_{t g t}(\mathrm{rad})$, horizontal angle. All plots are scaled to fit on the histograms.
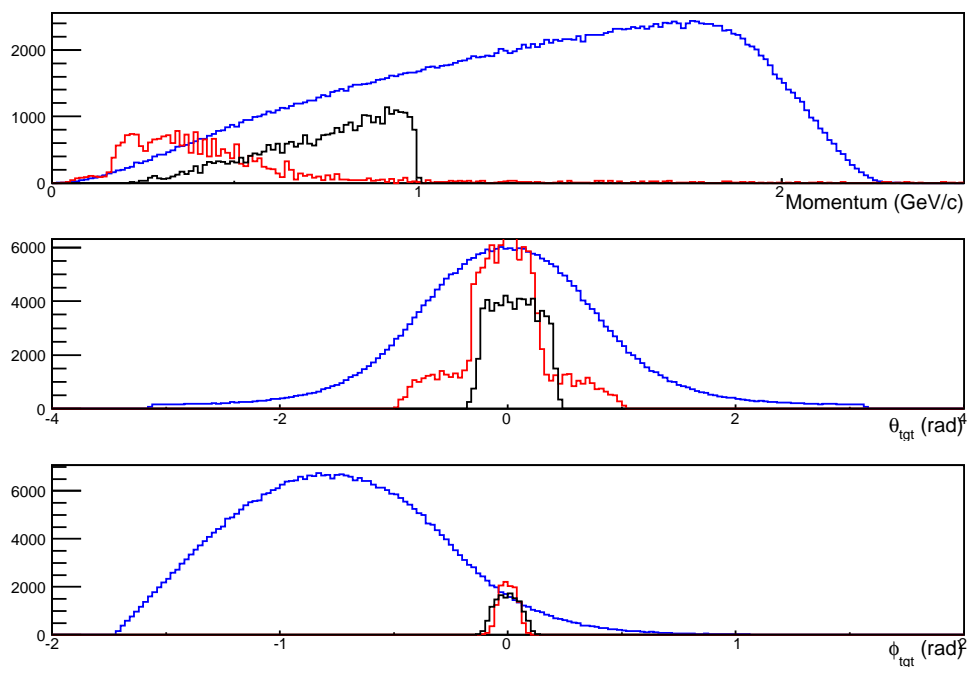

Figure 4.4: Comparison of the BigBite experimental data (red), the generated deuteron data (blue) and simulated BigBite detected deuteron data (black). Top: Momentum $(\mathrm{GeV} / \mathrm{c})$. Middle: $\theta_{t g t}(\mathrm{rad})$, vertical angle. Bottom: $\phi_{t g t}(\mathrm{rad})$, horizontal angle. All plots are scaled to fit on the histograms. BigBite does not measure the reaction vertex position and assumes it is at centre of target.

The simulated detector response agrees quite well with the experimental data and this gives confidence in the performance of the simulation. The generated and simulated deuterons for BigBite have a different momentum distribution than 
the experimental data because the experimental data contains mostly protons. The momentum spectrum of the simulated deuterons in BigBite also rises until the momentum acceptance cut takes affect. The real momentum spectrum of deuterons in BigBite in the simulation is different when the coincidence of the scattered electron and proton in the left and right HRS is required. These plots also illustrate the momentum and angular range over which the events are generated given the kinematics of the ${ }^{12} C\left(e, e^{\prime} p d\right)$ reaction. For the right HRS and BigBite detectors only a small fraction of the generated events for protons and deuterons respectively are within the acceptance range of each detector. This shows that the experimental acceptance is very small and is discussed in the next section.

\subsubsection{Acceptance Correction}

The acceptance of a detector is the fraction of events of a reaction that produce a particle that is measured and recorded compared to the many events that are not. In order for a detector to measure a particle, the particle must have a trajectory from the target that corresponds to the physical opening of the detector. Also the momentum of the particle must fall within the momentum range of the detector. To extract the cross section from the measured yield of the experiment, the acceptance of all the detectors have to be taken into account. Therefore acceptance correction factors for each kinematic setting are used to adjust the measured yield of the experiment to account for the limited acceptance of the detectors used. The purpose of the simulation was to obtain a value for the total acceptance for the ${ }^{12} C\left(e, e^{\prime} p d\right)$ reaction by counting the number of events that simultaneously produced electrons in the left HRS, protons in the right HRS and deuterons in BigBite for the total number of generated events. The acceptance was calculated using the following equation:

$$
A c c=\frac{N_{\text {sim }} \Omega_{\text {gen }}}{N_{\text {gen }}}
$$

where, $N_{\text {gen }}$ are the number of generated events, $N_{\text {sim }}$ is the number of simulated events and $\Omega_{\text {gen }}$ is the solid angle of the generated electron. Since real data was used to produce the scattered electron, $\Omega_{\text {gen }}$ is equal to the normal solid angle of $7.2 \mathrm{msr}$ of the left HRS multiplied by the square of the factor of 1.3 used to scale this real data, to increase the generated solid angle to be greater than the left HRS solid angle acceptance. 
To estimate the systematic errors relating to the acceptance calculation, two additional simulations were also performed. These simulations calculated how much the total acceptance increases or decreases when the main acceptance parameters $\left(\delta, \theta_{t g t}, \phi_{t g t}\right)$ of the two HRS spectrometers are increased and decreased by twice the detector resolution. To increase or decrease the BigBite spectrometer acceptance, the horizontal angular acceptance was varied in width and also in location (more forward or backward in angle from the beam and target) by the average statistical error of the surveys of the centre location of the scintillator bars. The results are shown in table 4.2. The systematic errors for the acceptance are included in the cross section systematic error calculation by propagating through the higher and lower acceptance estimates.

\begin{tabular}{|c|c|c|c|}
\hline Kinematic Setting & CK1 & CK2 & CK3 \\
\hline \hline$N_{\text {sim }}$ & $667 \pm 25.83$ & $814 \pm 28.53$ & $719 \pm 26.81$ \\
\hline$N_{\text {sim-UA }}$ & $774 \pm 27.82$ & $892 \pm 29.87$ & $848 \pm 29.12$ \\
\hline$N_{\text {sim-LA }}$ & $533 \pm 23.52$ & $685 \pm 26.17$ & $580 \pm 24.08$ \\
\hline$A_{c}\left(\times 10^{-8} s r\right)$ & $8.12 \pm 0.31$ & $9.90 \pm 0.35$ & $8.75 \pm 0.33$ \\
\hline$A_{c-U A}\left(\times 10^{-8} s r\right)$ & $9.42 \pm 0.34$ & $10.85 \pm 0.36$ & $10.31 \pm 0.35$ \\
\hline$A_{c-L A}\left(\times 10^{-8} s r\right)$ & $6.49 \pm 0.29$ & $8.34 \pm 0.32$ & $7.06 \pm 0.29$ \\
\hline
\end{tabular}

Table 4.2: Acceptance calculations for the three kinematic settings. $N_{\text {sim }}$ are the number of simulated events that pass the acceptance of all three detectors out of 100 million generated events $\left(N_{\text {gen }}\right)$ for the three kinematic settings of CK1, CK2 and CK3. Acc is the the acceptance calculated using 4.1. $N_{s i m-U A}$ and $N_{s i m-L A}$ are the number of simulated events from the simulations of the upper $\left(A_{c-U A}\right)$ and lower $\left(A_{c-L A}\right)$ possible total acceptance.

\subsection{Luminosity}

The luminosity $L$ is defined as:

$$
L=\frac{\rho t N_{e} N_{A}}{A}
$$

where,

$A$ is the atomic mass of the ${ }^{12} \mathrm{C}=12.01 \mathrm{gmol}^{-1}$

$\rho$ is the density of ${ }^{12} \mathrm{C}=1.68 \mathrm{gcm}^{-3}$

$t$ is the thickness of the target along the beam $=0.0731 \mathrm{~cm}$

$N_{A}$ is Avogadro's number $=6.022 \times 10^{23} \mathrm{~mol}^{-1}$

$N_{e}$ is the number of incident electrons $=\frac{Q}{e}$, where $Q$ is the accumulated beam charge and $e$ is the charge of the electron. The accuracy of the Beam 
Charge Monitors (BCM) is $\leq 0.005$.

The luminosity was corrected to account for a number of other normalising factors, including the dead time and the efficiencies of the triggers used and the tracking and PID efficiencies of the spectrometers.

The probability of the production trigger firing due to the detection of a charged particle is represented by the trigger efficiency for each spectrometer. This also takes into account other factors such as the inefficiencies of the photomultiplier tubes on each scintillator bar, geometric holes between scintillator bars, fluctuations in energy deposited by charged particles in scintillators and the transmission efficiency of scintillation light along bars to the photomultiplier tubes. The trigger efficiency for electrons and protons is determined by the ratio of the number of primary event triggers to the total number of triggers. Across all the kinematic settings the efficiency of the proton and electron event triggers was determined to be better than $0.999 \pm 0.001$. For the BigBite spectrometer, the trigger efficiency is factored into the overall efficiency of BigBite also including PID and tracking efficiency.

The tracking efficiency for each HRS spectrometer is the probability for a charged particle to be observed and measured by the vertical drift chambers (VDC) and for the track to be reconstructed properly by the tracking software. The tracking efficiency was measured by creating a data sample of electrons or protons in the left or right HRS while not using any information obtained from the VDC of the particular HRS in question. This was achieved by using cuts on raw TDC values to form a coincidence time between both HRS's, particle identification cuts, trigger scintillator plane trigger cuts and acceptance cuts on the $\theta_{t g t}, \phi_{t g t}$ and $\delta_{t g t}$ variables of the opposing HRS. The tracking efficiency of each spectrometer was determined by taking the ratio of the number of events from the data sample that remain after the application of cuts based on VDC information to the number of events within the original data sample. The track reconstruction efficiency for each kinematic setting was determined to be $0.97 \pm 0.01$.

The PID efficiency of the left HRS was measured by determining the number of misidentified electrons that survive the main electron PID cut using the preshower and shower counters (see section 3.2.3). This was achieved by counting the number of electrons within the pre-shower shower pion cut region that are identified as electrons by the gas Cherenkov detector. The particle identification efficiency for the left HRS was found to be $0.997 \pm 0.001$. It was determined that no particle identification efficiency for the right HRS was required because after the initial proton selection using a cut on $\beta$, obtained from time of flight, the 
complete removal of any remaining pions and deuterons was achieved by the cut on the coincidence time of the left and right HRS. The signal to background for the events within the cut region of coincidence time of the scattered electron and proton in the left and right HRS was $97 \pm 0.5 \%$.

To measure the overall efficiency of BigBite, the probability of a proton being detected in BigBite was measured using the elastic reaction ${ }^{1} H\left(e, e^{\prime} p\right)$. The number of elastically scattered protons that fall within the acceptance of BigBite was measured and compared to the actual number of corresponding hits measured in BigBite. It was found that the detection efficiency for protons was $60 \pm 3.8 \%$. The main factor in BigBite detection efficiency is the detection of proton and deuteron hits in the thin auxiliary scintillator plane. The probability of detecting deuterons should be similar to that of protons, but maybe a little higher due to their heavier mass and slower velocities meaning slightly more energy should be deposited in the auxiliary plane. The overall efficiency of BigBite was therefore determined to be $60 \%$, the proton detection probability, but in reality it should be marginally higher for the ${ }^{12} C\left(e, e^{\prime} p d\right)$ reaction because BigBite is detecting deuterons.

When an event is detected by the spectrometer detectors there is a small finite time required for the data acquisition system (DAQ) to read out the data. This is called the deadtime because the DAQ cannot record any subsequent events during this time. These missing events however, are recorded in the number of events of each trigger type continuously counted by the scalers. This means the deadtime can be accounted for by comparing the number of events recorded in the data by the DAQ to total number of events of each trigger type registered on the scalers. The deadtime of the DAQ averaged out to $13.0 \pm 0.2 \%$, therefore the number of beam electrons needed to be scaled by a factor of $0.87 \pm 0.002$. Electronic deadtime also exists and it is the time needed by the electronics to process an event. At high event rates, information can be lost due to a pile up of signals. For this experiment, the highest event rate $(\leq 4 \mathrm{kHz})$ was small enough for the electronic deadtime to be neglected.

Systematic errors of the luminosity, tracking efficiency and of the other normalising factors multiplicative in the cross section of the ${ }^{12} C\left(e, e^{\prime} p\right)$ reaction discussed in this section has been calculated to be $1.6 \%$ by [9]. The systematic uncertainty with the overall efficiency of BigBite was determined to be $1.5 \%$. Therefore the total systematic uncertainty of the luminosity and the applied normalising corrections is $3.1 \%$. The results of the luminosity calculations and the associated statistical and systematic errors are presented in table 4.3. 


\begin{tabular}{|c|c|c|}
\hline Kinematic Setting & $Q$ & $L$ \\
\hline \hline CK1 & $22.443 \pm 0.121 C$ & $1.688 \pm 0.204 \pm 0.052 \times 10^{42} \mathrm{~cm}^{-2} \mathrm{~s}^{-1}$ \\
\hline CK2 & $20.158 \pm 0.101 C$ & $1.516 \pm 0.184 \pm 0.047 \times 10^{42} \mathrm{~cm}^{-2} \mathrm{~s}^{-1}$ \\
\hline CK3 & $14.377 \pm 0.072 C$ & $1.081 \pm 0.131 \pm 0.034 \times 10^{42} \mathrm{~cm}^{-2} s^{-1}$ \\
\hline
\end{tabular}

Table 4.3: The total charge measured by the beam charge monitors and the luminosites with statistical and systematic errors for each kinematic setting after the application of normalisation correction factors. 


\section{Chapter 5}

\section{Results and Conclusion}

The analysis presented in this thesis is the first measurement of differential cross section upper limit of the ${ }^{12} C\left(e, e^{\prime} p d\right)$ reaction at high energy transfer $(\omega=0.865$ $\mathrm{GeV})$. The kinematics corresponded to the quasi-free knockout of single protons with $|\vec{q}|=1.65 \mathrm{GeV} / \mathrm{c}, Q^{2}=2(G e V / c)^{2}$ and $x_{B}=\frac{Q^{2}}{2 M \omega}=1.2$. The differential cross section was calculated using the following equation:

$$
\frac{d \sigma}{d \Omega}=\frac{Y}{A_{c} L}
$$

where $Y$ is the experimental yield, $A_{c}$ is the acceptance and $L$ is the luminosity. The differential cross section results and errors are presented in table 5.1 and displayed in fig. 5.1.

\begin{tabular}{|c|c|}
\hline Kinematic Setting & $\frac{d \sigma}{d \Omega}$ \\
\hline \hline CK1 & $52.567 \pm 20.699+276.162-52.567 \mathrm{pb} / \mathrm{sr}$ \\
\hline CK2 & $191.354 \pm 43.087+114.780-73.567 \mathrm{pb} / \mathrm{sr}$ \\
\hline CK3 & $410.526 \pm 83.942+134.954-85.958 \mathrm{pb} / \mathrm{sr}$ \\
\hline
\end{tabular}

Table 5.1: Differential cross section results with statistical and asymmetric systematic errors respectively for the three kinematic settings corresponding to the missing momentum of the ${ }^{12} C\left(e, e^{\prime} p\right)$ reaction of $300(\mathrm{CK} 1), 400(\mathrm{CK} 2)$ and 500 (CK3) $M e V / c$. 


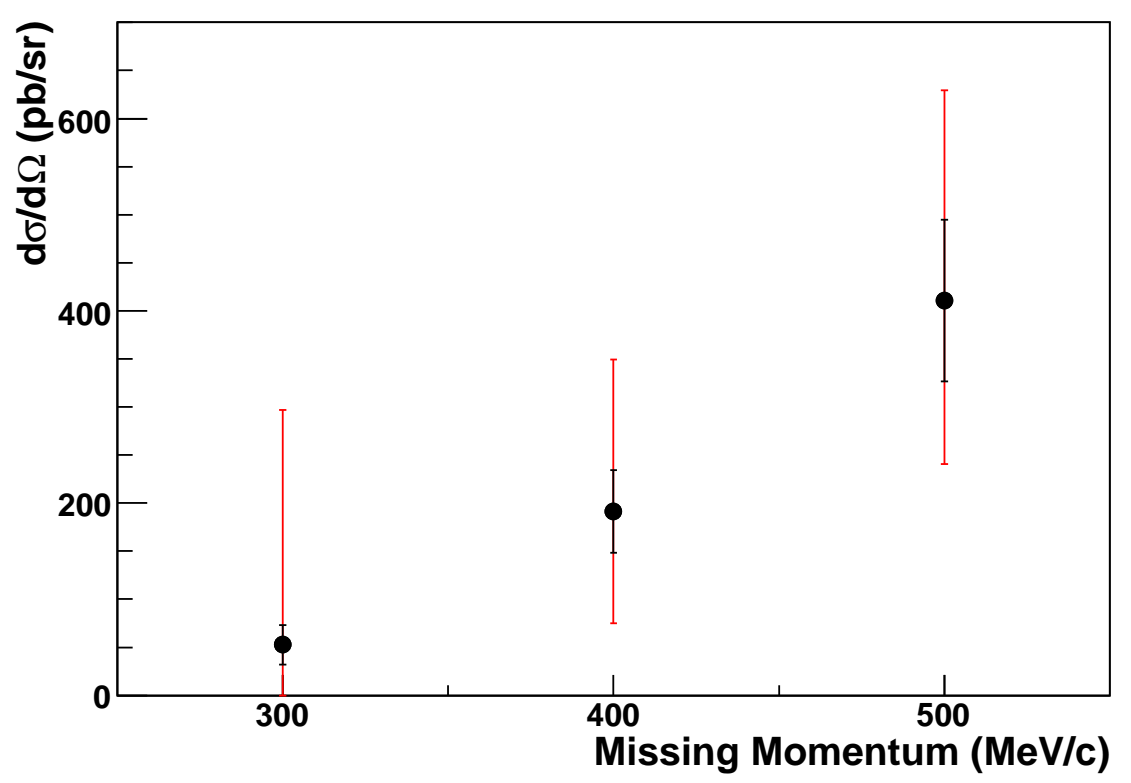

Figure 5.1: Differential cross section results with statistical (black error bars) and asymmetric systematic errors (red error bars) for the three kinematic settings corresponding to the missing momentum of the ${ }^{12} C\left(e, e^{\prime} p\right)$ reaction of 300 (CK1), $400(\mathrm{CK} 2)$ and $500(\mathrm{CK} 3) \mathrm{MeV} / \mathrm{c}$.

The fractional statistical errors of the yield, luminosity and acceptance were combined quadratically to produce the differential cross section statistical errors. The asymmetric systematic errors of the differential cross section were calculated by determining the difference between the nominal cross section values and the highest and lowest possible differential cross section values calculated when using equation 5.1 with the yield, acceptance and luminosity variables modified to include their systematic errors.

The differential cross sections for the ${ }^{12} C\left(e, e^{\prime} p d\right)$ reaction are very small and they increase as the missing momenta increases for the ${ }^{12} C\left(e, e^{\prime} p\right)$ reaction. There are few possible reaction mechanisms that could be contributing to the cross section, such as one body hadronic terms + the final state interaction (FSI) of the struck nucleon with a correlated NN pair, two body hadronic terms + a pickup FSI (where one of the nucleons captures an extra nucleon), two step $3 \mathrm{~N}$ processes and direct $3 \mathrm{~N}$ processes.

The experimental kinematics of this experiment were chosen to suppress two body processes such as meson exchange currents (MEC) or isobar currents (IC) to enhance the probability of measuring NN pairs correlated at short range (SRC). The high energy of this reaction, also reduces the contribution one of the nucleons 
from picking up another nucleon in a final state interaction for the two body processes or for the NN SRC processes for which the experiment was designed to favour.

The contribution of two-step $3 \mathrm{~N}$ and direct $3 \mathrm{~N}$ processes to the differential cross section are likely to be at short range and involve the exchange of heavy mesons such as the $\rho$ rather than $\pi \mathrm{s}$. In the ${ }^{3} H e(\gamma, p d)$ reaction measured at MAMI [5], the differential cross section is dominated by two body processes at forward proton centre of mass (c.m.) angles in the scattering plane up to $90^{\circ}$. At the backward c.m. angles of the proton there is a noticeable bump in the differential cross section that is attributed to $3 \mathrm{~N}$ processes. In the current experiment, the proton was measured at angles of $32.0^{\circ}$ to $40.1^{\circ}$, which is in the region where the reaction is thought to be dominated by $2 \mathrm{~N}$ processes, although this is a electron scattering experiment and the kinematics are very different. The likely suppression of all these different reaction mechanisms may be the reason why the measured differential cross section is very low.

It is not possible to rule out any of the possible reaction mechanisms or favour the contribution of one other another without further experimental exploration of the ${ }^{12} C\left(e, e^{\prime} p d\right)$ reaction at these kinematics with dedicated theoretical support. Due to the very low statistics and limited angular acceptance range of this measurement, it was not possible to produce a more complete analysis in line with the other experiments discussed in the introduction. For a full analysis of this reaction it would require a greater angular and momentum range in the experimental acceptance of the proton and deuteron and a long beam time to produce enough data. The result of this measurement could be used in event rate studies for the planning of similar experiments in the future.

The ${ }^{12} C(\gamma, p d)$ reaction measured at MAMI [53], with tagged photons in the energy range of 150 to $400 \mathrm{MeV}$ demonstrates the kind of analysis required to fully investigate the ${ }^{12} C(\gamma, p d)$ reaction. This experiment was able to show how the cross section varied with missing momentum, photon energy and recoil momentum across a wide angular range of the detected proton. By using comparable analysis of the ${ }^{12} \mathrm{C}(\gamma, p p),{ }^{12} \mathrm{C}(\gamma, p n),{ }^{3} \mathrm{He}(\gamma, p d)$ and ${ }^{3} \mathrm{He}(\gamma, p p) n$ reactions they were able to draw indications of which initial shells the nucleons were emitted from and see the similarities and differences between in the behaviour of the cross sections with photon energy. The ${ }^{3} \mathrm{He}(\gamma, p p) n$ cross section [6] exhibits a strong enhancement in the region of the $\Delta(1232)$ resonance which is not seen in either of the ${ }^{3} \mathrm{He}(\gamma, p d)$ or ${ }^{12} \mathrm{C}(\gamma, p d)$ reaction. This is thought to be due to the same isospin selection rule effects as in the ${ }^{3} \mathrm{He}(\gamma, p d)$ reaction [1]. A future 
measurement of the ${ }^{12} C\left(e, e^{\prime} p d\right)$ reaction in conjunction with the ${ }^{12} C\left(e, e^{\prime} p p\right)$ and ${ }^{12} C\left(e, e^{\prime} p n\right)$ reactions over wider angular and energy ranges would be able to investigate the different reaction mechanisms, these isospin selection rule effects and also the influence of the form factor of the deuteron in the final state.

The measurement of ${ }^{12} C\left(e, e^{\prime} p\right),{ }^{12} C\left(e, e^{\prime} p p\right)$ and ${ }^{12} C\left(e, e^{\prime} p n\right)$ reactions in this experiment deduced that $20 \%$ of the nucleons in ${ }^{12} C$ formed correlated pairs, with $90 \pm 10 \%$ of these consisting of p-n SRC pairs, $5 \pm 1.5 \%$ consisting of p-p SRC pairs and by isospin symmetry, $5 \pm 1.5 \%$ consisting of n-n SRC [81,82]. Recently Hagen, Papenbrock and others [83] have shown that the nucleon-nucleon interaction from chiral effective field theory at order next-to-next-to-next-to leading order binds nuclei within $0.4 \mathrm{MeV}$ per nucleon compared to data, when $3 \mathrm{~N}$ forces are included and to $0.5 \mathrm{MeV}$ if only $2 \mathrm{~N}$ interactions are used.

The measurement of polarised observables would also enhance the analysis of the ${ }^{12} C\left(e, e^{\prime} p d\right)$ reaction. By comparing the cross sections and the photon asymmetries of ${ }^{12} \mathrm{C}(\vec{\gamma}, p d)$ reaction measured at photon energies of 170 to $350 \mathrm{MeV}$ at MAMI [44] to ${ }^{12} C(\vec{\gamma}, p d),{ }^{12} C(\vec{\gamma}, p n),{ }^{12} C(\vec{\gamma}, p p)$ and ${ }^{3} H e(\vec{\gamma}, p d)$ reactions and also to Faddeev predictions it was possible to analyse the likely contribution of different reaction mechanisms to the cross section. At missing energies above 40 $\mathrm{MeV}$, the cross section is likely to have a large contribution from the two step $3 N$ processes, such as real pion production on one nucleon and re-absorption by a nucleon pair. Below missing energies of $40 \mathrm{MeV}$, direct $3 \mathrm{~N}$ processes, such as $2 \pi$ exchange between three nucleons, are more likely to contribute to the cross section.

However, the $A\left(e, e^{\prime} p d\right)$ reaction and other reactions in light nuclei that are sensitive to three nucleon forces are still not fully understood at intermediate energies. Experimental and theoretical effort should be focused to improve the quality and the description of data at these lower energies before making a proper effort to investigate the reaction mechanisms at higher energies and in heavier nuclei. 


\section{Bibliography}

[1] J. M. Laget. Three-Body Exchange Mechanisms in the ${ }^{3} H e(\gamma, p)^{2} H$ Reaction. Phys. Rev., C38:2993-2996, 1988.

[2] A.A Belyaev et al. JETP. Lett., 40:1275, 1984.

[3] R. Skibiński, J. Golak, H. Witała, W. Glöckle, A. Nogga, and H. Kamada. Polarization Observables in the Semiexclusive Photoinduced Three-Body Breakup of ${ }^{3}$ He. Phys. Rev. C, 72(4):044002, Oct 2005.

[4] D. Drechsel, O. Hanstein, S. S. Kamalov, and L. Tiator. A unitary isobar model for pion photo- and electroproduction on the proton up to $1-\mathrm{GeV}$. Nucl. Phys., A645:145-174, 1999.

[5] V. Isbert et al. Two-body photodisintegration of ${ }^{3} \mathrm{He}$ between $200-\mathrm{MeV}$ and 800-MeV. Nucl. Phys., A578:525-541, 1994.

[6] G. Audit et al. Study of three nucleon mechanisms in the photodisintegration of ${ }^{3}$ He. Nucl. Phys., A614:461-471, 1997.

[7] J.R.M Annand and D. Hamilton. Geant 4/C ++ Monte Carlo Studies of the BigBite Focal Plane Detector System, http://www.jlab.org/annand/BB.

[8] Application Software Group/ Geant. Detector description and simulation tool. Technical report, CERN, Geneva, Switzerland, 1993.

[9] P. Monaghan. Study of the ${ }^{12} C\left(e, e^{\prime} p\right)$ Reaction in a Correlations Dominant Regime with $Q^{2}=2$ and $X_{B}>1$. PhD thesis, Massachusetts Institute of Technology, 2008.

[10] J. Alcorn et al. Basic Instrumentation for Hall A at Jefferson Lab. Nucl. Instrum. Meth., A522:294-346, 2004.

[11] Hideki Yukawa. On the interaction of elementary particles. Proc. Phys. Math. Soc. Jap., 17:48-57, 1935. 
[12] L. Lapikás. Quasi-elastic electron scattering off nuclei. Nuclear Physics A, 553:297 - 308, 1993.

[13] R. Machleidt. The Meson theory of nuclear forces and nuclear structure. Adv. Nucl. Phys., 19:189-376, 1989.

[14] A. Nogga, H. Kamada, and W. Glöckle. Modern nuclear force predictions for the $\alpha$ particle. Phys. Rev. Lett., 85(5):944-947, Jul 2000.

[15] A. Kievsky M. Viviani and S. Rosati. Nuovo Cimento Soc. Ital. Fis A631, 111c, A631:111c, 1992.

[16] A. Nogga, H. Kamada, W. Glöckle, and B. R. Barrett. The $\alpha$ particle based on modern nuclear forces. Phys. Rev. C, 65(5):054003, May 2002.

[17] Steven C. Pieper, V. R. Pandharipande, R. B. Wiringa, and J. Carlson. Realistic models of pion-exchange three-nucleon interactions. Phys. Rev. C, 64(1):014001, Jun 2001.

[18] J. L. Friar, D. Hüber, and U. van Kolck. Chiral symmetry and three-nucleon forces. Phys. Rev. C, 59(1):53-58, Jan 1999.

[19] B. S. Pudliner, V. R. Pandharipande, J. Carlson, Steven C. Pieper, and R. B. Wiringa. Quantum Monte Carlo Calculations of Nuclei with $A<7$. Phys. Rev. C, 56(4):1720-1750, Oct 1997.

[20] Novel Three-Nucleon-Force Terms in the Three-Nucleon System", journal = "Few-Body Systems. 30(1):95 - 120, 2001.

[21] J. Carlson, V. R. Pandharipande, and R. B. Wiringa. Three-nucleon interaction in 3-, 4- and $\infty$-body systems. Nuclear Physics A, 401(1):59 - 85, 1983.

[22] S. A. Coon, M. D. Scadron, P. C. McNamee, B. R. Barrett, D. W. E. Blatt, and B. H. J. McKellar. The two-pion-exchange three-nucleon potential and nuclear matter. Nuclear Physics A, 317(1):242 - 278, 1979.

[23] Jun ichi Fujita and Hironari Miyazawa. Pion theory of three-body forces. Progress of Theoretical Physics, 17(3):360-365, 1957.

[24] E. Epelbaum et al. Few nucleon systems with two-nucleon forces from chiral effective field theory. Eur. Phys. J., A15:543-563, 2002. 
[25] E. Epelbaum, A. Nogga, W. Glöckle, H. Kamada, Ulf-G. Meissner, and H. Witala. Three-nucleon Forces from Chiral Effective Field Theory. Phys. Rev. C, 66(6):064001, Dec 2002.

[26] E. Epelbaum, H. Krebs, and Ulf-G. Meissner. $\Delta$-excitations and the threenucleon force. Nuclear Physics A, 806(1-4):65 - 78, 2008.

[27] Steven Weinberg. Nuclear Forces from Chiral Lagrangians. Physics Letters $B, 251(2): 288-292,1990$.

[28] Steven Weinberg. Effective Chiral Lagrangians for Nucleon-Pion Interactions and Nuclear Forces. Nuclear Physics B, 363(1):3 - 18, 1991.

[29] U. van Kolck. Few-Nucleon Forces from Chiral Lagrangians. Phys. Rev. C, 49(6):2932-2941, Jun 1994.

[30] Shin Nan Yang and W. Glöckle. Three-Body Mesonic Retardation Effect. Phys. Rev. C, 33(5):1774-1778, May 1986.

[31] J. Gasser and A. Zepeda. Approaching the chiral limit in QCD. Nuclear Physics B, 174(2-3):445 - 473, 1980.

[32] J. A. Eden and M. F. Gari. Does the 3N force have a hard core? Phys. Rev. C, 53(4):1510-1518, Apr 1996.

[33] H. Witała, W. Glöckle, D. Hüber, J. Golak, and H. Kamada. Cross Section Minima in Elastic $N d$ Scattering: Possible Evidence for Three-Nucleon Force Effects. Phys. Rev. Lett., 81(6):1183-1186, Aug 1998.

[34] H. Sakai. Experimental status of three-nucleon force study by nucleondeuteron system. Nuclear Physics A, 790(1-4):122c - 128c, 2007. Few-Body Problems in Physics - FB 18, 18th International IUPAP Conference on FewBody Problems in Physics.

[35] K. S. Egiyan, N. B. Dashyan, M. M. Sargsian, M. I. Strikman, L. B. Weinstein, G. Adams, P. Ambrozewicz, M. Anghinolfi, B. Asavapibhop, G. Asryan, H. Avakian, H. Baghdasaryan, N. Baillie, J. P. Ball, N. A. Baltzell, V. Batourine, M. Battaglieri, I. Bedlinskiy, M. Bektasoglu, M. Bellis, N. Benmouna, A. S. Biselli, B. E. Bonner, S. Bouchigny, S. Boiarinov, R. Bradford, and D. Branford. Measurement of Two- and Three-Nucleon Short-Range Correlation Probabilities in Nuclei. Phys. Rev. Lett., 96(8):082501, Mar 2006. 
[36] L. L. Frankfurt and M. I. Strikman. High-energy phenomena, short-range nuclear structure and QCD. Physics Reports, 76(4):215 - 347, 1981.

[37] Leonid Frankfurt and Mark Strikman. Hard nuclear processes and microscopic nuclear structure. Physics Reports, 160(5-6):235 - 427, 1988.

[38] J. M. Laget. Three-Body Exchange Currents. 1. The ${ }^{3} H e(\gamma, 2 p) n$ Reaction. J. Phys., G14:1445-1451, 1988.

[39] R. G. Arnold, D. Benton, P. Bosted, L. Clogher, G. DeChambrier, A. T. Katramatou, J. Lambert, A. Lung, G. G. Petratos, A. Rahbar, S. E. Rock, Z. M. Szalata, B. Debebe, M. Frodyma, R. S. Hicks, A. Hotta, G. A. Peterson, R. A. Gearhart, J. Alster, J. Lichtenstadt, F. Dietrich, and K. van Bibber. Measurements of transverse quasielastic electron scattering from the deuteron at high momentum transfers. Phys. Rev. Lett., 61(7):806-809, Aug 1988.

[40] D. B. Day, J. S. McCarthy, Z. E. Meziani, R. Minehart, R. Sealock, S. T. Thornton, J. Jourdan, I. Sick, B. W. Filippone, R. D. McKeown, R. G. Milner, D. H. Potterveld, and Z. Szalata. y scaling in electron-nucleus scattering. Phys. Rev. Lett., 59(4):427-430, Jul 1987.

[41] B. A. Mecking et al. The CEBAF large acceptance spectrometer (CLAS). Nucl. Instrum. Methods Phys. Res A, 503(3):513 - 553, 2003.

[42] L.C. Maximon and F. Prats. Proc. 10th int. conf. on particle and nuclei heidelberg. 1984.

[43] F. Prats. Phys. Lett. B, 88:23, 1979.

[44] D. P. Watts, J. Ahrens, J. R. M. Annand, R. Beck, D. Branford, P. Grabmayr, T. Hehl, J. D. Kellie, I. J. D. MacGregor, J. C. McGeorge, and R. O. Owens. Three-nucleon mechanisms in photoreactions. Physics Letters B, 553(1-2):25 - 30, 2003.

[45] R. C. Carrasco and E. Oset. Interaction of real photons with nuclei from 100 to $500 \mathrm{MeV}$. Nuclear Physics A, 536(3-4):445 - 508, 1992.

[46] R. C. Carrasco, M. J. Vicente Vacas, and E. Oset. Inclusive $(\gamma, N),(\gamma, N N)$ and $(\gamma, N \pi)$ Reactions in Nuclei at Intermediate Energies. Nuclear Physics A, 570(3-4):701 - 721, 1994. 
[47] R. C. Carrasco, E. Oset, and L. L. Salcedo. Inclusive $(\gamma, \pi)$ reactions in nuclei. Nuclear Physics A, 541(4):585 - 622, 1992.

[48] B. Kotlinski et al. Initial State Interaction in the $(\pi+, 3 \mathrm{p})$ Reaction on $\mathrm{N}$, Ar and Xe. Eur. Phys. J. A., 1:435 - 445, 1998.

[49] D. P. Watts, I. J. D. MacGregor, J. Ahrens, J. R. M. Annand, R. Beck, D. Branford, P. Grabmayr, S. J. Hall, P. D. Harty, T. Hehl, J. D. Kellie, T. Lamparter, M. Liang, J. A. MacKenzie, S. J. McAllister, J. C. McGeorge, R. O. Owens, M. Sauer, R. Schneider, G. J. Wagner, and T. T-H. Yau. The ${ }^{12} c(\gamma, n n)$ reaction studied over a wide kinematic range. Phys. Rev. C, 62(1):014616, Jun 2000.

[50] G. E. Cross, I. J. D. MacGregor, J. C. McGeorge, J. Ahrens, I. Anthony, J. R. M. Annand, R. Beck, D. Branford, T. Davinson, P. Grabmayr, S. J. Hall, P. D. Harty, T. Hehl, J. D. Kellie, T. Lamparter, L. Machenil, J. A. MacKenzie, G. J. Miller, R. O. Owens, J. Ryckebusch, M. Sauer, R. Schneider, K. Spaeth, and M. Vanderhaeghen. Proton photoproduction from ${ }^{12} C$. Nuclear Physics A, 593(4):463 - 487, 1995.

[51] D.P. Watts, J.R.M. Annand, R. Beck, D. Branford, D.I. Glazier, P. Grabmayr, K. Livingston, I.J.D. MacGregor, J.C. McGeorge, R.O. Owens, and G. Rosner. Dependence of the ${ }^{12} C(\vec{\gamma}, p d)$ Reaction on Photon Linear Polarisation. Physics Letters B, 647(2-3):88 - 92, 2007.

[52] J. Robinson. Ph.D. thesis. PhD thesis, University of Glasgow, 2010.

[53] S. J. McAllister, J. C. McGeorge, I. J. D. MacGregor, J. R. M. Annand, S. J. Hall, P. D. Harty, J. D. Kellie, G. J. Miller, R. O. Owens, D. P. Watts, T. TH. Yau, D. Branford, J. A. MacKenzie, M. Liang, P. Grabmayr, T. Hehl, T. Lamparter, M. Sauer, R. Schneider, J. Ahrens, and R. Beck. The $(\gamma, p d)$ reaction in ${ }^{12}$ C. Phys. Rev. C, 60(4):044610, Sep 1999.

[54] J. C. McGeorge, I. J. D. MacGregor, S. N. Dancer, J. R. M. Annand, I. Anthony, G. I. Crawford, S. J. Hall, P. D. Harty, J. D. Kellie, G. J. Miller, R. O. Owens, P. A. Wallace, D. Branford, A. C. Shotter, B. Schoch, R. Beck, H. Schmieden, and J. M. Vogt. $(\gamma, 2 N)$ reaction in ${ }^{12} C$. Phys. Rev. C, 51(4):1967-1976, Apr 1995.

[55] P. D. Harty, I. J. D. MacGregor, P. Grabmayr, J. Ahrens, J. R. M. Annand, I. Anthony, R. Beck, D. Branford, G. E. Cross, T. Davinson, S. J. Hall, 
T. Hehl, J. D. Kellie, T. Lamparter, J. A. MacKenzie, J. C. McGeorge, G. J. Miller, R. O. Owens, M. Sauer, R. Schneider, and K. Spaeth. The Contribution of $2 \mathrm{~N}$ Photon Absorption in ${ }^{12} C(\gamma, 2 N)$ Reactions for $E_{\gamma}=$ 150-400 MeV. Physics Letters B, 380(3-4):247 - 252, 1996.

[56] J. R. M. Annand I. Anthony R. Beck D. Branford G. E. Cross T. Davinson P. Grabmayr S. J. Hall P. D. Harty T. Hehl J. D. Kellie I. J. D. MacGregor J. A. MacKenzie J. C. McGeorge G. J. Miller R. O. Owens M. Sauer R. Schneider K. Spaeth G. J. Wagner Th. Lamparter, J. Ahrens. On photonuclear reaction mechanisms at intermediate energies. Z. Phys. A, 355:1 - 3, 1996.

[57] S. J. McAllister. Ph.D. thesis. PhD thesis, University of Glasgow, 1997.

[58] L. R. B. Elton and A. Swift. Single-particle potentials and wave functions in the 1p and 2s-1d shells. Nuclear Physics A, 94(1):52 - 72, 1967.

[59] D. G. Middleton, J. R. M. Annand, M. Ases Antelo, C. Ayerbe, P. Barneo, D. Baumann, J. Bermuth, J. Bernauer, H. P. Blok, R. Böhm, D. Bosnar, M. Ding, M. O. Distler, J. Friedrich, J. García Llongo, D. I. Glazier, J. Golak, W. Glöckle, P. Grabmayr, T. Hehl, J. Heim, W. H. A. Hesselink, E. Jans, H. Kamada, G. Jover Mañas, M. Kohl, and L. Lapikás. Investigation of the Exclusive ${ }^{3} \mathrm{He}\left(e, e^{\prime} p n\right)^{1} \mathrm{H}$ Reaction. Phys. Rev. Lett., 103(15):152501, Oct 2009 .

[60] D. L. Groep, M. F. van Batenburg, Th. S. Bauer, H. P. Blok, D. J. Boersma, E. Cisbani, R. De Leo, S. Frullani, F. Garibaldi, W. Glöckle, J. Golak, P. Heimberg, W. H. A. Hesselink, M. Iodice, D. G. Ireland, E. Jans, H. Kamada, L. Lapikás, G. J. Lolos, R. Perrino, A. Scott, R. Starink, M. F. M. Steenbakkers, G. M. Urciuoli, H. de Vries, L. B. Weinstein, and H. Witała. Investigation of the Exclusive ${ }^{3} \mathrm{He}\left(e, e^{\prime} p p\right) n$ Reaction. Phys. Rev. Lett., 83(26):5443-5446, Dec 1999.

[61] D. L. Groep, M. F. van Batenburg, Th. S. Bauer, H. P. Blok, D. J. Boersma, E. Cisbani, R. De Leo, S. Frullani, F. Garibaldi, W. Glöckle, J. Golak, P. Heimberg, W. H. A. Hesselink, M. Iodice, D. G. Ireland, E. Jans, H. Kamada, L. Lapikás, G. J. Lolos, C. J. G. Onderwater, R. Perrino, A. Scott, R. Starink, M. F. M. Steenbakkers, G. M. Urciuoli, H. de Vries, and L. B. Weinstein. Investigation of the exclusive ${ }^{3} \mathrm{He}\left(e, e^{\prime} p p\right) n$ reaction. Phys. Rev. C, 63(1):014005, Dec 2000. 
[62] R. A. Niyazov, L. B. Weinstein, G. Adams, P. Ambrozewicz, E. Anciant, M. Anghinolfi, B. Asavapibhop, G. Asryan, G. Audit, T. Auger, H. Avakian, H. Bagdasaryan, J. P. Ball, S. Barrow, M. Battaglieri, K. Beard, M. Bektasoglu, M. Bellis, N. Benmouna, B. L. Berman, W. Bertozzi, N. Bianchi, A. S. Biselli, S. Boiarinov, B. E. Bonner, S. Bouchigny, and R. Bradford. Two-Nucleon Momentum Distributions Measured in ${ }^{3} H e\left(e, e^{\prime} p p\right) n$. Phys. Rev. Lett., 92(5):052303, Feb 2004.

[63] E. van Meijgaard and J. A. Tjon. Three-body electrodisintegration of the trinucleon system. Phys. Rev. C, 45(4):1463-1486, Apr 1992.

[64] J. Golak, R. Skibinski, H. Witala, W. Glockle, A. Nogga, and H. Kamada. Electron and photon scattering on three-nucleon bound states. Physics Reports, 415(2-3):89 - 205, 2005.

[65] A. Deltuva, L. P. Yuan, J. Adam, and P. U. Sauer. Three-body electrodisintegration of the three-nucleon bound state with $\Delta$-isobar excitation: Processes below pion-production threshold. Phys. Rev. C, 70(3):034004, Sep 2004.

[66] J. Watson W. Bertozzi, E. Piasetzky and S. Wood. Jefferson Lab Hall A Proposal (2000).

[67] R. Subedi. Studying Short-Range Corellations with the ${ }^{12} C\left(e, e^{\prime} p n\right)$ Reaction. $\mathrm{PhD}$ thesis, Kent State University, 2007.

[68] R. Shneor. Investigation of Proton-Proton Short-Range Corellations via the ${ }^{12} C\left(e, e^{\prime} p p\right)$ Reaction. PhD thesis, Tel Aviv University, 2008.

[69] C. W. Leemann, D. R. Douglas, and G. A. Krafft. The Continuous Electron Beam Accelerator Facility: CEBAF at the Jefferson Laboratory. Ann. Rev. Nucl. Part. Sci., 51:413-450, 2001.

[70] The Hall A cryotarget system website, http://www.hallaweb.jlab.org/equipment/targts/cryotargets/.

[71] M. Modice et al. Nucl. Instr. Meth., A411:223, 1998.

[72] D. J. J. de Lange et al. A large acceptance spectrometer for the internal target facility at NIKHEF. Nucl. Instrum. Meth., A406:182-194, 1998. 
[73] D. J. J. de Lange et al. The optical properties of the BigBite spectrometer at NIKHEF. Nucl. Instrum. Meth., A412:254-264, 1998.

[74] Technical report Jefferson Lab online data acquisition. http://coda.jlab.org.

[75] Hall A C ++ Analyzer, http://hallaweb.jlab.org/root/index.html/.

[76] Rene Brun. Root. an object-oriented data analysis framework, http://root.cern.ch.

[77] K. G. Fissum, W. Bertozzi, J. P. Chen, D. Dale, H. C. Fenker, J. Gao, A. Gavalya, S. Gilad, C. R. Leathers, N. Liyanage, R. O. Michaels, E. A. J. M. Offermann, J. Segal, J. A. Templon, R. Wechsler, B. Wojtsekhowski, and J. Zhao. Vertical drift chambers for the Hall A high-resolution spectrometers at Jefferson Lab. Nuclear Instruments and Methods in Physics Research Section A: Accelerators, Spectrometers, Detectors and Associated Equipment, 474(2):108 - 131, 2001.

[78] N. Liyanage. Optics calibration of the hall a high resolution spectrometers. JLab Technical Note, TN 02-012.

[79] Particle data book, chapter 27 - passage of particles through matter, http://pdg.lbl.gov/.

[80] R. M. Sternheimer, S. M. Seltzer, and M. J. Berger. Density effect for the ionization loss of charged particles in various substances. Phys. Rev. B, 26(11):6067-6076, Dec 1982.

[81] R. Subedi et al. Probing cold dense nuclear matter. Science, 320 no. 5882:1476-1478, 2008.

[82] R. Shneor et al. Investigation of Proton-Proton Short-Range Correlations via the ${ }^{12} C\left(e, e^{\prime} p p\right)$ Reaction. Phys. Rev. Lett., 99:072501, 2007.

[83] G. Hagen, T. Papenbrock, D. J. Dean, and M. Hjorth-Jensen. Ab initio coupled-cluster approach to nuclear structure with modern nucleon-nucleon interactions. Phys. Rev. C, 82(3):034330, Sep 2010. 